UNIVERSIDADE DE SÃO PAULO

INSTITUTO DE FÍSICA DE SÃO CARLOS

NORBERTO HELIL PASQUA

\title{
TEORIA ENTRÓPICA DA NUCLEAÇÃO E FUNÇÃO ENTROPIA APLICADAS À CONDENSAÇÃO DO VAPOR D’ÁGUA
}

SÃO CARLOS

2007 


\section{NORBERTO HELIL PASQUA}

\section{TEORIA ENTRÓPICA DA NUCLEAÇÃO E FUNÇÃO ENTROPIA APLICADAS À CONDENSAÇÃO DO VAPOR D’ÁGUA}

Tese apresentada ao Instituto de Física de São Carlos para obtenção do título de doutor em Física Básica

Área de concentração: Física Básica Orientador: Prof. Dr. Bernhard Joachim Mokross 
AUTORIZO A REPRODUÇÃO E DIVULGAÇÃO TOTAL OU PARCIAL DESTE TRABALHO, POR QUALQUER MEIO CONVENCIONAL OU ELETRÔNICO, PARA FINS DE ESTUDO E PESQUISA, DESDE QUE CITADA A FONTE.

Ficha catalográfica elaborada pelo

Departamento Técnico do Sistema Integrado de Bibliotecas da USP

Pasqua, Norberto Helil.

Teoria Entrópica da Nucleação e Função Entropia Aplicadas à Condensação do Vapor d'Água / Norberto Helil Pasqua; orientador Bernhard Joachim Mokross.

-- São Carlos, 2007.

$124 \mathrm{p}$.

Tese (Doutorado - Programa de Pós-Graduação em Física. Área de concentração: Física Básica) — Instituto de Física de São Carlos, Universidade de São Paulo.

1. Nucleação. 2. Teoria Entrópica da Nucleação. 3. Função Entropia Metaestável. 4. Flutuação. 5. Água. I. Título. 


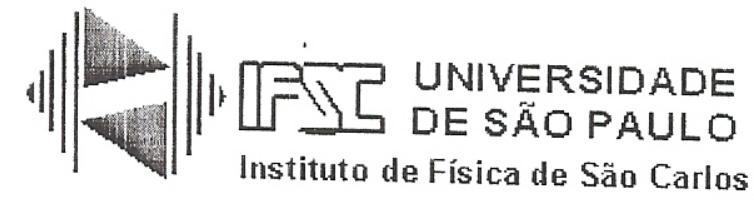
Caima Postal 269
$13500-970$ Sa Carlos, SP
An. Trabalhador São-carlense 400 , 13566-590 Sá Canos, SP
Fone'Fax (16) $3373-5777$
WWW. if sc. Lsp br br
swposg ado ifscusp.br

MEMBROS DA COMISSÃO JULGADORA DA TESE DE DOUTORADO DE NORBERTO HELIL PASQUA 24/08/2007.

\section{COMISSÃO JULGADORA:}

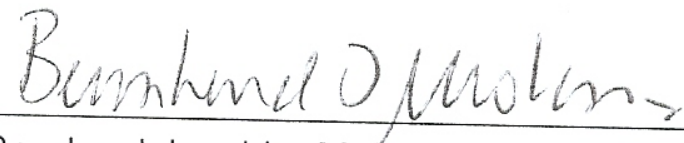

Prof(a). Dr(a). Bernhard Joachim Mokross (Orientador e Presidente) - IFSC/USP

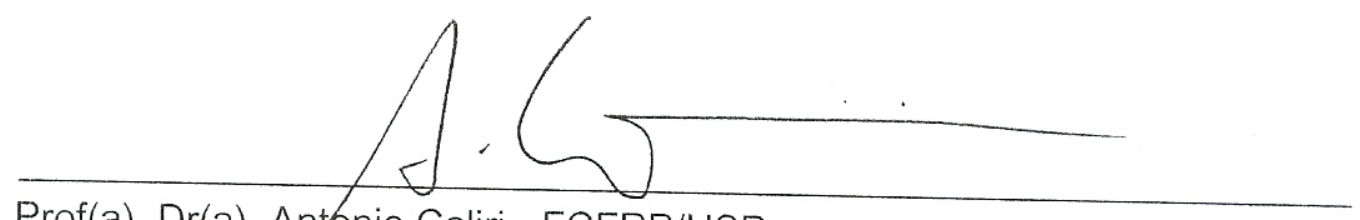

Prof(a). Dr(a). Antonio Caliri - FCFRP/USP

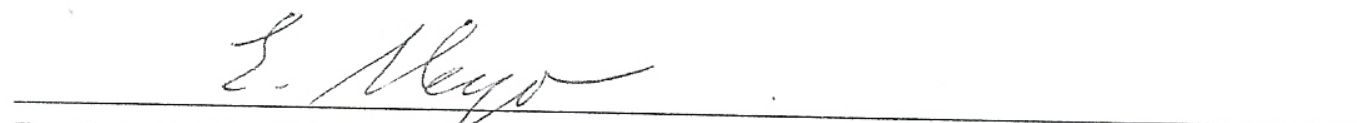

Prof(a). Dr(a). Erich Meyer - UFRJ
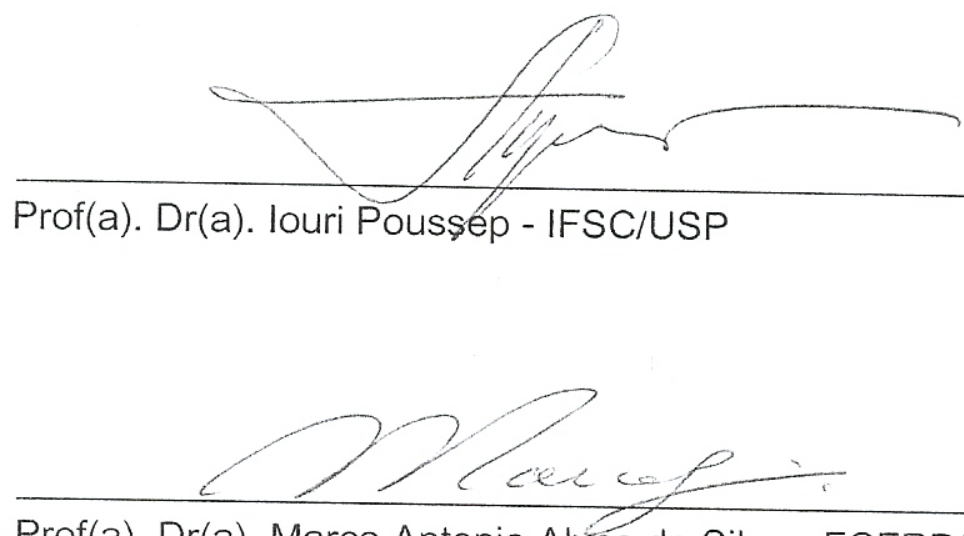

Prof(a). Dr(a). Marco Antonio Alves da Silva - FCFRP/USP 


\section{Dedicatória}

Ao meu pai,

ao meu filho,

à minha Parê. 


\section{Agradecimentos}

Ao mestre Prof. Dr. Bernhard Joachim Mokross, por toda força desprendida também no além livros.

Ao Instituto de Física de São Carlos, pelo apoio institucional e pelas facilidades oferecidas.

Ao CNPq, pelo apoio financeiro.

Aos funcionários da Secretaria de Pós-Graduação e da Biblioteca que sempre se dispuseram prontamente a auxiliar na superação dos percalços.

Aos professores Dr. Esmerindo de Sousa Bernardes e Dr. Francisco Castilho Alcaraz, pela disposição em sanar minhas dúvidas.

Agradeço ao meu pai, Norberto Pasqua, pela sua existência e apoio incondicional, que muito ajudou em minha caminhada; à Parê minha mulher, amiga e companheira que esteve ao meu lado em todas as manifestações entrópicas do meu ser; e ao Igor, moleque presente.

Enfim, a todos que de alguma forma colaboraram para a realização deste trabalho, os meus mais sinceros agradecimentos. Faço menção àqueles meus familiares que acreditaram em mim. Agradeço ao João pelo apoio em diminuir minha entropia. Agradeço aos meus amigos João Teles Carvalho Jr., Guilherme da Costa Pereira Innocentini, Gilberto Medeiros Nakamura, Alexandre Ferreira Ramos, pelas conversas e cumplicidades tão importantes para o nosso crescimento. 


\section{Prefácio}

O pano de fundo deste trabalho é o fenômeno da nucleação. É estudado a partir da Teoria Entrópica da Nucleação e aplicada à substância pura água. Desenvolveu-se um algoritmo para obter uma função entropia, a qual tem o papel de facilitar a análise do processo da forma mais acurada possível.

Os quatro primeiros capítulos fornecem uma visão geral da termodinâmica aplicada ao fenômeno da nucleação. Destacam-se processos isobáricos irreversíveis nos quais têm naturalmente as variáveis entropia e entalpia. A função entropia tem como variáveis a entalpia e a pressão.

No quinto capítulo é apresentada a Teoria Entrópica da Nucleação. Com a ajuda do diagrama de Mollier modificado para evidenciar a região metaestável de um fluido puro submetido a um processo isobárico, é obtida uma expressão para o cálculo do núcleo crítico, a partir da teoria de flutuação de Landau, a qual é revisitada no Apêndice E. Os cálculos preliminares com a equação de estado de van der Waals e depois com a de Peng-Robinson resultaram ser qualitativamente promissores. Porém é mostrado que a equação do núcleo crítico é incompleta, o que justifica o desenvolvimento no Capítulo 7 de uma abordagem do fenômeno em um ensemble a pressão constante, baseada na teoria de flutuação de Einstein. Além disso, as equações de estado do tipo van der Waals não conseguem representar suficientemente bem a entropia do sistema, conforme mostrado no Apêndice A. Finalmente o Apêndice F trata dos experimentos de nucleação com a água.

A necessidade do desenvolvimento de uma função entropia é evidenciada no Apêndice B. Assim, uma expressão da entropia em função da entalpia a pressão constante é desenvolvida no Capítulo 6, onde é mostrado que aspectos físicos e geométricos foram fundamentais. Aspectos algébricos do algoritmo são detalhados nos Apêndices C e D. 
Finalmente, os dados experimentais usados para comparar os resultados teóricos obtidos ao longo dos Capítulos 5 e 6, têm sua origem explicada no Apêndice F. 


\section{Resumo}

O fenômeno da nucleação é um processo intrinsecamente irreversível. A Teoria Entrópica da Nucleação (TEN) aborda-o analisando um processo reversível equivalente no qual há liberação de calor latente (variação da entalpia), concomitante a um rearranjo estrutural descrito pela variação da entropia antes e depois de certa quantidade de material ter nucleado. Para visualizar a dinâmica e facilitar a análise foi escolhido um processo isobárico.

O diagrama de Mollier modificado para evidenciar a região metaestável ajudou a desenvolver uma expressão para o cálculo do tamanho do núcleo crítico, mediante a teoria da flutuação de Landau. Para analisar o sistema na região metaestável, obteve-se a função entropia, $S\left(H, P_{0}\right)$, em que aspectos físicos e geométricos (como o princípio de estabilidade termodinâmica) foram determinantes. Cálculos do núcleo crítico em relação à temperatura mostraram concordância qualitativa com o trabalho de Dillmann-Meier. Porém, entende-se que a função do núcleo crítico está incompleta.

Para lidar com aglomerados e núcleos em uma abordagem termodinâmica, um ensemble a pressão constante é o mais apropriado, cuja variável conjugada é o volume. Com base em uma teoria das flutuações isotérmicas em um fluido ideal (Koper-Reiss), desenvolveu-se a teoria das flutuações não-isotérmicas (Mokross), aplicável a um fluido não-ideal metaestável mantido a pressão e temperatura constantes. Os parâmetros termodinâmicos do elemento de volume que flutua mudam e diferem daqueles do banho, e evolvem acordando com a equação de estado $S=S(H, P)$

Palavras chave: Nucleação. Teoria Entrópica da Nucleação. Função Entropia Metaestável. Flutuação. Água. 


\section{Abstract}

The phenomenon of nucleation is an intrinsically irreversible process. The nucleation is explored by the Entropic Nucleation Theory (ENT), in which, the irreversible process is replaced by an equivalent one, although now, the process is reversible in which there is a change in the enthalpy, and also an structural rearrangement coded in the change of de entropy. To study the dynamics and perform the analysis an isobaric process was chosen.

The metastable region was used to develop an expression for the calculation of size of the critical nucleus, having in mind the Landau's fluctuation theory. This region was obtained with the help of the modified Mollier diagram. The physical and geometric features of the system were crucial to obtain the entropy function, $S\left(H, P_{0}\right)$, used to analyze the metastable region. Calculations of the critical nucleus, with respect to the temperature, were in qualitative agreement with Dillmann-Meier work. Although, the function for the critical nucleus is incomplete.

To handle with clusters and nucleus in a thermodynamic framework, a constant pressure assemble is preferable having the volume as the conjugated variable. With the help of the isothermal fluctuation theory, in an ideal fluid (Koper-Reiss), the non-isothermal fluctuation theory (Mokross) was developed, and used to study a non-ideal metastable fluid kept at constant pressure and temperature. The thermodynamics parameters of the fluctuating volume element change, differing from those of the bath, and the state equation, $S=S(H, P)$ gives its evolution.

Keywords: Nucleation. Entropic Nucleation Theory. Metastable Entropy Function. Fluctuation. Water. 


\section{Lista de Figuras}

2.1 O tipo de interação entre sistema e vizinhança é importante para a descrição do

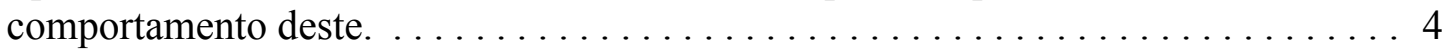

2.2 A produção de entropia é observada quando há gradiente de temperatura $T_{0}>T_{1}$

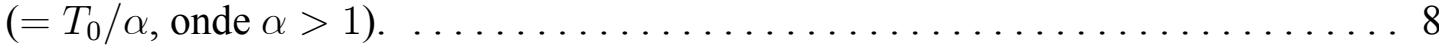

2.3 A linha laranja contínua representa o líquido e o vapor saturado (binodal); a pontilhada define o limite de estabilidade para fase líquida e gasosa (espinodal).

3.4 Diagrama de fases experimental da água pura, no qual se encontra evidenciada a curva pressão-vapor: coexistência das fases líquida e vapor. . . . . . . . . . . . . 16

4.5 O pico é caracterizado pelo equilíbrio entre as energia de volume e de superfície:

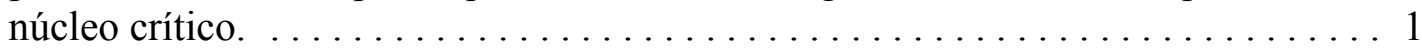

4.6 À esquerda a teoria de Dillmann-Meier encontra-se em melhor concordância com a experiência; à direita a estranha dependência da TCN com a temperatura. A

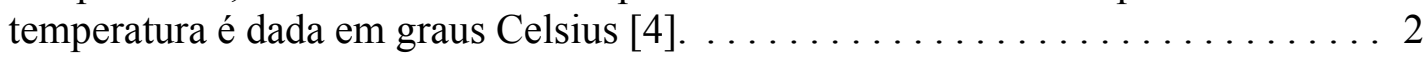

4.7 O trabalho de RKK possibilitou a consistência interna da TCN, porém mesmo com o fator 6000 ad hoc, a dependência estranha com a temperatura persiste. No

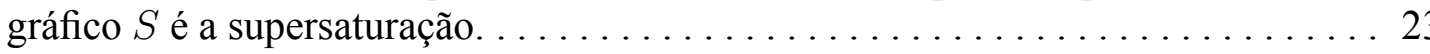

5.8 O diagrama de Mollier foi modificado para tornar a entropia função da entalpia e também para evidenciar a região da metaestabilidade. $\Delta h_{v a p}$ e $\Delta s_{v a p}$ são o calor e a entropia de vaporização, respectivamente. . . . . . . . . . . . . . . 27

5.9 A conexão entre as fases líquida e gasosa, devido à continuidade de estados, dá-se por uma curva suave que apresenta dois pontos de inflexão.

5.10 Flutuação da temperatura e da entalpia da fase gasosa (direita) e flutuação da

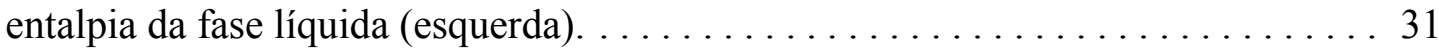

5.11 No diagrama de Mollier, o ponto $g m$ é o ponto do vapor no estado metaestável. . . . 36

5.12 Os pontos binodais, espinodais e metaestável estão sobre a reta $P=P_{e} \ldots \ldots . .37$

5.13 Núcleo crítico em função da temperatura. À esquerda a experiência de Viisanen et al. e à direita a experiência de Wölk-Strey. Em ambas, em preto é o núcleo crítico calculado pelo Método dos Mínimos Quadrados, em vermelho, com auxílio da EE-vdW, e em azul com ajuda da EE-PR.

6.14 Diagrama de Mollier da água. 
6.15 Diagrama de Mollier estilizado, em que se evidenciam as fases de um fluido puro

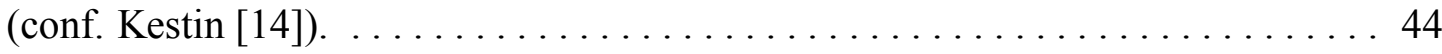

6.16 Os subíndices $l l, l e, l m, l s, g s, g m, g e, g g$ representam os pontos do líquido nos estados estável, ETD, metaestável, espinodal e do gás nos estados espinodal, metaestável, ETD, estável, respectivamente.

6.17 O cálculo de $T=\frac{\Delta h}{\Delta s}$ (em vermelho) e de $T=\frac{P \Delta v}{R}$ (em azul) com pontos experimentais [14], mostra que no ponto triplo $T_{3}=\frac{\Delta h_{3}}{\Delta s_{3}}=\frac{P_{3} \Delta v_{3}}{R} \ldots \ldots \ldots 51$

6.18 Em vermelho tem-se a curva isobárica $s\left(h, P_{0}\right)$ e em azul a curva isobárica e isotérmica que caracteriza a região metaestável do fluido. . . . . . . . . 56

6.19 Em azul tem-se a temperatura de transição de fase, $T_{r e} \ldots \ldots \ldots \ldots \ldots \ldots \ldots$

6.20 Em azul tem-se o crle, e em verde crge.................... 58

6.21 À medida que a pressão aumenta, verifica-se a aproximação do binodais e a diminuição da concavidade da curva, como era esperado. . . . . . . . . . . 58

6.22 Fim da barreira em $T_{g s}=257.6 \mathrm{~K}$ para a pressão $P_{0}=70.11 \mathrm{kPa}$.

$6.23 \mathrm{O}$ cálculo de $n^{*}$ via função entropia $s\left(h, P_{0}\right)$ considerou $\phi=1$ e $C_{v}=3 R / 2$, na

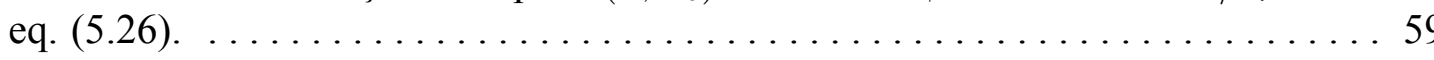

A.24 O loop de van der Waals no espinodal do líquido da EE-PR é mais profundo

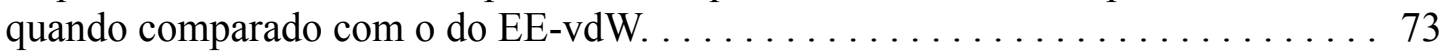

A.25 Curvas binodal da fase vapor da água desde o ponto triplo ao crítico. À direita tem-se a EE-vdW e à esquerda a EE-PR. . . . . . . . . . . . . . . . . . . . . 74

A.26 Curvas binodal da fase líquida da água desde o ponto triplo ao crítico. À direita

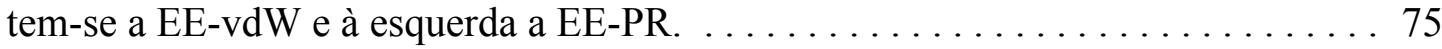

A.27 O desvio da EE-PR é significativo na fase líquida, enquanto que é muito grande o

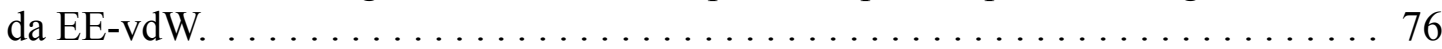

A.28 A falta de acurácia da EE-vdW mantém-se na região mestaestável. . . . . . . . . 76

B.29 Entropia do líquido e do gás ideais não traz o limite de estabilidade da matéria. . . . . 80

C.30 Representação vetorial dos três números iguais. $\ldots \ldots \ldots \ldots \ldots \ldots \ldots \ldots \ldots$

C.31 Superfície da eq. (C.20) tem a propriedade de ser mínima quando os três números

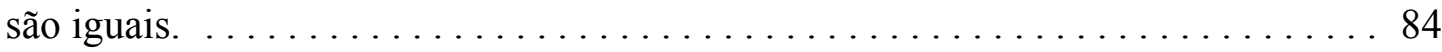

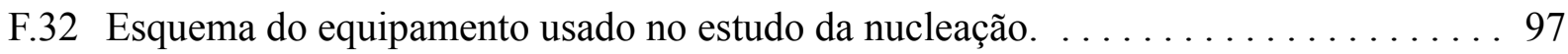

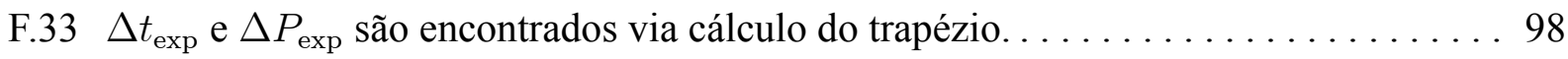


F.34 Os trabalhos de Strey et al. [33][36] mostram que o gás carregador não interfere na nucleação do vapor de água. . . . . . . . . . . . . . . . . . . . . . . . . 99

F.35 Independente do modelo clássico, $\partial \ln J / \partial \ln S_{s}=n^{*} \ldots \ldots \ldots \ldots \ldots \ldots$ 


\section{Lista de Tabelas}

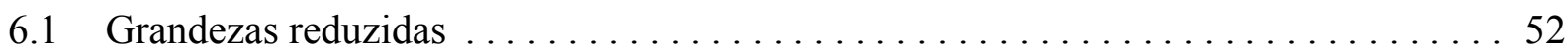

6.2 Pontos utilizados na construção dos sistemas $\Omega_{I}, \Omega_{I I}$, e $\Omega_{I I I} \ldots \ldots \ldots \ldots \ldots \ldots$

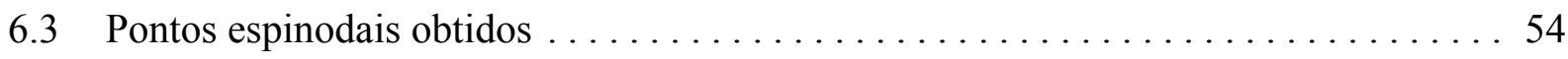

6.4 Pontos utilizados na construção dos sistemas $\ldots \ldots \ldots \ldots \ldots \ldots \ldots \ldots \ldots$

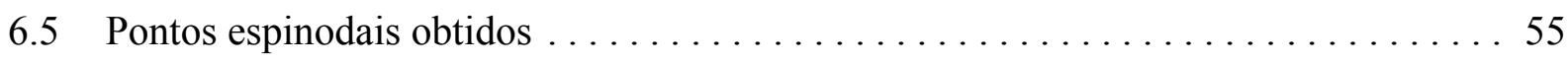

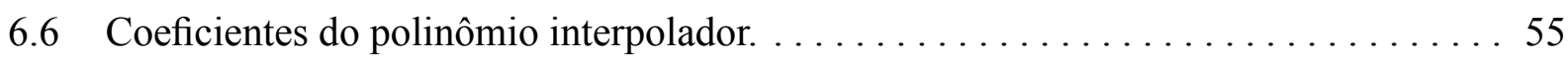

A.7 Constantes da equação de estados de van der Waals e de Peng-Robinson . . . . . . . 73 


\section{Simbologia}

A seguir é listado os principais símbolos usados ao longo do trabalho.

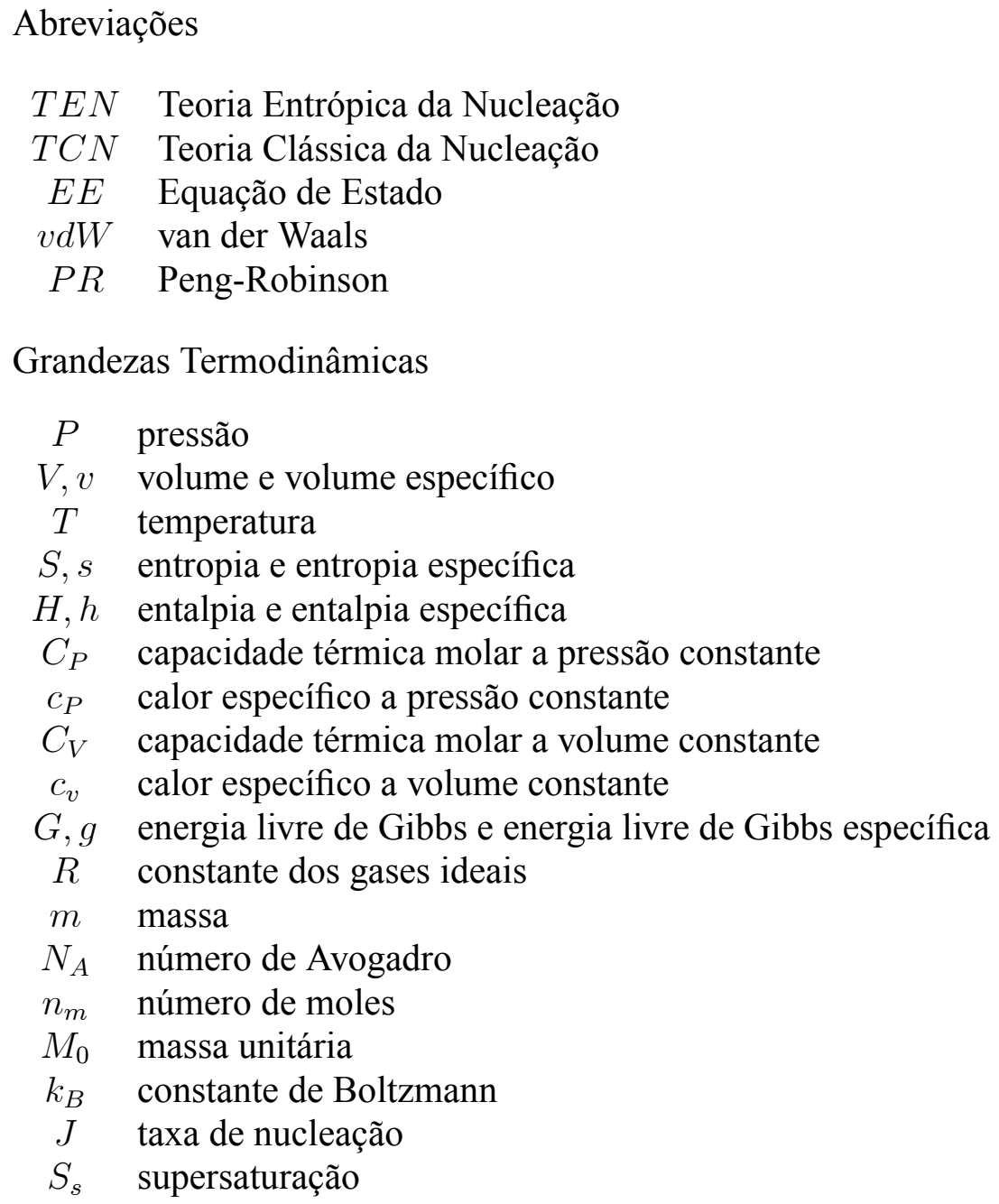

Outras definições

$n$ número de monômeros

$J$ taxa de nucleação

$\Gamma \quad$ ponto de um diagrama, $\Gamma(x, y, z)$

$\Omega$ sistema de equações

a coeficiente de superfície

$b$ coeficiente polinomial

c coeficiente polinomial reduzido

M matriz de coeficientes

N matriz de coeficientes pela regra de Cramer

b vetor coluna

y vetor solução

Subscritos 


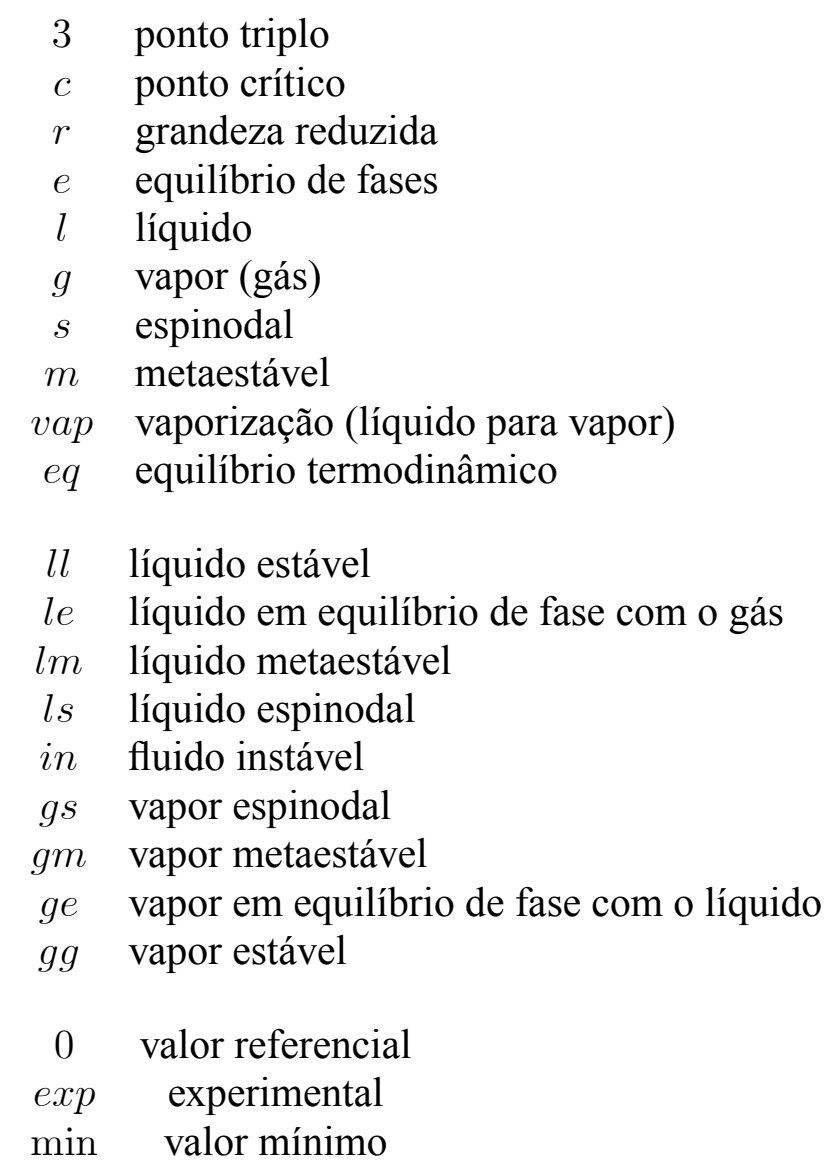

Sobrescritos

- processo reversível, gás ideal

* crítico

(I) Sistema de equações original

(II) Sistema de equações primeira derivada

(III) Sistema de equações segunda derivada

Operadores
$\Delta$ diferença finita
$d$ diferencial total
$\partial \quad$ diferencial parcial
$\int$ integral
$\sum$ somatório
$\prod$ produtório 


\section{Sumário}

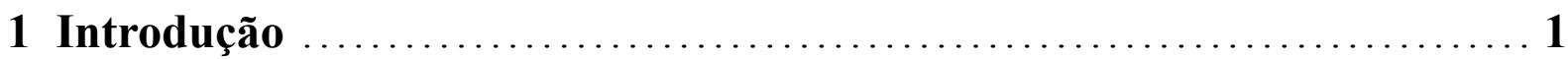

2 Considerações Teóricas ........................................ 4

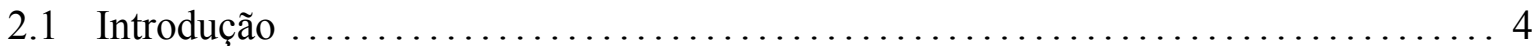

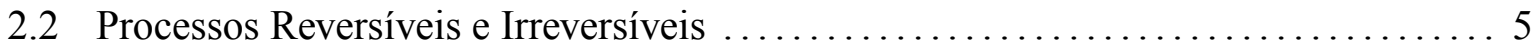

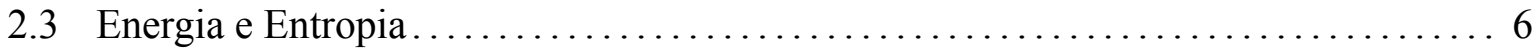

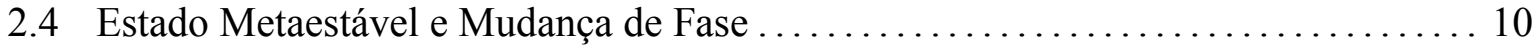

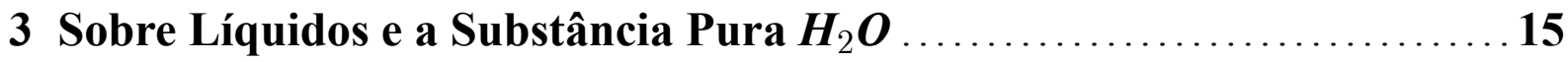

4 Sobre a Teoria Clássica da Nucleação ......................... 18

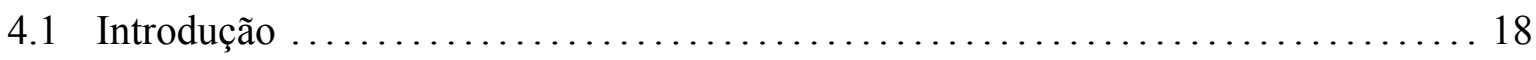

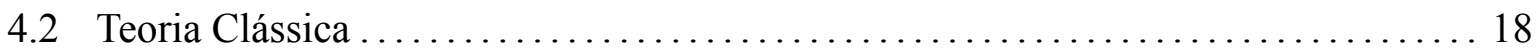

4.3 Deficiências na Teoria Clássica.................................... 20

4.4 Melhorias na TCN ................................................ 21

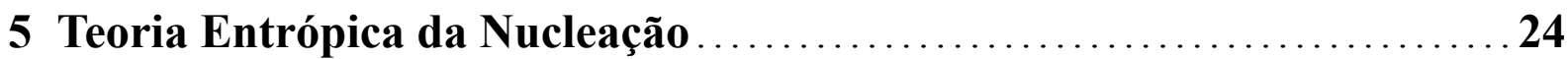

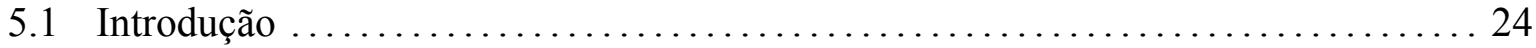

5.2 Fundamentação Teórica.......................................... 24

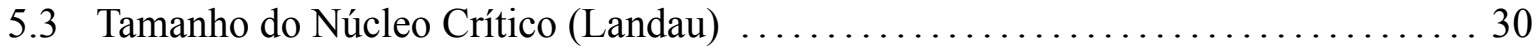

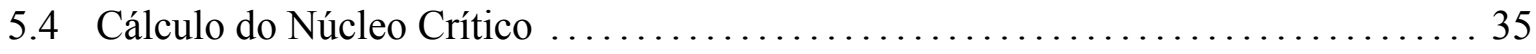

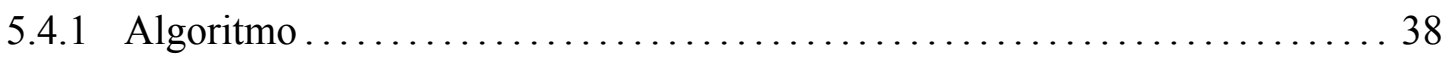

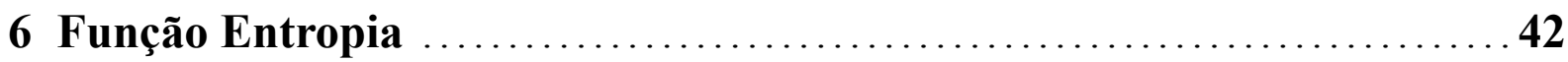

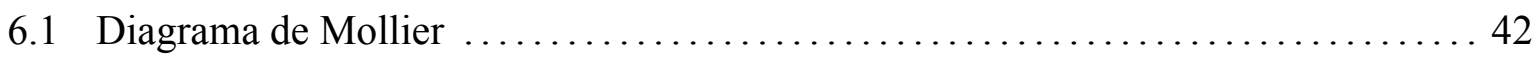

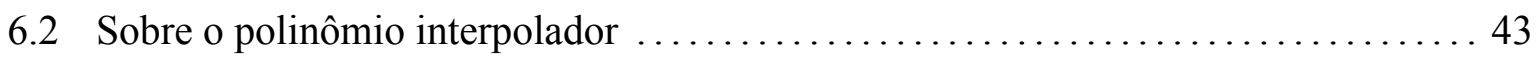




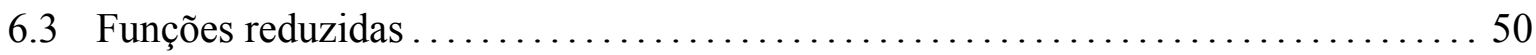

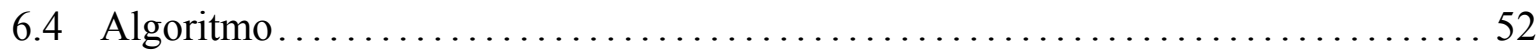

6.4.1 Sobre a obtenção dos dados experimentais ................... 52

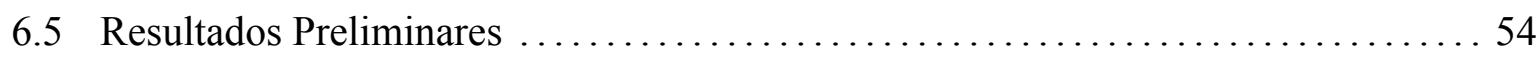

6.5 .1 Nucleação......................................... 57

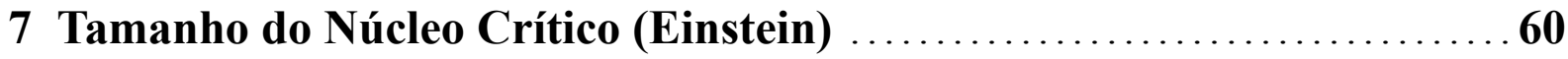

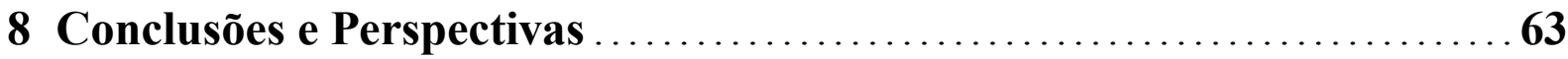

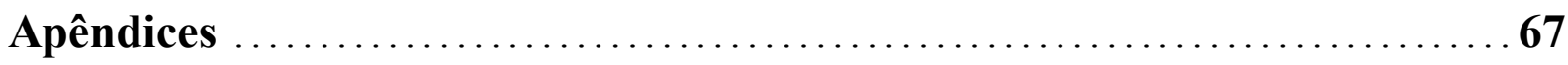

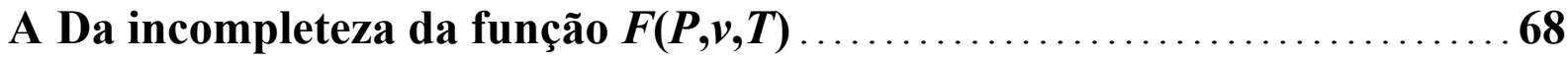

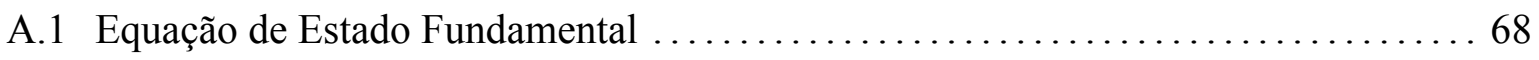

A.2 Das Equações de Estado de van der Waals e Peng-Robinson ................ 72

B Entropia de um Fluido Incompressível e de um Gás Perfeito .......... 77

C Problema dos três números iguais $\ldots \ldots \ldots \ldots \ldots \ldots \ldots \ldots \ldots \ldots \ldots \ldots \ldots 1$

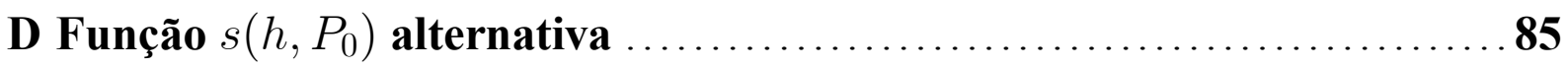

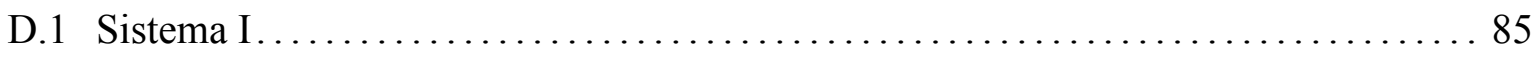

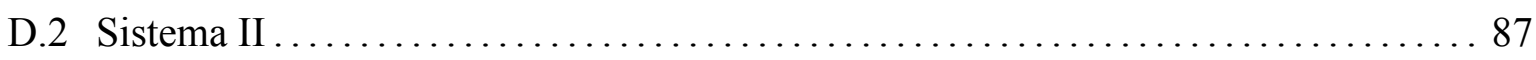

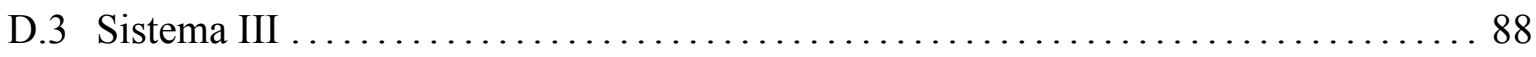

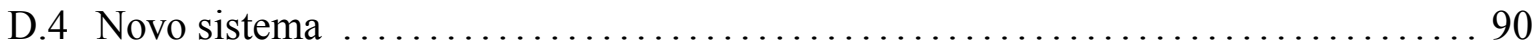

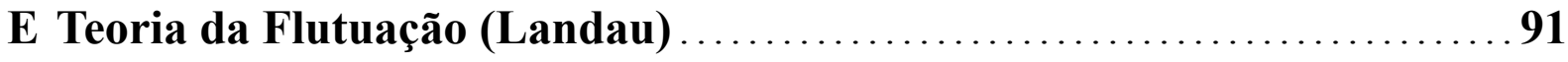

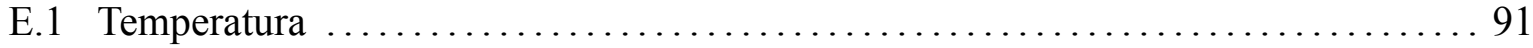

E.2 Entalpia.................................................. 94

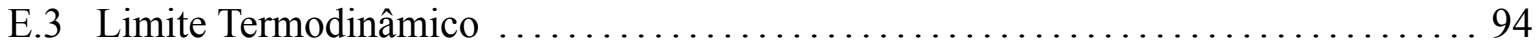

F Experimentos com a Nucleação da Água ..........................96 


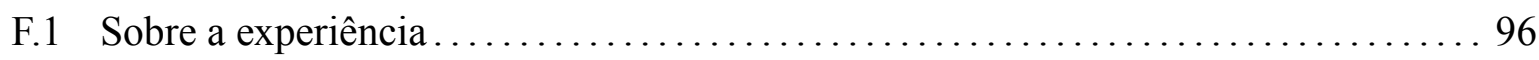

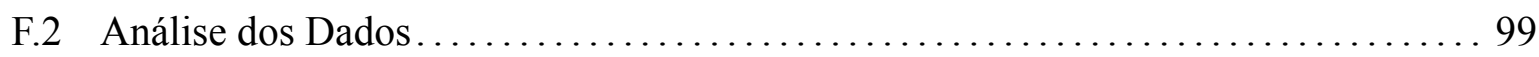

F.2.1 Resultados: Viisanen et al. ................................. 100

F.2.2 Resultados: Wölk-Strey .............................. 102

Referências................................................ 103 


\section{1 \\ Introdução}

Na natureza a matéria apresenta-se organizada em substâncias caracterizadas pela composição química e por um conjunto definido de propriedades físico-químicas. Uma substância é classificada como pura quando é composta de uma única composição química, não necessariamente de um único elemento químico. Substâncias compostas ou simplesmente misturas consistem em duas ou mais substâncias puras fisicamente misturadas.

Toda substância pode existir em três estados de agregação da matéria: sólido, líquido e gás. Josiah Willard Gibbs cunhou o termo fase para representar tais estados. Tem-se, portanto, as fases sólida, líquida e gasosa. Conforme Reiss [28], "uma fase é uma parte de um sistema, fisicamente distinta, macroscopicamente homogênea, de composição fixa ou variável. É mecanicamente separável do resto do sistema.”. Para produzir um sistema heterogêneo com quatro ou mais fases, é necessário que este consista de uma determinada mistura de substâncias puras.

Em um sistema consistindo de uma quantidade $m$ de massa de uma dada substância pura, a fase pode ser determinada a partir de seu estado termodinâmico. Verifica-se então que sob certas condições o sistema sofre mudança ou transição de fase de primeira ordem ${ }^{1}$ e em condições especiais duas ou mais fases podem coexistir, fala-se em equilibrio de fases. Experimentos mostram que uma substância pura pode exibir uma fase, uma mistura de duas fases e, no máx-

\footnotetext{
1 Na transição de fase de primeira ordem o surgimento da nova fase é caracterizada pela descontinuidade das variáveis extensivas como o volume específico. É observada em processos de mudança de estado de agregação da matéria como a fusão ou solidificação (sólido-líquido), vaporização ou condensação (líquido-gás), sublimação (sólido-gás). Existe a transição de fase de segunda ordem, caracterizada pela descontinuidade, p. ex., do calor específico à pressão constante ou, de maneira geral, na segunda derivada das grandezas termodinâmicas. É observada na reordenação dos átomos em redes cristalinas. 
imo, uma mistura de três fases ${ }^{2}$ [14]. Neste último caso, tem-se a coexistência das fases sólida, líquida e gasosa e diz-se que o sistema está no ponto triplo, $\Gamma_{3}$.

O fenômeno da transição de fase gás-líquido, conhecido como condensação (e seu oposto simétrico vaporização) tem como limite inferior o estado termodinâmico próximo ao ponto triplo. O limite superior é um estado próximo ao estado termodinâmico crítico, denominado ponto crítico, $\Gamma_{c}$; esse ponto é caracterizado pelas fases líquida e gasosa apresentarem o mesmo volume específico.

Em um processo de mudança de estado quase estático a transição de fase dá-se reversivelmente em proporção ao calor retirado (ou adicionado) através da linha de coexistência de fases ou de saturação. A completa transição de fase exige calor latente para sua complementação. O interesse desse trabalho diz respeito a um sistema constituído de uma substância pura colocado em um estado inicial metaestável da fase gasosa, devendo migrar para um estado final estável na fase líquida, liberando proporcionalmente a quantidade de calor necessária para a complementação do fenômeno. Porém, o vapor colocado nesse estado inicial metaestável não irá necessariamente condensar-se prontamente. A capacidade da fase inicial em permanecer um certo tempo em um estado metaestável é uma das mais notáveis características da transição de fase de primeira ordem, pois revela o fato deste estado estar separado do estado estável final por uma barreira de energia. Entende-se que o processo de transição de fase inicia-se com a formação de um aglomerado da nova fase ou núcleo, contendo $n$ monômeros ${ }^{3}$. Quando este núcleo contém uma quantidade mínima de monômeros, $n^{*}$, capaz de vencer a barreira energética, ele é denominado núcleo crítico. Uma vez iniciado o processo de nucleação, fala-se em taxa de nucleação, ou seja, o número de núcleos críticos formados por unidade de volume e de tempo.

\footnotetext{
2 A fase sólida de uma substância pura pode apresentar diferentes configurações alotrópicas, de modo que podem existir outros pontos triplos em que coexistam por exemplo duas fases sólidas e uma líquida, ou três fases sólidas.

3 Monômeros é o nome que designa elementos constitutivos da substância em estudo.
} 
Na Teoria Clássica da Nucleação (TCN) a taxa de nucleação homogênea, $J$, é dada por

$$
J=J_{0} \exp \left(-\frac{\Delta G^{*}}{k_{B} T}\right)
$$

onde $J_{0}$ é um pré-fator (relacionado a uma barreira cinética), $k_{B}$ é constante de Boltzmann, $T$ é a temperatura termodinâmica e $\Delta G^{*}$ é a barreira energética crítica, ou seja, o trabalho (mínimo) necessário para a formação de um núcleo crítico da nova fase (líquida) de tamanho $n^{*}$, quando exibe equilíbrio instável em relação à sua vizinhança (vapor) [13][33]. É natural portanto ter o trabalho crítico como proporcional ao núcleo crítico,

$$
\Delta G^{*} \propto n^{*}
$$

Conhecer a altura desta barreira de energia e/ou o tamanho do núcleo crítico é fundamental para a compreensão do processo de nucleação. É esperado que tal barreira energética diminua à medida que o estado metaestável aproxima-se do espinodal, quando então se anula, devido ao princípio de estabilidade termodinâmica - caracterizado por $\left(d^{2} s\right)_{e q}<0$, onde $s$ é a entropia e o subíndice $e q$ refere-se ao estado equilíbrio termodinâmico [10]. Paralelo a uma abordagem teórica que consiga estimar a barreira de energia crítica e/ou o núcleo crítico e, quiçá, a taxa de nucleação, é desejável uma equação de estado que forneça de forma realista o comportamento do fluido na região metaestável desde o binodal até o espinodal. 


\section{2 \\ Considerações Teóricas}

\subsection{Introdução}

A Termodinâmica é estruturada, essencialmente, sobre duas leis ou princípios fundamentais. A Primeira Lei da Termodinâmica é concebida postulando-se a existência de uma função de estado, a energia do sistema, E. Com a Segunda Lei da Termodinâmica postula-se a entropia, $S$, também como função de estado. A todo sistema físico pode-se associar uma energia $E$ e uma entropia $S$, o qual é definido em função de suas condições de contorno, que o diferencia da vizinhança ou ambiente (conforme ilustrado na Figura 2.1). A interação entre sistema e vizinhança, no que tange a troca de energia e/ou matéria, determina um conjunto de propriedades termodinâmicas que auxiliam na descrição do comportamento macroscópico daquele.

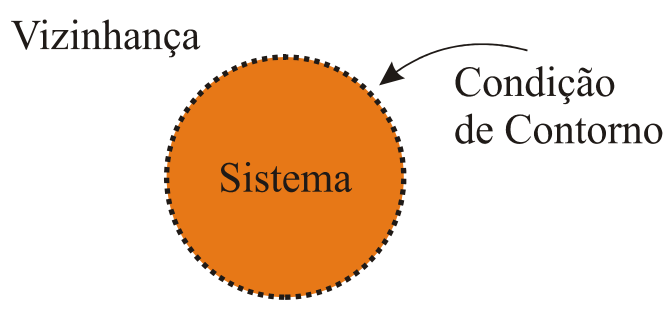

Fig. 2.1- O tipo de interação entre sistema e vizinhança é importante para a descrição do comportamento deste.

A experiência mostra que se um sistema físico está isolado, ou seja, não troca matéria e nem energia com a vizinhança, o seu estado evolui espontaneamente para um estado de equilíbrio devido a processos irreversíveis, que cessam no equilíbrio. Fala-se em Segunda Parte da Segunda Lei. Disto resulta que o sistema tornar-se-á invariante em relação ao tempo, ou seja, não se produzirá nenhuma mudança física ou química importante. Isto permite estabelecer a Lei 
Zero da Termodinâmica, a partir da qual considera-se a existência de uma temperatura termodinâmica (absoluta), T, como uma grandeza física; cujas medidas permitem estabelecer a noção de equilibrio térmico e de calor especifico. Neste sentido, postula-se energia e entropia como funções de estado de sistemas termodinâmicos em equilíbrio térmico, ou seja, que apresentam uma temperatura uniforme em toda sua extensão [27].

O problema básico em Termodinâmica de equilíbrio "consiste em determinar as características de estado de equilíbrio final quando o estado de equilíbrio inicial e a natureza dos vínculos são especificados" [14]. Porém o estado de equilíbrio inicial pode incluir estados de equilíbrio metaestável. Reiss [28] escreve que “a definição de equilíbrio termodinâmico não dá lugar para a distinção entre estado de equilíbrio metaestável e estável, visto que é exigido para tal que as propriedades macroscópicas do sistema não variem a uma taxa mensurável. Então flutuações locais afetam $P$ e $T$ (locais), mas não o equilíbrio termodinâmico. A única diferença entre o estado metaestável e o estado de equilíbrio estável está relacionada com o número de variáveis." Variáveis estas entendidas como vínculos internos do sistema. Entende-se então que no estado de equilíbrio metaestável o vínculo é uma barreira de energia, a qual é vencida devido a agitação mecânica e/ou devido a flutuações térmicas.

\subsection{Processos Reversíveis e Irreversíveis}

A mudança de estado de um sistema é realizada via processos reversíveis ou irreversíveis. O primeiro ocorre no limite da velocidade de transformação sendo nula (quasi-estático); pois a cada mudança de estado infinitesimal é necessário o restabelecimento do equilíbrio termodinâmico com as vizinhanças e no interior do próprio sistema. É, portanto uma idealização, que tem o papel de apresentar a temperatura definida a cada passo do processo (facilita o cálculo da entropia, mas não fornece a relação entre processos físicos e entropia produzida como a 
flecha do tempo). Já os processos irreversíveis são na verdade, a maioria de todos os processos termodinâmicos espontâneos que ocorrem na natureza.

A Termodinâmica de equilíbrio admite que toda transformação irreversível que se produz na natureza pode também ser obtida via um processo reversível. Ou seja, toda variação de entropia devido a uma transformação irreversível pode ser obtida por meio de um processo reversível, quasi-estático, implicando apenas uma troca de calor. Para um processo reversível, sistema e reservatório devem estar à mesma temperatura. No caso de um processo irreversível as temperaturas do sistema e do reservatório são diferentes.

Evidentemente que isto exclui processos que levam o sistema de um estado de equilíbrio estável a um estado de equilíbrio metaestável; esses devem ser suficientemente rápidos a fim criar vínculos internos no sistema, de modo que não basta que a troca de calor ocorra de forma quasi-estática. Entretanto, a termodinâmica de equilíbrio volta a ter validade depois de verificada a transição de fase.

\subsection{Energia e Entropia}

A variação de energia de um sistema, para qualquer processo adiabático, dada por

$$
\Delta E=-W_{a d}
$$

dependerá em geral de todas as suas propriedades independentes [14], em que $W_{a d}$ é o trabalho adiabático

$$
-W_{a d}=\Delta U+\Delta E_{k i n}+\Delta E_{p o t}+\Delta E_{e l}+\cdots
$$

onde $U$ é a energia interna, $E_{k i n}$ energia cinética, $E_{p o t}$ energia potencial gravitacional, $E_{e l}$ energia potencial elétrica entre outros. No caso de processos quasi-estáticos, sejam eles reversíveis ou irreversíveis, a continuidade dessas grandezas permite escrever

$$
d E=d U+d E_{k i n}+d E_{p o t}+d E_{e l}+\cdots
$$


Em particular, se o sistema permanece em repouso ou em movimento uniforme ao longo de uma linha eqüipotencial do campo gravitacional, e não há outros campos (como elétrico, magnético), então, $d E=d U$, ou seja, a variação da energia do sistema deve-se a processos internos que envolvem mudança de temperatura, de pressão e de volume, acompanhados ou não de mudança de fase e de reação química.

Se ao sistema é permitido interagir com a vizinhança através de paredes diabáticas, então, o calor trocado durante um processo reversível ou irreversível para ir do estado 1 para o 2 interfere na energia do sistema, de modo que isso é expresso como

$$
E_{2}-E_{1}=Q_{1,2}-W_{1,2}
$$

expressão esta que representa a Primeira Lei da Termodinâmica, onde $Q_{1,2}$ é o calor trocado. Naturalmente que no caso de paredes diabáticas $W_{1,2} \neq W_{a d}$. Se o processo é infinitesimal

$$
d E=d Q-d W
$$

Considerando agora que este sistema realiza um processo infinitesimal isobárico sem a presença de outros campos, a Primeira Lei é escrita como

$$
d U=d Q-P d V
$$

ou

$$
d Q=d(U+P V)
$$

pois a pressão é constante. Sendo a entalpia $H$ definida como

$$
H=U+P V
$$

pode-se escrever, assumindo que a pressão é hidrostática [28]

$$
d Q=d H
$$

Este é um resultado de interesse como será mostrado a seguir com a eq. (2.14). 
A variação da entropia de um sistema pode ser expressa de maneira geral como a soma de dois termos

$$
d S=d S_{e}+d S_{i}
$$

onde $d S_{e}$ representa a variação de entropia devido às trocas de calor com o exterior, e $d S_{i}$ é a variação da entropia devido a uma transformação não compensada produzida por processos irreversíveis internos do sistema; neste caso pode-se falar em produção de entropia devido a um trabalho dissipado. Está relacionada a gradientes de temperatura. Supondo um sistema isolado (em repouso e sem a influência de forças externas) composto de duas partes (Figura 2.2), cada qual a uma temperatura, $T_{0}$ e $T_{1}$ de modo que $T_{0}>T_{1}\left(=T_{0} / \alpha\right.$, em que $\left.\alpha>1\right)$, então a variação de energia (interna) de cada parte está ligada ao fluxo de calor devido ao gradiente de temperatura. Em acordo com a Primeira Lei, existe conservação de energia e por conseqüência de calor, $d Q=-d Q_{0}=d Q_{1}$. A produção de entropia do sistema pode ser escrita como a soma das entropias de cada parte,

$$
d S_{i}=\frac{d Q_{0}}{T_{0}}+\frac{d Q_{1}}{T_{1}}=\left(-\frac{1}{T_{0}}+\frac{1}{T_{0} / \alpha}\right) d Q
$$

$\mathrm{ou}$

$$
d S_{i}=\frac{1}{T_{0}}(\alpha-1) d Q
$$

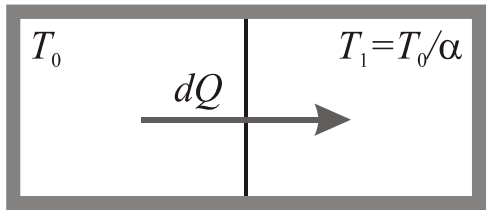

Fig. 2.2- A produção de entropia é observada quando há gradiente de temperatura $T_{0}>T_{1}\left(=T_{0} / \alpha\right.$, onde $\alpha>1$ ).

Dessa forma, o chamado princípio do aumento da entropia é expresso como

$$
T_{0} d S_{i}=(\alpha-1) d Q>0
$$


para $\alpha=1$ leva a $d S_{i}=0$, de modo que não há produção de entropia; no caso de um sistema que troca calor com a vizinhança, esse é o caso limite de um processo reversível. Para $\alpha>1$ tem-se $d_{i} S>0$ implicando em processo irreversível. Nota-se que $\alpha=1$ é o limite.

Um sistema constituído de paredes diabáticas em contato com um banho térmico à temperatura $T_{0}$, em que um processo termodinâmico ocorre isobaricamente, a variação de entropia com ajuda da eq. (2.9) é dada por

$$
d S_{e}=\frac{d Q}{T_{0}}=\frac{d H}{T_{0}}
$$

onde $H$ é a entalpia.

Dessa forma, a Primeira Parte da Segunda Lei da Termodinâmica é escrita como

$$
d S=\frac{d H}{T_{0}}+\frac{(\alpha-1) d Q}{T_{0}}
$$

ou

$$
T_{0} d S=d H+(\alpha-1) d Q
$$

A troca de calor com o reservatório térmico é fundamental para o fluido mudar da fase gasosa para a fase líquida. A transição de fase a partir de um estado metaestável é um processo irreversível e, no caso da condensação do fluido, é esperado uma produção de entropia no reservatório de temperatura.

A equação (2.16) pode ser reorganizada como

$$
-(\alpha-1) d Q=d H-T_{0} d S
$$

Se a temperatura for constante pode-se identificar

$$
-(\alpha-1) d Q=d G
$$

onde $G$ é a energia livre de Gibbs, visto que por definição, $G=H-T S$. Um problema de interesse é determinar o trabalho para levar o sistema do estado 1 com $T_{1}=T_{0}$ para o estado 2 com $T_{2}=T_{0}$, sendo permitido assumir qualquer temperatura durante o processo [14]. Diante do exposto, parece natural estudar esse trabalho a partir das variáveis entalpia e entropia em 
um sistema mantido a pressão constante. Além disso, processos isobáricos são de interesse experimental.

\subsection{Estado Metaestável e Mudança de Fase}

A natureza física da transição de fase gás-líquido é suficientemente bem ilustrada com a análise do comportamento de fluidos que obedecem à equação de estado de van der Waals (vdW) [14][13]. Sua forma reduzida

$$
P_{r}=\frac{8 T_{r}}{3 v_{r}-1}-\frac{3}{v_{r}^{2}}
$$

é denominada equação reduzida ${ }^{4}$ de vdW. É uma equação universal no sentido de que não depende explicitamente das características do fluido em estudo e também não depende explicitamente do número de partículas contidas no sistema. Sua forma, porém, exibe a continuidade de estados gasoso e líquido, como mostrado a seguir 5 .

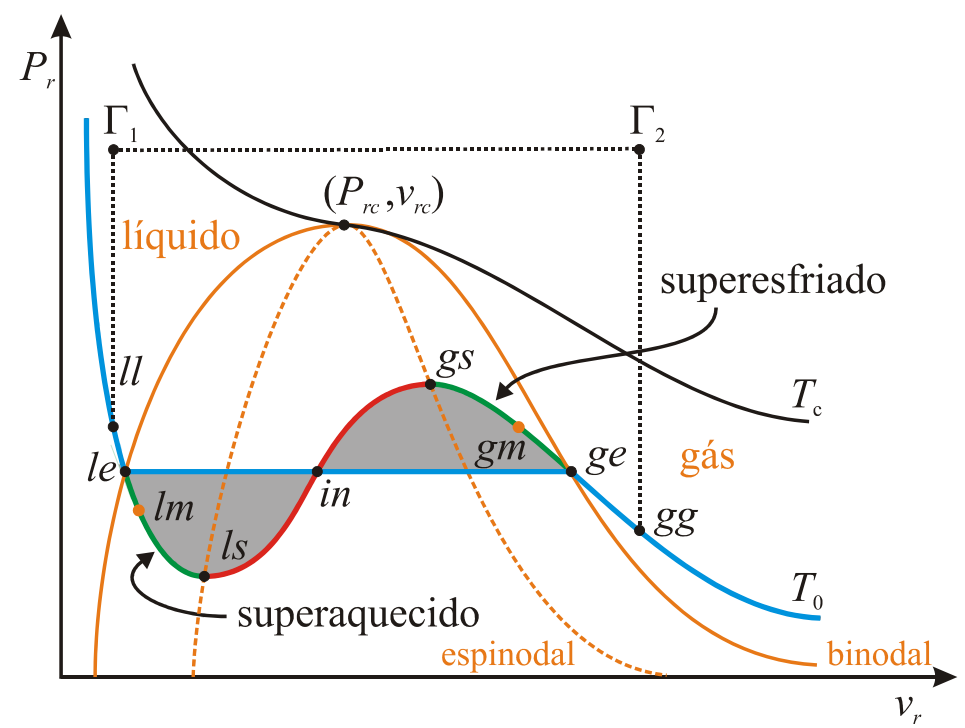

Fig. 2.3- A linha laranja contínua representa o líquido e o vapor saturado (binodal); a pontilhada define o limite de estabilidade para fase líquida e gasosa (espinodal).

4 Aqui, as grandezas reduzidas são definidas como $P_{r}=P / P_{c}, v_{r}=v / v_{c}, T_{r}=T / T_{c}$, onde $P_{c}, v_{c}$ e $T_{c}$ são respectivamente a pressão, o volume e temperatura no ponto crítico.

5 GUGGEnHEIM, E. A. Thermodynamics. $6^{\text {th }}$ ed. Oxford: North-Holland, 1977. (§ 3.45). 
O diagrama $P, v$ mostrado na Figura 2.3 (em coordenadas reduzidas) exibe duas isotermas típicas: uma crítica $\left(T_{c}\right)$ e uma subcrítica $\left(T_{0}\right)$. Como exemplo seja um sistema com uma substância pura como a água, em que a temperatura é fixada em $T_{0}=373 \mathrm{~K}$ no estado estável $l l$ cuja pressão $P_{l l}>101.325 \mathrm{kPa}\left[=P_{e}=P\left(T_{0}\right)\right]$. Diminuindo a pressão até $P_{e}=101.325 \mathrm{kPa}$, ao longo do segmento de curva $\overline{l l} l e$, surge a primeira bolha de vapor em le. A partir deste ponto, mantendo $T_{0}$ e $P_{e}$ constantes, a adição de calor simplesmente produz quantidade adicional de vapor; tal calor é usado na mudança estrutural (quebra de ligações moleculares), não afetando sua temperatura. Com a quantidade de calor adicionada igual ao calor latente específico de vaporização, $L_{v a p}(=2242.11 \mathrm{~kJ} / \mathrm{kg})$, o sistema é conduzido ao longo do segmento de reta $\overline{l e ~ g e}$ (linha de saturação) até o ponto ge, quando a última gota do líquido é vaporizada. Com o fluido agora na fase gasosa, a diminuição da pressão até o ponto $g g$, ao longo do segmento de curva $\overline{g e g g}$ implicará na expansão do mesmo.

O líquido no estado antes de iniciar a vaporização é chamado saturado (le). Quando a última gota é vaporizada, o estado é de vapor saturado (seco) ( ge). O trabalho de expansão do fluido durante a coexistência de fases (de le a $g e$ ) a $T_{0}, P_{e}=P\left(T_{0}\right)$ contantes é uma pequena fração de $L_{v a p}$. O sistema com as fases líquida e gasosa coexistindo em equilíbrio é chamado saturado [14].

Aumentando-se a temperatura com a qual a substância pura é vaporizada, observa-se que a diferença entre os volumes específicos das fases líquida e gasosa $\left(v_{g e}-v_{l e}\right)$ diminui até alcançar o valor zero em uma determinada pressão denominada crítica $P_{c}$. O ponto em que se observa esse estado é denominado ponto crítico, $\Gamma_{c}=\left(P_{c}, T_{c}, v_{c}\right)$, quando verifica-se $v_{c}=v_{g c}=v_{l c}$.

A transição de fase, como descrita acima, que ocorre sobre a linha de equilíbrio de fases, é reversível e é simétrica no calor latente específico $\left(L_{v a p}\right)$. Todas as transições de fase têm em comum o fato de terem um grau de liberdade ( $P$ ou $T$, parâmetros intensivos) e exigirem calor latente para sua complementação. 
O vapor aquecido acima de sua temperatura de saturação a uma dada pressão é chamado superaquecido; e o líquido esfriado abaixo de sua temperatura de saturação a uma dada pressão é chamado superesfriado. Uma substância na fase vapor que se encontra em um estado metaestável (em relação à fase líquida) é conhecida como supersaturada ou superesfriada. E a fase líquida no estado metaestável (em relação à fase gasosa) é tida como supersaturada ou superaquecida.

$\mathrm{Na}$ isoterma $T_{0}$ da Figura 2.3, o segmento de curva anterior ao ponto le, representa o líquido estável, $\overline{l e g e}$ é isobárico e isotérmico, o qual indica a transição de fase por separação de fases (processo quase-estático); os segmentos de curva $\overline{l e l s}$ e $\overline{g s g e}$, indicam regiões de metaestabilidade, ou seja, líquido e vapor supersaturados, respectivamente; e em $\overline{l s g s}$, temse a região de instabilidade. Os pontos le e ge são conhecidos como binodal e os pontos ls e gs como espinodal. As curvas binodal (curva laranja inteira) e espinodal (curva laranja pontilhada) delimitam as regiões de estabilidade, de metaestabilidade e de instabilidade. $\mathrm{Na}$ isoterma crítica os pontos binodal e espinodal equivalem-se, ou seja, as fases líquida e gasosa são indistinguíveis.

Cabe notar que o diagrama $P, v$ também exibe a artificialidade da distinção entre as fases líquida e gasosa. É possível, manipulando $P$ e $v$, levar o sistema do estado $l l$ para o estado $g g$ sem que haja transição de fase [11]. Comprimindo o fluido aproximadamente a volume constante desde o estado $l l$ até o ponto $\Gamma_{1}$ (vide Figura 2.3) em seguida expandi-lo isobaricamente até $\Gamma_{2}$ (adicionando-lhe calor) e, por fim, diminuindo a pressão isocoricamente até o estado $g g$. Tem-se que o sistema mudou de fase sem apresentar o fenômeno da transição de fase.

O processo de evaporação ou, o reverso, de condensação nem sempre ocorre sobre a linha de coexistência de fases (segmento de reta $\overline{l e g e}$, na Figura 2.3). Quando um vapor é esfriado com alguma agitação e/ou quando corpos estranhos, como partículas de poeira, partículas ionizadas, ou pequenas gotas do líquido estão presentes nele, a condensação inicia-se quando 
a pressão $P$ e a temperatura $T$ simultaneamente alcançarem valores que encontram-se sobre a curva pressão-vapor (vide Figura 3.4). Quando o processo é examinado em maior detalhe, verifica-se que as partículas estranhas serviram de núcleo de condensação para iniciar o processo. Nesta situação é irrelevante se a curva pressão-vapor é alcançada à pressão constante, à temperatura constante, ou via alguma outra forma, por exemplo, por expansão adiabática, pois o único critério é a combinação de temperatura e pressão [14].

Quando os núcleos de condensação são cuidadosamente removidos e quando o processo ocorre rapidamente, mas sem demasiada agitação (mínimo possível), o sistema parece desenvolver uma certa resistência interna à condensação, e permanece no estado gasoso quando deveria separar-se nas fases gasosa e líquida. Pode ficar neste estado por um determinado intervalo de tempo, que pode ser suficientemente longo. Porém, agitação vigorosa (ou a introdução de núcleo de condensação) provoca a espontânea condensação de parte do vapor e o sistema assume um estado de equilíbrio, ou seja, estado de coexistência de fases [14]. Processos termodinâmicos como estes dão realidade física aos segmentos de curva $\overline{l e l s}$ e $\overline{g s g e}$ na Figura 3.4, previstos pela eq. (2.19), daí a continuidade de estados gasoso e líquido. Tal segmento de curva também é conhecido como loop de van der Waals.

Embora o estado do qual evolve as duas fases de um sistema é coloquialmente referido como estado estável, um cilindro contendo semelhante sistema de duas fases não estará necessariamente em um estado estável. Suponha que tal sistema esteja contido em um cilindro com pistão, de modo a assegurar constante a pressão, e mantém-se em contato térmico com um reservatório de calor. Se o pistão é deslocado para cima ou para baixo, não retornará à sua posição original, mas permanecerá na posição deslocada. Durante o processo, algum calor é trocado e algum vapor irá condensar-se ou algum líquido irá evaporar-se. Então, o sistema estará em um equilíbrio neutro com relação a tal distúrbio ( $\Delta h$ devido a $\Delta v)$. Contrariamente, a adição ou extração de uma pequena quantidade de calor causará o deslocamento do pistão, e o sistema 
continuará a estar em um equilíbrio neutro ( $\Delta v$ devido a $\Delta h)$. Em contraste, se a atual temperatura de saturação é alterada por um pequeno aumento, $T_{e} \longrightarrow T_{e}+\Delta T$, então o equilíbrio será perturbado. As duas fases não podem coexistir em tais condições e, no caso, o líquido vaporizar-se-á completamente, ou seja, não apresentará separação de fases. Similarmente, se $T_{e} \longrightarrow T_{e}-\Delta T$, então todo vapor condensar-se-á. Conclui-se que o sistema encontra-se em equilíbrio instável com relação a este tipo de perturbação: flutuações de energia. O mesmo vale para alterações na pressão. Contudo, o estado de coexistência de duas fases constitui o estado estável com respeito a um estado metaestável que foi perturbado por agitação mecânica [14].

Estados metaestáveis possuem vínculos internos que são removidos pela agitação, pela introdução de núcleos de condensação, ou por flutuações da energia, caso de interesse deste trabalho. A transição de um estado metaestável para um estado estável é análogo a um processo irreversível que ocorre devido à remoção de um vínculo interno do sistema. No presente caso, o vínculo interno é de natureza sutil: barreira de energia $\left(\Delta G^{*}\right)$. A barreira é vencida com a formação de um núcleo estável da nova fase, cujo tamanho está relacionado com o estado metaestável do sistema.

O processo de nucleção é irreversível, onde a entropia é uma assinatura natural, e é necessário troca de calor latente específico com a vizinhança para completar a transição. E se o processo é isobárico, é natural que as grandezas entropia, entalpia e pressão sejam consideradas na sua descrição. 


\section{3 \\ Sobre Líquidos e a Substância Pura $\mathrm{H}_{2} \mathrm{O}$}

Em termos de organização molecular das três fases da matéria, de um lado tem-se o sólido que é caracterizado por ser denso e cristalino. Seus átomos ou moléculas constituintes são associados a uma dada posição no espaço, onde o único movimento possível é de natureza oscilatória, centrada em torno da posição de equilíbrio. No outro lado, um gás ideal apresenta suas moléculas livres para moverem-se randomicamente. Entre esses extremos existem os líquidos. Pode-se descrevê-los aproximadamente a partir de um sólido perturbado no qual o grau de ordem foi reduzido ao ponto de liquefação, de modo que as moléculas ocupam as mesmas posições em determinado tempo médio (técnicas de raio-X e difração de nêutrons sobre os líquidos têm evidenciado características estruturais de sólidos) [9]; ou pode-se descrevê-los a partir de um gás denso no qual o movimento molecular é limitado pela proximidade de outras moléculas e sujeito a considerável grau de interação molecular. Contudo, uma definição para líquido é tê-lo como um fluido autocondensado que não suporta ser expandido indefinidamente [11].

Uma substância pura de particular interesse é a água, único líquido inorgânico que ocorre naturalmente na Terra. O grande intervalo de temperatura da fase líquida (de $T_{3}$ a $T_{c}$ ) é uma indicação da presença de forças de longo alcance na água. Isto é confirmado pelo pequeno calor latente de fusão (em torno de $15 \%$ do calor latente de vaporização) e sugere que o líquido mantém muito da ordem do estado sólido e que esta ordem é destruída somente no ponto de ebulição [9]. Uma notável característica e, provavelmente a maior propriedade anômala da água líquida, é sua grande capacidade térmica ser reduzida à metade na solidificação ou na ebulição.

O principal interesse deste trabalho encontra-se na região da chamada curva pressão-vapor ou de saturação (vide Figura 3.4) em que se verifica a coexistência das fases líquida e gasosa. 
A equação característica desta curva é a equação de Clausius-Clapeyron, dada por

$$
\left(\frac{d P}{d T}\right)_{e}=\frac{L_{v a p}}{T_{e} \Delta v}
$$

onde $L_{v a p} / T_{e}=s_{g e}-s_{l e}$, em que $L_{v a p}=h_{g e}-h_{l e}$ é o calor latente específico de vaporização que depende da pressão a que o sistema está submetido e $\Delta v=v_{g e}-v_{l e}$. Na Figura 3.4 tem-se parte do diagrama de fases experimental da água pura, no qual é destacado a curva pressão-vapor em que ocorre a coexistência líquido-vapor; tem como limite os pontos triplo, $\Gamma_{3}=\left(P_{3}, v_{3}, T_{3}\right)$, e crítico, $\Gamma_{c}=\left(P_{c}, v_{c}, T_{c}\right)$.

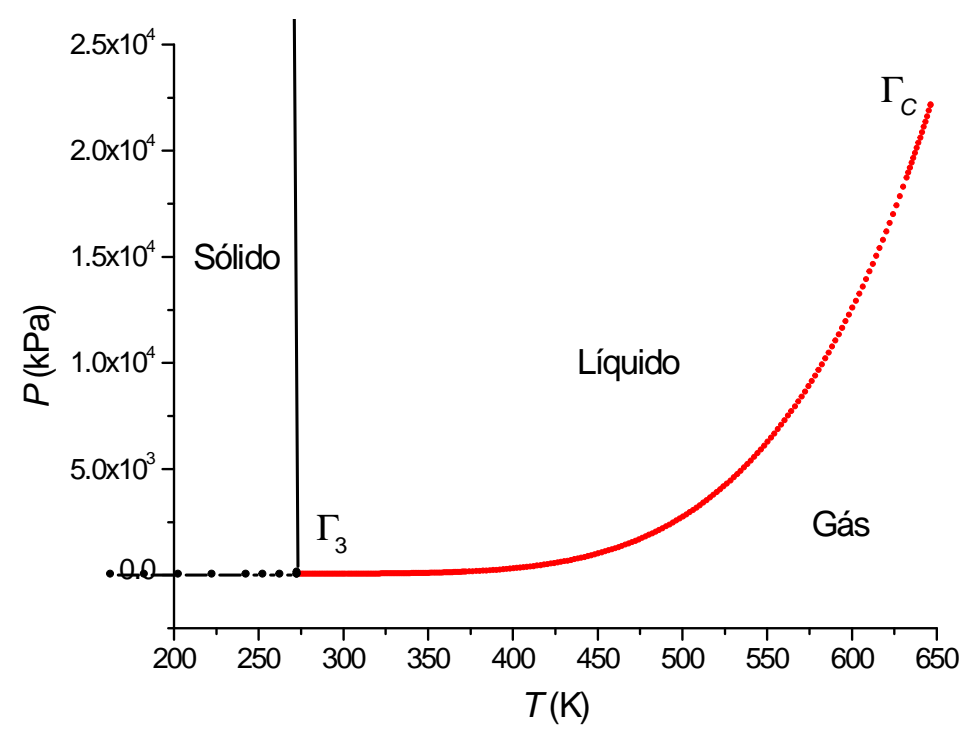

Fig. 3.4- Diagrama de fases experimental da água pura, no qual se encontra evidenciada a curva pressão-vapor: coexistência das fases líquida e vapor.

No ponto triplo verifica-se a coexistência de três fases, sólida, líquida e gasosa. É observada quando o sistema encontra-se no estado em que $T_{3}=273.16 \mathrm{~K}, P_{3}=6.107 \times 10^{2}$ $\mathrm{Pa}$ - e os volumes específicos do líquido e do gás são, respectivamente, $v_{l 3}=1.0002 \times 10^{-3}$ $\mathrm{m}^{3} / \mathrm{kg}$ e $v_{g 3}=206.3 \mathrm{~m}^{3} / \mathrm{kg}$. O ponto crítico é caracterizado por um estado em que temperatura e pressão são tais que o volume específico do líquido é igual ao do vapor, $v_{l c}=v_{g c}$, de modo que não se pode distinguir uma fase da outra . Para a água, a temperatura crítica é $T_{c}=647.31$ 
$\mathrm{K}$, a pressão crítica é $P_{c}=2.21 \times 10^{7} \mathrm{~Pa}$ - e o volume específico crítico para ambas as fases é $v_{l c}=v_{g c}=3.18 \times 10^{-3} \mathrm{~m}^{3} / \mathrm{kg}$. 


\section{4 \\ Sobre a Teoria Clássica da Nucleação}

\subsection{Introdução}

A teoria para descrever o fenômeno da transição de fase de primeira ordem, conhecida como Teoria Clássica da Nucleação (TCN), teve origem nos estudos da capilaridade. Na primeira metade do século XX, R. Becker e W. Döring, aperfeiçoaram o modelo para predições quantitativas do fenômeno da nucleação. Ainda que de acurácia questionável, lançou luz sobre um fenômeno físico de importância tecnológica. Com o avanço da tecnologia, medidas mais acuradas mostraram inconsistências na TCN. Esta apresenta estranha dependência com a temperatura em que consegue representar o fenômeno em estreita faixa de temperatura. Várias correções têm sido propostas. Dentre as mais recentes destacam-se a teoria semifenomenológica de A. Dillman e G. E. A. Meier [4][5] de acurácia melhor que a clássica, em que foram usadas técnicas empíricas e numéricas; e a teoria auto-consistente proposta por H. Reiss, W. K. Kegel e J. L. Katz [29].

\subsection{Teoria Clássica}

O sistema em que acontece a nucleação consiste em uma mistura de duas partes homogêneas (fase inicial vapor e fase final líquida) separadas por uma superfície matemática bem definida. Fala-se em tensão superficial do núcleo (interface). O trabalho para a formação de um aglomerado (suposto esférico) em função do raio

$$
\Delta G(r)=-\frac{4 \pi}{3 z_{18}} r^{3} \Delta \mu+4 \pi \sigma r^{2}
$$


onde $v_{0}$ é o volume de um monômero, $\mu$ é o potencial químico e $\sigma$ representa a energia de superfície, independente do número de monômeros no aglomerado. O emprego de $\sigma$ é herança dos estudos da capilaridade, razão pela qual a TCN é também chamada de Teoria Capilar da Nucleação. O primeiro termo do lado direito na eq. (4.1) é interpretado como energia volumétrica e o segundo como energia superficial do aglomerado.

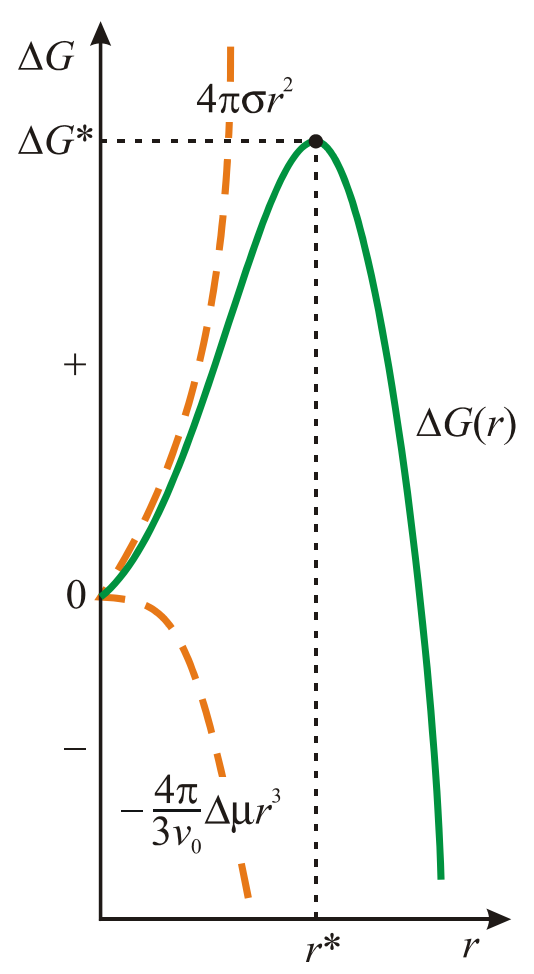

Fig. 4.5- O pico é caracterizado pelo equilíbrio entre as energia de volume e de superfície: núcleo crítico.

A Figura 4.5 mostra o comportamento da eq. (4.1) em função do raio do aglomerado. O ponto de máximo representa o trabalho crítico determinado pelo raio crítico. Aglomerados menores que o raio crítico, $r<r^{*}$ decaem (domínio da energia de superfície), minimizando a energia livre de Gibbs; se $r \geq r^{*}$ crescem (domínio da energia de volume), também minimizando a energia livre.

Assumida que a formação de aglomerados se dá num regime estacionário (modelo Szilard) [13][23], a distribuição de equilíbrio do tipo Boltzmann do número de aglomerados de 
tamanho $n$ por unidade de volume,

$$
N^{(e)}(n)=N^{(e)} \exp \left(-\frac{\Delta G^{*}}{k_{B} T}\right)
$$

correspondente a um mínimo de energia livre de Gibbs, onde $N^{(e)}=N^{(e)}(1)$ refere-se é a densidade de monômeros $(n=1)$ na fase inicial, portanto vapor. Em relação ao tempo, essa densidade é escrita como taxa de nucleação dada por

$$
J=J_{0} \exp \left(-\frac{\Delta G^{*}}{k_{B} T}\right)
$$

onde $J_{0}$ é um pré-fator assumido constante. Na abordagem de Becker-Döring a eq. (4.3), $J=J_{B D}$, é introduzida como teorema [13] onde o pré-fator é dado por

$$
J_{0}=v_{0}\left(\frac{P}{k_{B} T}\right)^{2}\left(\frac{8 \sigma}{\pi m_{0}}\right)^{1 / 2}
$$

onde $P$ é a pressão e $m_{0}$ é a massa de um monômero.

\subsection{Deficiências na Teoria Clássica}

Apesar de já se ter compreendido muito da cinética da transição de fase de primeira ordem, diversos problemas na formulação da teoria, bem como na aplicação da teoria em casos particulares, permanecem em aberto. Citando alguns:

- É esperado que para $n=1, N^{(e)}(1)=N^{(e)}$, ou seja, não há aglomerados no sistema, resultado este não obtido com Becker-Döring.

- É predito a dependência da taxa de nucleação $J_{B D}$ com a supersaturação $S_{s}$ e com a temperatura $T$, ou seja, $J=J\left(S_{s}, T\right)$. Em torno de $T=240 \mathrm{~K}$ e de $P=400 \mathrm{kPa}$ as predições quantitativas concordam com a experiência. No entanto, a TCN mostra estranha dependência com a temperatura, isto é, as taxas de nucleação encontradas em $J_{B D}$ comparadas com a experiência são altas a temperaturas mais elevadas e baixas a temperaturas menores [33] [36]; 
- A TCN contém um erro fundamental em que prediz uma barreira finita de energia para que haja nucleação mesmo no espinodal, sendo que a propriedade central neste estado é o desaparecimento desta barreira com o fluido passando de um estado termodinamicamente metaestável para um estado instável [20].

- Nucleação e crescimento procedem sem decréscimo da força motriz em violação ao princípio de Châtellier-Braun ${ }^{6}$ [20]. Com este princípio, além de afetar o calor latente do sistema, tem-se o indicativo de que a temperatura não deve ser constante durante a nucleação;

- Simulações computacionais e experimentos sofisticados indicam que a interface não é bem definida e sim difusa ou podendo apresentar estrutura fractal (principalmente para pequenos embriões e núcleos) [20];

- Deve ser garantido que a quantidade condensada de substância seja imediatamente substituída por processo de difusão (modelo de Szilard para nucleação homogênea) [12] [20][13]. Evidentemente que num caso real há depleção do material, pelo menos localmente.

\subsection{Melhorias na TCN}

A teoria de A. Dillmann e G. E. A. Meier (DM) [4][5] combina a taxa de nucleação na forma generalizada proposta por McDonald ${ }^{7}$ com o modelo semifenomenológico da gota proposto por Fisher $^{8}$ para a energia livre de Gibbs na formação de um aglomerado. O modelo da gota permite

6 Este princípio diz que a ação exterior que tira o corpo do estado de equilíbrio térmico, estimula processos neste que tendem a contrapor esta ação debilitando o resultado da mesma. Assim, por exemplo, o aquecimento do corpo estimula processos relacionados com a absorção de calor e, vice-versa, o esfriamento contribui para o surgimento processos em que se desprende calor [16].

7 McDOnAld, J. E. Am. J. Phsys, 30, 870, 1962; 31, 31, 1963; apud Dilmman-Meier [4][5].

8 FISHER, M. E. Physics, 3, 255, 1967; apud Dilmman-Meier [4][5]. 
graus de liberdade translacional, rotacional, vibracional e configuracional, bem como a variação da tensão de superfície com o tamanho do aglomerado. Essa teoria contém a TCN como caso especial. Todos os parâmetros livres do modelo podem ser determinados a partir de quantidades macroscópicas bem conhecidas. Essa teoria fornece predição da taxa de nucleção muito melhor que a TCN (vide Figura 4.6).
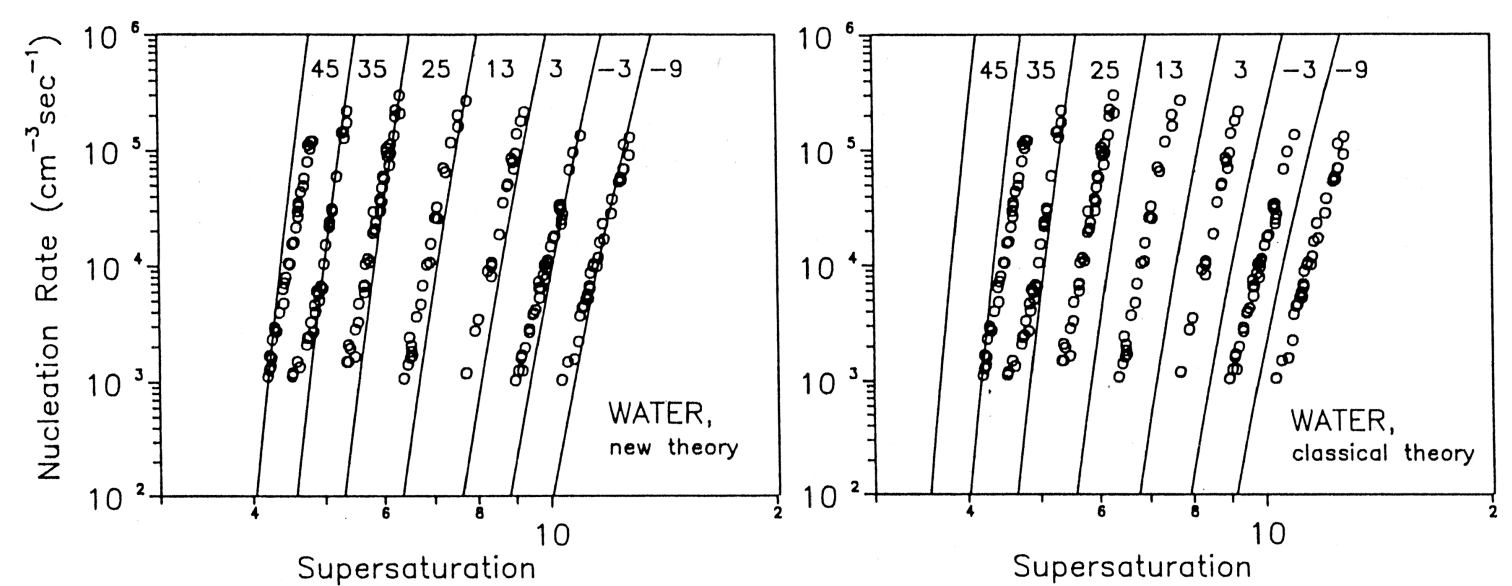

Fig. 4.6- À esquerda a teoria de Dillmann-Meier encontra-se em melhor concordância com a experiência; à direita a estranha dependência da TCN com a temperatura. A temperatura é dada em graus Celsius [4].

No trabalho de H. Reiss, W. K. Kegel e J. L. Katz [29] a energia livre de Gibbs é reescrita acrescentando um termo referente à entropia de mistura de fases, em que consideram o sistema com densidade homogênea de aglomerados. Escrevem a taxa de nucleação como

$$
J_{R K K}=\frac{R}{S_{s}} J_{B D}
$$

onde $J_{B D}$ é a taxa de nucleação dada por Becker-Döring, $R$ é um fator obtido com o reposicionamento da energia livre e $1 / S_{s}$, o inverso da supersaturação destina-se a satisfazer a lei de ação das massas com respeito à concentração de monômeros não-aglomerados. Com isso, os autores conseguiram corrigir uma "inconsistência interna" na TCN ou seja, para $n=1$, $N^{(e)}(1)=N^{(e)}$. Com essa abordagem a predição da taxa de nucleação, corrigida por um fa- 
tor 6000 ( $a d$ hoc), melhorou consideravelmente como mostra a Figura 4.7. Porém, persiste a estranha dependência com a temperatura.

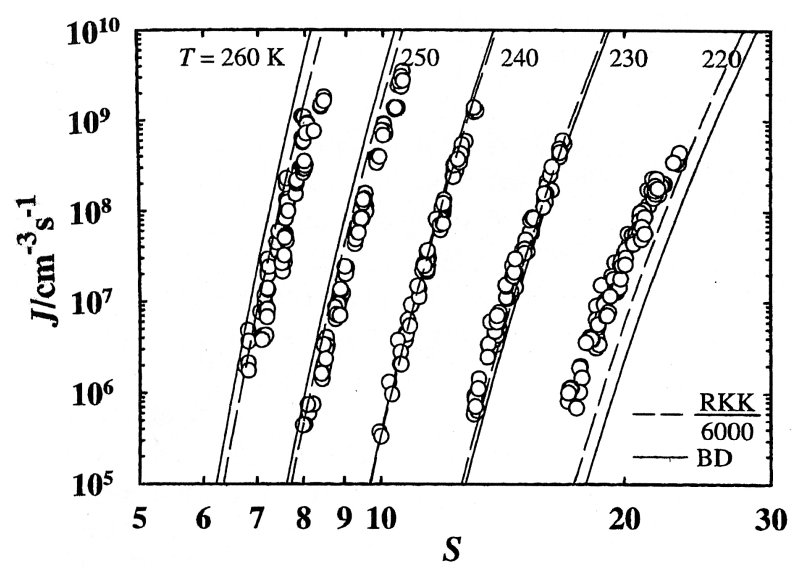

Fig. 4.7- $\mathrm{O}$ trabalho de RKK possibilitou a consistência interna da TCN, porém mesmo com o fator $6000 \mathrm{ad} h o c$, a dependência estranha com a temperatura persiste. No gráfico $S$ é a supersaturação. 


\section{5 \\ Teoria Entrópica da Nucleação}

\subsection{Introdução}

O fenômeno da nucleação é um processo intrinsecamente irreversível (visto no Cap. 2). A Teoria Entrópica da Nucleação (TEN) aborda o fenômeno analisando um processo reversível equivalente no qual há liberação de calor latente (variação da entalpia), concomitante a um rearranjo estrutural descrito pela variação da entropia antes e depois de certa quantidade de material ter nucleado. Para visualizar a dinâmica e facilitar a análise escolheu-se um processo isobárico, o qual é usualmente adotado em procedimentos experimentais [21][22].

\subsection{Fundamentação Teórica}

A existência de estados metaestáveis, que persistem por determinado intervalo de tempo, refletem o fato de que existem barreiras energéticas para a nucleação. Pequenas flutuações locais da fase atual (metaestável) tendem a ser absorvidas pelo sistema, enquanto que flutuações locais de maior amplitude podem romper vínculos internos e afetar todo o sistema. A formação de um núcleo da nova fase composto de $n$ partículas (ou monômeros) tal que $n \geq n^{*}$, deve-se àquela flutuação cuja amplitude levou uma pequena porção do sistema (elemento de volume) de determinado estado metaestável para um estado de instabilidade em relação à sua vizinhança. Não há dúvidas de que o processo de nucleação está vinculado à existência de flutuações, e que estas são manifestações do aspecto de muitos corpos de que são formados os sistemas termodinâmicos [17][19][10]. 
Uma vez ativado o processo, fala-se em taxa de nucleação $J$, eq. (1.1) ou seja, o número de núcleos por unidade de volume e de tempo

$$
J=J_{0} \exp \left(-\frac{\Delta G^{*}}{k_{B} T}\right)
$$

onde $J_{0}$ é um pré-fator associado a uma barreira cinética, $k_{B}$ é constante de Boltzmann, $T$ é a temperatura termodinâmica e $\Delta G^{*}$ é a barreira energética, ou seja, o trabalho mínimo necessário para a formação de um núcleo crítico da nova fase (no caso líquida). É natural portanto entender o trabalho crítico como proporcional ao número de elementos estruturais que formam o núcleo crítico, $n^{*}$, ou seja,

$$
\Delta G^{*} \propto n^{*}
$$

Conhecer a altura desta barreira de energia e/ou o tamanho do núcleo crítico é fundamental para a compreensão do processo de nucleação. É esperado que tal barreira energética diminua à medida que o estado metaestável aproxima-se do espinodal, quando então se anula, devido ao princípio de estabilidade termodinâmica, eq. (5.3). Equivale a dizer que nesse limite a transição de fase ocorrerá sem a necessidade de um núcleo, todo o sistema participa do processo.

A condição de estabilidade termodinâmica em forma local para um sistema fora do equilíbrio [20][10], é dada por

$$
\left(d^{2} s\right)_{e q}<0
$$

onde $s$ é a entropia específica e o sub-índice $e q$ indica que o desvio é a partir da posição de equilíbrio. Assumido que esta condição se verifica para o material que ao flutuar atinge a região de instabilidade e sofre transição de fase, pode-se dizer que a "força motriz" que "impulsiona" o processo de nucleação é a entropia. Considerando o processo como sendo isobárico (uma vez que a pressão ambiente não se altera durante o processo) a entropia em função das variáveis canônicas entalpia e pressão, apresenta-se como uma variável natural para estudar a nucleação (como visto no Cap. 2). 
Admitindo que a condição de estabilidade dada pela eq. (5.3) é válida também para um elemento de volume (cujo limite pode ser avaliado no Apêndice E), a estabilidade do sistema pode ser analisada a partir da variação da entropia específica como sendo função da entalpia específica e da pressão, $s=s(h, P)$. A primeira e segunda diferenciais são dadas por

$$
d s=\frac{1}{T} d h-\frac{v}{T} d P
$$

e

$$
d^{2} s=-\frac{1}{T^{2}}\left(\frac{\partial T}{\partial h}\right)_{P} d h^{2}-\left[\frac{1}{T}\left(\frac{\partial v}{\partial P}\right)_{T}-\frac{v}{T^{2}}\left(\frac{\partial T}{\partial P}\right)_{v}\right] d P^{2}
$$

ou,

$$
d^{2} s=-\frac{1}{T^{2} c_{p}} d h^{2}-\frac{v}{T}\left[\alpha-\frac{\beta}{T \alpha}\right] d P^{2},
$$

uma vez que $c_{p}=\left(\frac{\partial h}{\partial T}\right)_{P} \alpha=\frac{1}{v}\left(\frac{\partial v}{\partial T}\right)_{P}$ e $\beta=-\frac{1}{v}\left(\frac{\partial v}{\partial P}\right)_{T}$ são respectivamente coeficiente de expansão isobárica e coeficiente de compressão isotérmica.

A compreensão do limite de estabilidade termodinâmica está relacionada com o comportamento do calor específico do sistema que adentra a região metaestável (na Figura 5.8 o segmento de curva posterior ao ponto le para o líquido e o anterior ao ponto ge para o vapor). Da relação que conecta os calores específicos a pressão e volume constantes [17],

$$
c_{p}-c_{v}=-T \frac{\left(\frac{\partial P}{\partial T}\right)_{v}^{2}}{\left(\frac{\partial P}{\partial v}\right)_{T}}
$$

e da definição dos pontos no espinodal

$$
\left(\frac{\partial P}{\partial v}\right)_{T}=0
$$

verifica-se na eq. (5.7) que no espinodal $c_{p} \rightarrow \infty$, devido ao fato de $c_{p}>c_{v}$ - onde este último, por depender em primeira aproximação somente da temperatura, não diverge no espinodal — e de $\left(\frac{\partial P}{\partial T}\right)_{v}$ ser uma função que não diverge. 


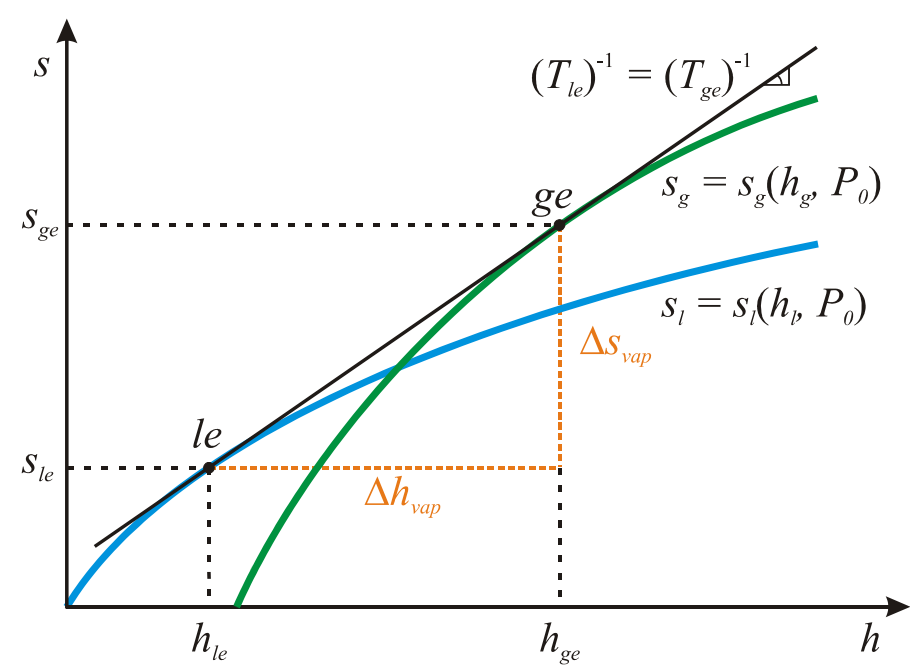

Fig. 5.8- O diagrama de Mollier foi modificado para tornar a entropia função da entalpia e também para evidenciar a região da metaestabilidade. $\Delta h_{v a p}$ e $\Delta s_{v a p}$ são o calor e a entropia de vaporização, respectivamente.

Como no espinodal $c_{p} \rightarrow \infty$, tem-se que à pressão constante a eq. (5.6) resulta

$$
\left(d^{2} s\right)_{P_{0}}=0
$$

Geometricamente, este ponto representa ponto de inflexão e, termodinamicamente, limite de estabilidade termodinâmica (conforme ilustrado pelos pontos $l s$ e $g s$ na Figura 5.9). Assim, para $d^{2} s<0$ o sistema encontra-se em estado estável ou metaestável; e para $d^{2} s>0$ implica em $c_{p}<0$, porém sem realidade física representando, portanto, instabilidade e inevitavelmente levando o sistema a sofrer transição de fase.

Nucleação é um processo irreversível e não existe transição de fase sem variação de entropia (produzida e/ou transferida). A entropia sendo uma diferencial exata permite certa flexibilidade na abordagem do processo de nucleação. Qualquer que seja o processo (isobárico, adiabático etc.) responsável pela nucleação de um pequeno elemento de volume, deve acarretar uma variação de entropia suficiente para produzir um núcleo estável na nova fase. Assim sendo, independentemente do processo e das condições de contorno sob as quais a nucleação se processa pode-se, para efeito de cálculo, escolher qualquer processo reversível, desde que as propriedades iniciais e finais do volume em evolução sejam as mesmas do processo real. A 
variação de entropia que o elemento de volume sofre ao evolver para a nova fase pode, portanto, ser calculada considerando o processo isobárico sem qualquer perda de generalidade [20].

Um fluido incompressível tem sua entropia específica em função da entalpia específica e da pressão expressa por

$$
s_{l}=c_{p, l} \ln \left[\frac{h-v_{l 3}\left(P_{0}-P_{3}\right)}{c_{p, l} T_{3}}+1\right] .
$$

onde admite-se que em estados estáveis da fase líquida os calores específicos equivalem-se, $c_{P}=c_{v}$, devido ao caráter incompressível do fluido [14] (vide Apêndice B) e o estado de referência é o ponto triplo da fase líquida.

E, analogamente, para um gás ideal,

$$
s_{g}=s_{v a p}+c_{P, g}^{\circ} \ln \left[\left(\frac{h-h_{v a p}}{c_{P, g}^{\circ} T_{3}}+1\right)\left(\frac{P_{3}}{P_{0}}\right)^{\frac{R}{c_{P, g}^{\circ}}}\right],
$$

onde $c_{P, g}^{\circ}$ é o calor específico a pressão constante de um gás ideal e $s_{v a p}$ e $h_{v a p}$ são a entropia e a entalpia específicos de vaporização no ponto triplo, respectivamente. Evidentemente que $s_{v a p}$ e $h_{v a p}$ dependem da pressão de vapor $P_{0}\left(\right.$ e de $\left.T_{e}=T\left(P_{0}\right)\right)$ (vide Apêndice B).

O diagrama de Mollier (entalpia-entropia) modificado $^{9}$ para evidenciar a transição de fase, conforme ilustrado na Figura 5.8, mostra de forma esquemática as duas fases — líquida e gasosa, descritas pelas equações (5.10) e (5.11), respectivamente — submetidas a uma pressão $P_{0}$ constante. A tangente comum às duas curvas (segmento de reta $\overline{l e g e}$ ) indica a linha de saturação. Os pontos le e ge representam o líquido e o vapor saturados, que tocam a tangente, em que se verifica

$$
\left(\frac{d s_{l}}{d h}\right)_{P_{0}, h=h l e}=\left(\frac{d s_{g}}{d h}\right)_{P_{0}, h=h g e}=\frac{1}{T_{e}}
$$

onde $T_{e}=T\left(P_{0}\right)$ é a temperatura de equilíbrio de fases. A diferença de entropia entre as fases no equilíbrio, bem como a de entalpia, fornece respectivamente a entropia e a entalpia latentes de vaporização, $\Delta s_{v a p}$ e $\Delta h_{v a p}$.

9 Por conveniência os eixos ordenados foram invertidos para facilitar análise da transição de fase (vide Sessão $6.1)$. 


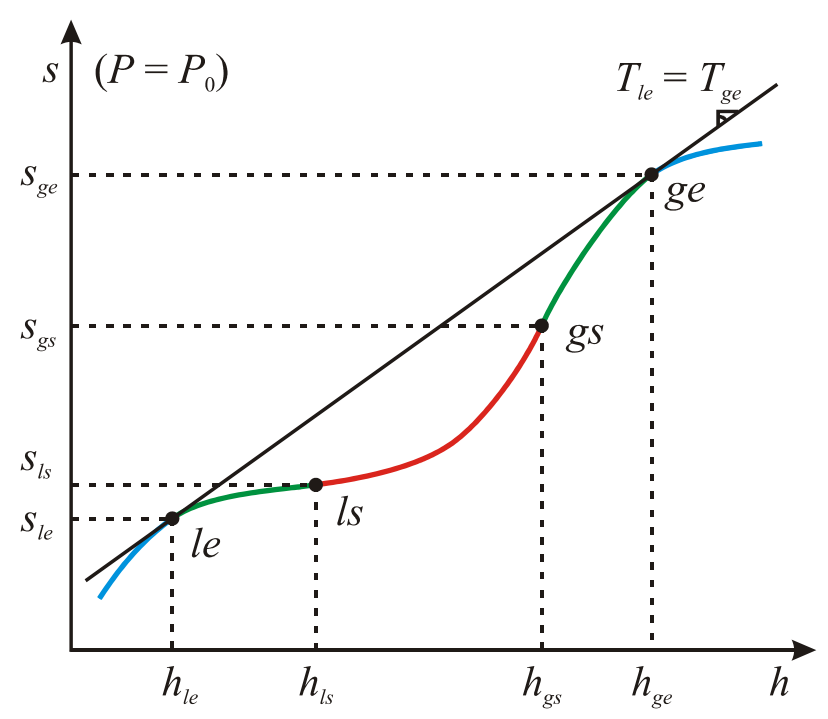

Fig. 5.9- A conexão entre as fases líquida e gasosa, devido à continuidade de estados, dá-se por uma curva suave que apresenta dois pontos de inflexão.

Entretanto, as eqs. (5.10) e (5.11) não trazem em sua estrutura o limite de estabilidade termodinâmica. São equações que descrevem suficientemente bem o fluido em estados estáveis longe do ponto crítico.

A análise da Figura 5.9 sugere que a transição de fase de primeira ordem, a partir de um estado metaestável, não pode ocorrer ao longo da tangente comum aos pontos de equilíbrio (le e ge na Figura 5.8). Isto é devido ao fato de a variação do volume não ser contínua, o que impossibilita uma variação linear da entalpia. Em conseqüência, a temperatura do elemento de volume deve variar.

Para uma função suave $s=\left(h, P_{0}\right)$ que, pela continuidade de estados, una a fase líquida à gasosa, nota-se na Figura 5.9 ser natural a existência do segmento de curva $\overline{l s g s}$ que conecta os pontos $l s$ e $g s$. Os segmentos posteriores a $l s$ e os anteriores a $g s$ representam regiões de metaestabilidade e estabilidade do sistema (descrita pelas equações (5.10) e (5.11)). Assim sendo, os pontos le e ge representam as duas fases em equilíbrio à mesma temperatura; os pontos $l s$ e $g s$ são, respectivamente, os espinodais da fase líquida e da fase gasosa. Os segmentos $\overline{l e l s}$ e $\overline{g s g e}$ da curva $s\left(h, P_{0}\right)$ representam regiões de metaestabilidade e, $\overline{l s g s}$ de instabilidade, onde ocorre a transição de fase (também conhecida como decomposição espinodal). 
A forma quadrática dada pela eq. (5.6) mostra que flutuações de $h$ e de $P$ são independentes, o que torna conveniente a escolha destas variáveis. A análise pode, portanto, proceder tomando $P=$ cte sem perda de generalidade, como já foi justificado anteriormente.

Cabe notar que os pontos $l e, l s$ e $g s, g e$ são encontrados tomando-se respectivamente as derivadas primeira e segunda da entropia em relação à entalpia a pressão constante, de modo que

$$
\left(\frac{\partial s}{\partial h}\right)_{P_{0}, h=h l e}=\left(\frac{\partial s}{\partial h}\right)_{P_{0}, h=h g e}=\frac{1}{T_{e}}
$$

e

$$
\left(\frac{\partial^{2} s}{\partial h^{2}}\right)_{P_{0}, h=h l s}=\left(\frac{\partial^{2} s}{\partial h^{2}}\right)_{P_{0}, h=h g s}=0 .
$$

Além disso, de forma geral a expressão $\left(\frac{\partial s}{\partial h}\right)_{P}=\frac{1}{T}$ indica a temperatura em qualquer ponto onde se verifica a estabilidade termodinâmica, eq. (5.3), evidenciando a conveniência do uso do diagrama de Mollier.

\subsection{Tamanho do Núcleo Crítico (Landau)}

As condições de estabilidade termodinâmica são válidas na região metaestável até o limite do espinodal (caracterizado pelos pontos $l s$ e $g s$ na Figura 5.9). Com o sistema em determinado estado metaestável, entre $g 9 u e$ e $g s$, o processo de nucleação inicia-se quando são verificadas duas condições: (i) um elemento de volume, constituído de $n$ moléculas, sofre flutuação de determinada magnitude de modo que o estado deste atinja ou ultrapasse o espinodal $g s$; (ii) na nova fase, o aglomerado de tamanho $n$ também flutua, porém seu estado não deve alcançar ou ultrapassar o espinodal $l s[20]$.

Seja um sistema constituído de $N$ monômeros de uma substância pura exibindo a fase vapor, submetido à pressão $P_{0}$ no estado de equilíbrio metaestável $\mathrm{gm}$. Então as propriedades termodinâmicas associadas ao seu estado metaestável são, entalpia $h_{g m}$, entropia $s_{g m}=s\left(h_{g m}, P_{0}\right)$ e temperatura $T_{g m}=T\left(h_{g m}, P_{0}\right)$. Tomando um elemento de volume com $n$ moléculas, tal que 
$n \ll N$, tem-se as mesmas propriedades termodinâmicas. Porém, nesta escala, as flutuações têm papel relevante. Interessa a flutuação que leva o estado do elemento de volume a atingir ou ultrapassar o espinodal da fase gasosa, $g s$. Estas flutuações podem ser caracterizadas quer pela entalpia, quer pela temperatura, conforme esquematizado na Figura 5.10.

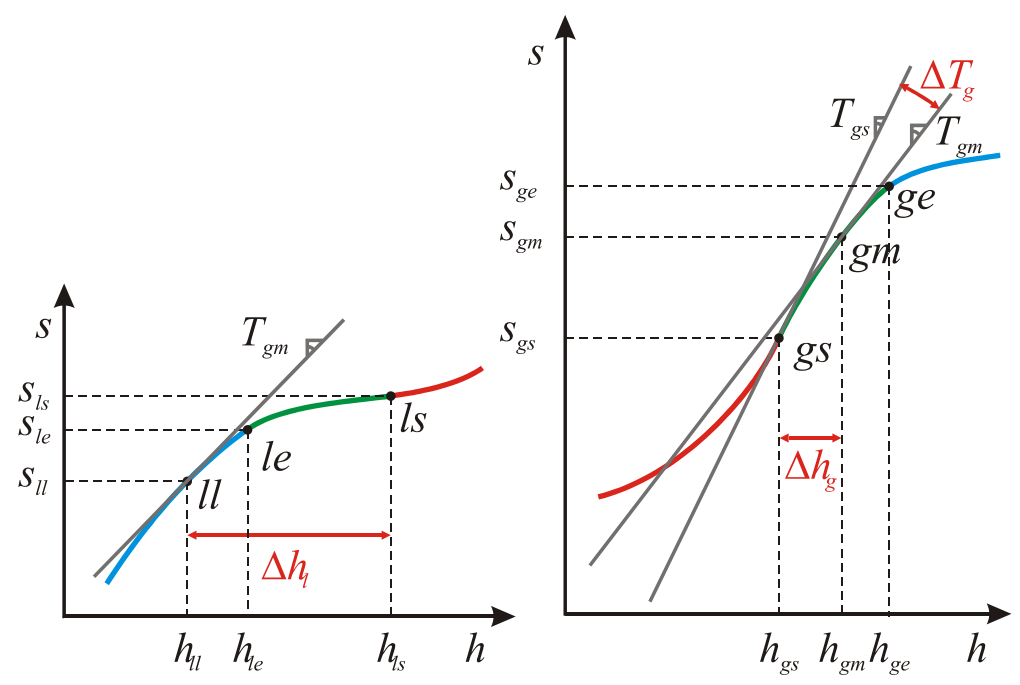

Fig. 5.10- Flutuação da temperatura e da entalpia da fase gasosa (direita) e flutuação da entalpia da fase líquida (esquerda).

A amplitude de flutuação da temperatura de um elemento de volume em torno do estado metaestável, gm (vide eq. (E.9) no Apêndice E), é dada por

$$
\left\langle\left(\Delta T_{g m}\right)^{2}\right\rangle^{1 / 2}=T_{g m} \sqrt{\frac{R}{n C_{v}}},
$$

onde $R$ é a constante dos gases ideais, $n$ é o número de partículas do elemento de volume envolvido.na flutuação e $C_{v}$ é a capacidade térmica molar a volume constante.

Se a amplitude de flutuação da temperatura é grande o bastante para carregar $n$ partículas para um estado que atinja ou ultrapasse o espinodal $g s$, (conforme ilustrado na Figura 5.10) é esperado que seja maior ou igual ao módulo da diferença de temperatura entre a do espinodal, $T_{g s}$, e a do estado metaestável, $T_{g m}$, ou seja,

$$
T_{g m} \sqrt{\frac{R}{n C_{v}}} \geq\left|T_{g s}-T_{g m}\right|
$$


ou, admitindo a igualdade, ser este o limite da fase metatestável, tem-se

$$
\frac{1}{\sqrt{n}} \sqrt{\frac{R}{C_{v}}}=\left|\frac{T_{g s}}{T_{g m}}-1\right|
$$

A correspondente flutuação da entalpia por partícula, em torno de $h_{g m}$ (vide eq. (E.20) no Apêndice E), é

$$
\left\langle\left(\Delta H_{g m}\right)^{2}\right\rangle^{1 / 2}=\lambda_{g} \sqrt{n}\left\langle h_{g m}\right\rangle
$$

onde $\lambda_{g}(>0)$ é uma constante de proporcionalidade, relacionada ao estado $g m$. Novamente, se a amplitude de flutuação da entalpia é grande o bastante para carregar $n$ partículas para um estado que atinja ou ultrapasse o espinodal $g s$, é esperado que seja maior ou igual ao módulo da diferença entre a entalpia do espinodal, $h_{g s}$, e a do estado metaestável, $h_{g m}$, ou seja,

$$
\lambda_{g} \sqrt{n} h_{g m} \geq n\left|h_{g s}-h_{g m}\right|
$$

ou, visto novamente ser suficiente o limite da igualdade, tem-se

$$
\frac{\lambda_{g}}{\sqrt{n}}=\left|\frac{h_{g s}}{h_{g m}}-1\right|
$$

onde considera-se $\left\langle h_{g m}\right\rangle \simeq h_{g m}$.

As eqs. (5.17) e (5.20), podem ser combinadas para eliminar $\sqrt{n}$ e isolar a constante de proporcionalidade, conforme Mokross [19] e Pasqua [23],

em que se verifica a não dependência explícita sobre $n$.

$$
\lambda_{g}=\sqrt{\frac{R}{C_{v}}} \frac{\left|\frac{h_{g s}}{h_{g m}}-1\right|}{\left|\frac{T_{g s}}{T_{g m}}-1\right|}
$$

Satisfeita a igualdade na eq. (5.21) o elemento de volume evolverá para a nova fase e flutuará em torno do estado estável $l l$, à temperatura $T_{l l}=T_{g m}$, e correspondente entalpia $h_{l l}$ e entropia $s_{l l}$. O fenômeno da nucleação ocorrerá se a amplitude de flutuação da entalpia da fase líquida não for capaz de fazer o estado do aglomerado atingir ou ultrapassar o espinodal do líquido $l s$; neste caso tem-se o rompimento da barreira cinética, desestabilizando o elemento de 
volume e, por conseqüência, o sistema. Portanto para o aglomerado da fase líquida não decair devido à flutuação deve-se verificar a seguinte inequação

$$
\lambda_{l} \sqrt{n} h_{l l}<n\left|h_{l s}-h_{l l}\right|
$$

ou,

$$
\sqrt{n}\left|\frac{h_{l s}}{h_{l l}}-1\right|>\lambda_{l}
$$

onde $\lambda_{l}(>0)$ é uma constante de proporcionalidade, relacionada ao estado $l l$ e $\left\langle h_{l l}\right\rangle \simeq h_{l l}$ é a entalpia por partícula.

O fator $\sqrt{R / C_{v}}$ na flutuação da temperatura em (5.15) tem papel de constante de proporcionalidade cujo valor refere-se ao estado $\mathrm{gm}$, assumindo o menor valor no espinodal [30]. A constante $\lambda_{g}$ na eq. (5.20) refere-se à fase gasosa metaestável no estado gm, obtida devido à flutuação da entalpia em torno de $h_{g m}$; e a constante $\lambda_{l}$ na eq. (5.23) refere-se à fase líquida estável no estado $l l$, devido à flutuação da entalpia em torno de $h_{l l}$, o qual tem a temperatura igual à do estado $g m$, ou seja, $T_{l l}=T_{g m}$. A igualdade das temperaturas evidencia o fato da amplitude das flutuações de cada fase, responsável pela formação de um núcleo, serem inversamente proporcionais. À medida que o estado metaestável aproxima-se do espinodal, diminui a amplitude de flutuação necessária para o elemento de volume sofrer transição de fase; contrariamente, aumenta a amplitude de flutuação da fase líquida necessária para decair o aglomerado. Evidentemente que a pressão constante $P_{0}$ está subentendida em todo o cenário. Diante disso, é natural pensar que as constantes de proporcionalidade, $\lambda_{g}$ e $\lambda_{l}$ são proporcionais, ou seja,

$$
\lambda_{l}=\sqrt{\phi} \lambda_{g}
$$

onde $\phi$ é uma constante a ser definida [23]. Isto permite relacionar as eqs. (5.20) e (5.23) da forma

$$
\sqrt{n}\left|\frac{h_{l s}}{h_{l l}}-1\right|>\lambda_{l}=\sqrt{\phi} \sqrt{\frac{R}{C_{v}}} \frac{\left|\frac{h_{g s}}{h_{g m}}-1\right|}{\left|\frac{T_{g s}}{T_{g m}}-1\right|}
$$


de modo que é possível elinar $\lambda_{l}$ e fazer $n \equiv n^{*}$ assumindo a condição mínima (no limite da igualdade), ou seja,

$$
n^{*}=\phi \frac{R}{C_{v}} \frac{\left(\frac{h_{g s}}{h_{g m}}-1\right)^{2}}{\left(\frac{T_{g s}}{T_{g m}}-1\right)^{2}\left(\frac{h_{l s}}{h_{l l}}-1\right)^{2}}
$$

Segundo Mokross [19], esta proporcionalidade significa que maior metaestabilidade está correlacionada com maior intensidade de nucleação.

Cabe notar que no cenário da TEN, a eq. (5.26) tem como variáveis a pressão e a entalpia, doravante assumida ser específica, sem comprometimento da adimensionalidade da constante $\phi$. Assim,

$$
\begin{gathered}
T_{g m}=T\left(h_{g m}, P_{0}\right)=\left[1 /\left(\frac{d s(h, P)}{d h}\right)_{P=P_{0}}\right]_{h=h_{g m}} \\
h_{l l}=\operatorname{Roots} O f\left[\left(\frac{\partial s(h, P)}{\partial h}\right)_{P=P_{0}}=\frac{1}{T_{g m}}, h<h_{l e}\right]
\end{gathered}
$$

e é esperado escrever $C_{v}$ em termos específicos como

$$
c_{v}=c_{p}\left[1-P_{0}\left(\frac{\partial v}{\partial h}\right)_{P=P_{0}}\right]
$$

onde

$$
v=v(h, P)=\left(\frac{\partial h}{\partial P}\right)_{s}
$$

sendo $v$ volume específico (vide Apêndice E).

O número de monômeros necessários para formar um núcleo crítico dado pela eq. (5.26) é válido se a nucleação for homogênea (modelo de Szilard) [19]. Porém a eq. (5.26) está incompleta no sentido que parâmetros de superfície não foram considerados. Flutuações como mencionadas acima formam aglomerados de diversos tamanhos: os núcleos estabilizam-se, enquanto que os embriões decaem. Além disso, a teoria da flutuação de Landau diz respeito a pequenas flutuações em torno do ponto médio. Entende-se que estes resultados preliminares, obtidos através da teoria da flutuação de termodinâmica de equilíbrio (Landau), quando aplicada a sistemas pequenos não pode dar resultados seguros, visto que próximo do espinodal 
as flutuações de energia são grandes [30]. Além do mais, sistemas pequenos estão longe do limite termodinâmico e parâmetros intrínsecos não estão bem definidos (vide Apêndice E). É necessário uma teoria de flutuação que considere flutuações de qualquer magnitude e possa lidar com aglomerados e núcleos em uma abordagem termodinâmica.

\subsection{Cálculo do Núcleo Crítico}

A TEN fez uso de flutuações sobre o diagrama de Mollier para desenvolver a expressão do núcleo crítico. Nesse cenário, é desejável uma função entropia, $s(h, P)$ que forneça dados da região metaestável. Tal função foi obtida conforme mostrado no Capítulo 6. Porém os cálculos preliminares do tamanho do núcleo crítico via TEN (flutuação de Landau) foram realizados inicialmente com ajuda da equação de estado de van der Waals (EE-vdW) [23] e, em seguida, com a equação de estado de Peng-Robinson (EE-PR), dada por

$$
P=\frac{R T}{v-b}-\frac{a(T, \omega)}{v^{2}+2 b v-b^{2}}
$$

onde o fator relacionado às forças de coesão $a(T, \omega)$ é função da temperatura e do fator acéntrico $\omega$, conforme Apêndice A.2.

$\mathrm{Na}$ eq. (5.26) a capacidade térmica molar a volume constante foi escrita como $C_{v}=$ $3 R / 2$, aproximação válida para estados próximos do ponto triplo, quando o vapor de água apresenta características de gás ideal (as experiências de nucleação foram realizadas nessa região, conforme Apêndice F). No entanto, é forçado tratar $C_{v}$ como constante na região metaestável. Assumindo a igualdade na expressão para o núcleo crítico, eq. (5.26), $C_{v}=3 R / 2$ e fazendo $\phi=1$, tem-se

$$
n^{*}=\frac{2}{3}\left[\frac{\left(\frac{h_{g s}}{h_{g m}}-1\right)}{\left(\frac{T_{g s}\left(h_{g s}\right)}{T_{g m}\left(h_{g m}\right)}-1\right)\left(1-\frac{h_{l s}}{h_{l l}\left(T_{g m}\right)}\right)}\right]^{2}
$$


Tal expressão exige o conhecimento da temperatura e da entalpia nos pontos metaestável ( $T_{g m}$ e $\left.h_{g m}\right)$, no espinodais $\left(T_{g s}, h_{g s}\right.$ e $\left.h_{l s}\right)$ e no líquido estável $\left(h_{l l}\right)$ para obter o tamanho do núcleo crítico.

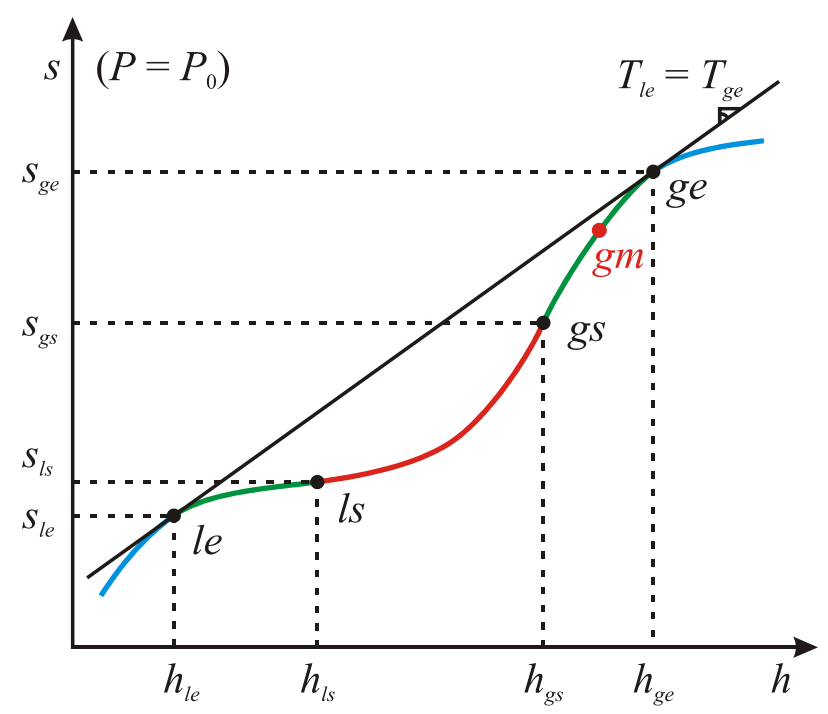

Fig. 5.11- No diagrama de Mollier, o ponto gm é o ponto do vapor no estado metaestável.

Na Figura 5.11 representou-se o estado metaestável $\mathrm{gm}$ em um diagrama de Mollier, juntamente com os pontos necessários para o cálculo do núcleo crítico. Na ausência da função entropia $s=s(h, P)$ para a região metaestável, optou-se por calcular o tamanho do núcleo crítico $n^{*}$ fazendo uso de um diagrama $P, v$ para encontrar as grandezas volume e temperatura. Para o cálculo da entalpia nos espinodais, usou-se as Funções Residuais [32] [23]. Tais funções permitem o cálculo do desvio nas propriedades termodinâmicas de uma substância real em comparação com uma substância ideal. Para a entalpia, tal função é da forma

$$
h(T)=h_{g 3}+c_{v}^{\circ}\left(T-T_{3}\right)+h_{r e s}
$$

onde $h_{g 3}$ é a entalpia de vaporização da água no ponto triplo, $c_{v}^{\circ}=c_{p}^{\circ}-R$ é o calor específico a volume constante do gás ideal e $h_{\text {res }}$ é a função residual. Esta tem a forma geral dada por

$$
h_{r e s}=-\int_{\infty}^{v_{f}}\left[v\left(\frac{\partial P}{\partial v}\right)_{T}+T\left(\frac{\partial P}{\partial T}\right)_{v}\right]_{T_{f}} d v
$$


Para tanto, é assumido que

1. a temperatura do elemento de volume não é constante durante a transição de fase, ainda que macroscopicamente a temperatura seja a mesma antes e depois de verificado o processo.

2. o calor específico a volume constante mantém-se constante na região metaestável, mesmo no espinodal.

3. a EE-vdW e EE-PR e por extensão Funções Residuais, são válidas na região metaestável até o espinodal

4. a EE-vdW e EE-PR e por extensão Funções Residuais, são válidas para um elemento de volume (vide Apêndice E)

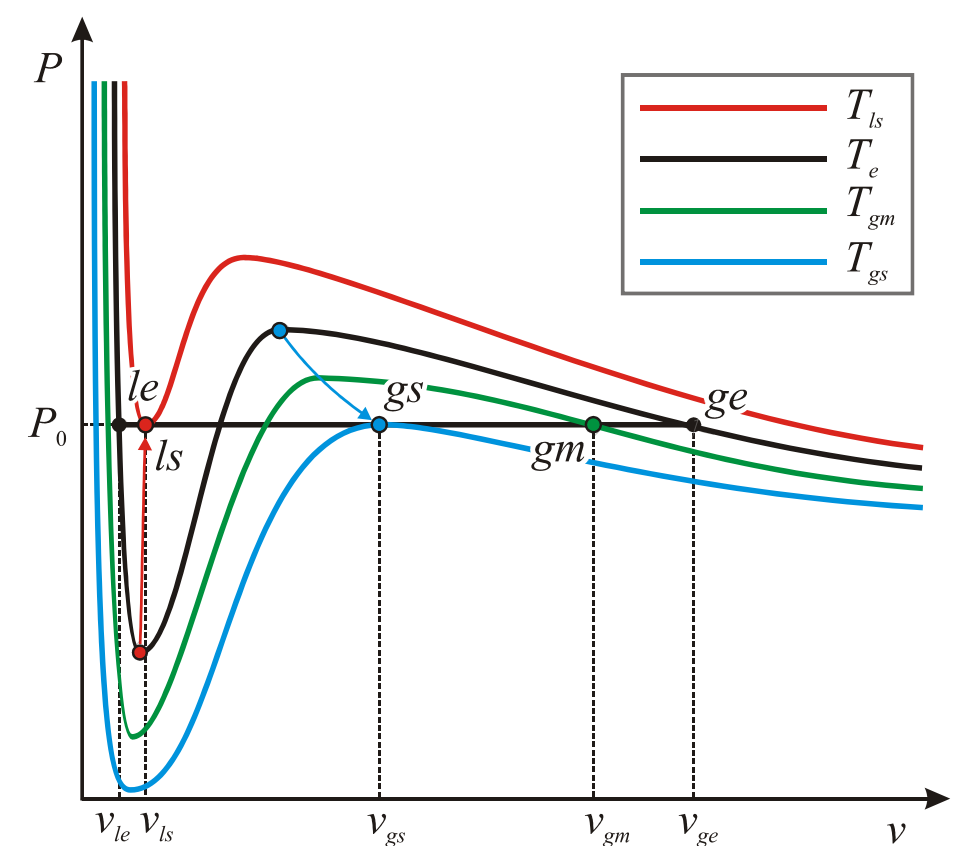

Fig. 5.12- Os pontos binodais, espinodais e metaestável estão sobre a reta $P=P_{e}$.

Os dados experimentais do estado metaestável $\mathrm{gm}$, a dizer, pressão e temperatura, $P_{\exp }$ e $T_{\exp }$, foram obtidos a partir dos trabalhos de Viisanen et al. [33] e de Wölk-Strey [36] (vide Apêndice F). Com ajuda da eq. (5.31), o equivalente estado metaestável é representado pelo 
ponto $g m$ na Figura 5.12. Este ponto corresponde à temperatura, $T_{\exp }$, e pressão, $P_{\exp }$. Na Figura 5.12, o ponto $g m$ está localizado sobre a reta que une o estado gasoso, ge, e o líquido, $l e$, que corresponde à isoterma de equilíbrio cuja pressão e temperatura designou-se por $P_{0} \mathrm{e}$ $T_{e}=T\left(P_{0}\right)$, respectivamente.

No processo de nucleação proposto, o ponto $g m$ que representa o estado metaestável tem uma temperatura $T_{g m}=T_{\exp }$ que é diferente da temperatura $T_{e}$ de equilíbrio de fases, conforme representado na Figura 5.12, pois $T_{\exp }$ está no loop de van der Waals que representa o elemento de volume que sofre flutuação. A pressão $P_{\text {exp }}$ é igual à pressão $P_{0}$, visto que o processo assumido se realiza a pressão constante. Pode-se escrever

$$
P_{\exp } \equiv P_{0}, \quad T_{\exp } \equiv T_{g m}
$$

Segundo este mecanismo de nucleação, o elemento de volume sofrerá uma flutuação que o levará para além do espinodal $g s$, atingindo a região de instabilidade (nas Figuras 5.11 e 5.12 representado pelo segmento de curva $\overline{l s g s}$ ), sofrerá transição de fase e formará o aglomerado da fase nova no estado $l l$, caracterizado pela temperatura $T_{l l}=T_{g m}$. Se o sistema inteiro, depois da transição de fase total, retorna à temperatura de equilíbrio $T_{l e}=T_{g e}=T_{e}$, é irrelevante uma vez que o foco desta análise é a formação de um núcleo crítico.

\subsubsection{Algoritmo}

A expressão para o núcleo crítico, eq. (5.32), requer o cálculo das grandezas temperatura e entalpia no metaestável $\left(T_{g m}\right.$ e $\left.h_{g m}\right)$, no espinodal $\left(T_{g s}, h_{g s}\right.$ e $\left.h_{l s}\right)$ e no líquido estável $\left(h_{l l}\right)$, cuja determinação foi efetuada da maneira exposta a seguir.

Sendo $P_{\exp }=P_{0}$, encontra-se $T_{e}$, por exemplo, pela equação empírica de P. E. Wagner ${ }^{10}$

$$
P_{0}=\exp \left[77.34491-\frac{7235.42465}{T_{e}}-8.2 \ln \left(T_{e}\right)+0.0057113 T_{e}\right]
$$

10 WAGNER, P. E. Areosol Research III, Wien, 209, 1981; apud Wölk-Strey [36]. 
Com $P_{0}$ e $T_{e}$ na equação (5.31) resolvida para $v$ encontra-se $v_{l e}$ e $v_{g e}$, de modo que se tem os pontos binodais $g e=\left(P_{0}, v_{g e}, T_{e}\right)$ e $l e=\left(P_{0}, v_{l e}, T_{e}\right)$.

Com $P_{\exp }=P_{0}$ e $T_{\exp }=T_{g m}$ na equação (5.31), encontra-se $v_{g m}$ e $v_{l l}$ Tem-se assim, o ponto metaestável, $g m=\left(P_{0}, v_{g m}, T_{g m}\right)$ e o ponto estável, $l l=\left(P_{0}, v_{l l}, T_{g m}\right)$.

Os pontos espinodais para a fase gasosa e para a fase líquida são encontrados conforme definição dada pela equação (5.8) calculada sobre a equação (5.31). Resulta uma função que depende de $v$ e de $T$, a qual pode ser resolvida para a temperatura no espinodal, $T_{s}=T\left(v_{s}\right)$. onde $T_{s}$ e $v_{s}$ são respectivamente temperatura e volume do fluido no espinodal $\left(v_{l s}\right.$ e $\left.v_{g s}\right)$, ainda desconhecidos. Levando $T_{s}$ na equação (5.31) tem-se a pressão em função do volume do espinodal, $P_{s}=P\left(v_{s}\right)$. Impondo que $P_{s}=P_{0}$ obtém-se os volumes dos espinodais caracterizados por $v_{l e}<v_{l s}<v_{c}$ e $v_{c}<v_{g s}<v_{g m}$, os quais levados em $T_{s}$ resulta as respectivas temperaturas nos espinodais, $T_{l s}=T_{s}\left(v_{l s}\right)$ e $T_{l s}=T_{s}\left(v_{l s}\right)$. Tem-se então os pontos espinodais $g s=\left(P_{0}, v_{g s}, T_{g s}\right)$ e $l s=\left(P_{0}, v_{l s}, T_{l s}\right)$.

A Figura 5.13 mostra o tamanho do núcleo crítico em função da temperatura, em que se comparam os dados experimentais com os cálculos do núcleo crítico usando a eq. (5.32) combinada com a EE-vdW e com a EE-PR. Os núcleos experimentais, $n_{\exp }^{*}$ foram obtidos pelo Método dos Mínimos Quadrados aplicados a uma função linear sobre um conjunto de dados da taxa de nucleação $J$ em relação à supersaturação $S_{s}$, para diferentes configurações do sistema [23], como pressão, temperatura e fração molar. Um resumo desses dados encontrase no Apêndice F. Independentemente do modelo teórico, a variação do logaritmo da taxa de nucleação em relação ao logaritmo da supersaturação, tomados a temperatura constante é comparada ao tamanho do núcleo crítico, ou seja,

$$
\left(\frac{\partial \ln J}{\partial \ln S_{s}}\right)_{T}=n^{*}
$$

Esse é o núcleo crítico experimental. 
O comportamento da eq. (5.32), em que a constante de proporcionalidade foi assumida unitária, $\phi=1$, e a capacidade térmica molar a volume constante foi considerada constante na região metaestável, é similar ao experimental. Isto é indicativo de que flutuações, ainda que em formulação mais simples (Landau) podem auxiliar na descrição do fenômeno da nucleação. Nota-se que a comparação é feita mediante fatores ad hoc da ordem de $10^{3}$. Essa mesma ordem de grandeza é encontrada, mais adiante no Capítulo 6, em que se comparou o núcleo crítico obtido através da eq. (5.32) com a teoria de Dillmann-Meier [4][5].
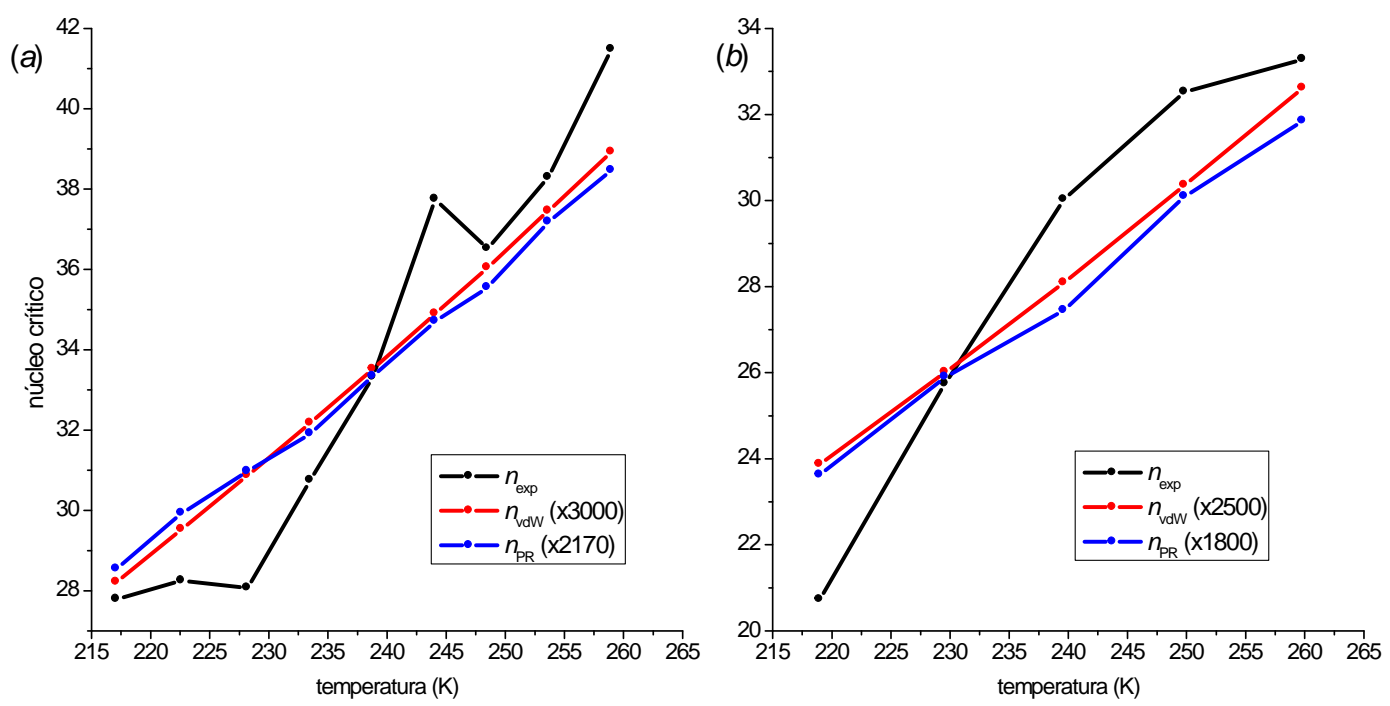

Fig. 5.13- Núcleo crítico em função da temperatura. À esquerda a experiência de Viisanen et al. e à direita a experiência de Wölk-Strey. Em ambas, em preto é o núcleo crítico calculado pelo Método dos Mínimos Quadrados, em vermelho, com auxílio da EE-vdW, e em azul com ajuda da EE-PR.

Apesar dos resultados preliminares serem qualitativamente satisfatórios, tem-se que as EE-vdW e a EE-PR não são adequadas para o estudo da região metaestável, são equações inicialmente destinadas à descrição de estados do fluido na fase gasosa. A fase líquida estudada a partir destas equações, apresenta desvios consideráveis como mostrado no Apêndice A.2. As temperaturas calculadas no espinodal do líquido são da ordem de $10^{0}$ o que não faz sentido físico; a flutuação da temperatura a partir do estado $\mathrm{gm}$ resultaria magnitude da ordem de $10^{2}$. 
Conclui-se que é desejável uma função entropia que represente a região metaestável de forma mais realista. 


\section{6 \\ Função Entropia}

\subsection{Diagrama de Mollier}

Richard Mollier (1863-1935) foi o primeiro a reconhecer as vantagens de se usar a entalpia como coordenada nos diagramas de estado. À época da primeira publicação do diagrama entalpia-entropia para o vapor de água (1904) era professor na Universidade de Dresden (Alemanha). No diagrama $h, s$ da Figura 6.14, a linha de saturação separa a zona de condensação da zona de vapor superaquecido ${ }^{11}$. Dentro da zona de condensação as isóbaras confundem-se com as isotermas e a região de coexistência. Equivale a dizer que se a condensação se dá a pressão constante, a temperatura também será constante. Além do mais, se a mudança de estado se dá sem dissipação, será uma isentrópica (reta vertical). Na zona de vapor superaquecido, as isóbaras encontram-se separadas das isotermas.

Além de se obter entalpia e entropia diretamente de um ponto sobre o diagrama, também é possível de se obter facilmente a temperatura, a pressão, o volume (alguns diagramas apresentam isócoras). Também é possível de se obter o calor específico a pressão constante, na zona de vapor superaquecido a pendente da isóbara está relacionada com o calor específico $c_{P}=(d h / d T)_{P} ;$ de modo similar é possível encontrar $c_{v}$ caso o diagrama apresente isócoras. No diagrama $h, s$ a distância vertical entre os estados inicial e final $(\Delta h)$ é a medida da realização do trabalho, e a distância horizontal $(\Delta s)$ é a medida das irreversibilidades associadas ao processo.

É sabido que todo gráfico representa o conjunto solução de uma função e que aspectos geométricos refletem propriedades físicas. Desse modo é esperado que uma função $s\left(h, P_{0}\right)$ que

11 KEENAN, J. H. and KEYES, F. G. Properties of Steam. John Wiley \& Sons, Inc; apud Kestin [14], 


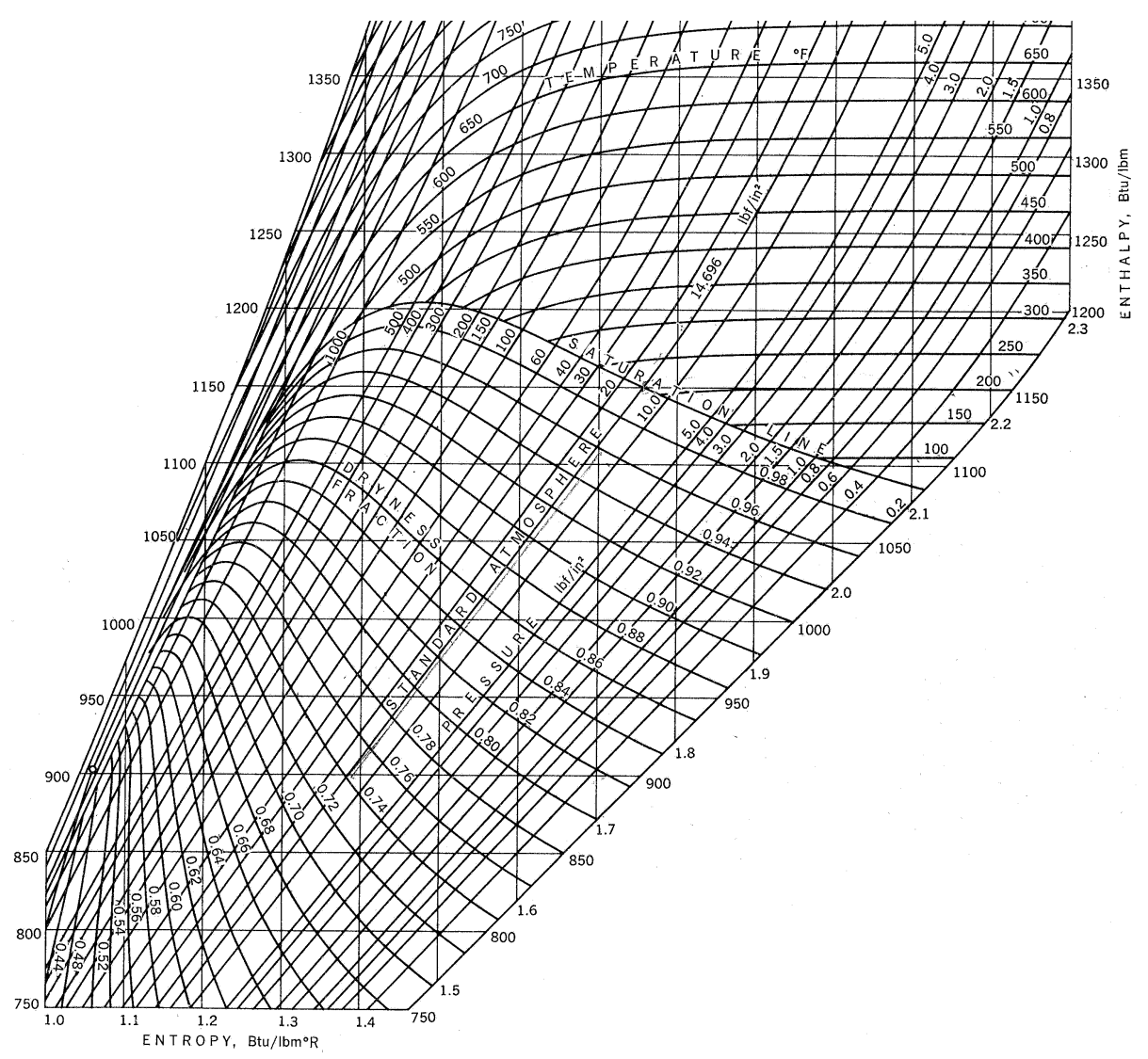

Fig. 6.14- Diagrama de Mollier da água.

adentre o estado metaestável até o limite do espinodal deve apresentar um ponto de inflexão como limite de estabilidade termodinâmica da fase (como mostrado na Sessão 5.2). As funções obtidas de líquido incompressível e gás ideal (Apêndice B) não conseguem representar o espinodal. Obter uma função $s\left(h, P_{0}\right)$ que una suavemente os pontos le e ge exige o conhecimento de dados e/ou propriedades da região metaestável do líquido e do vapor.

\subsection{Sobre o polinômio interpolador}

Existem muitos dados experimentais para água pura em vasta extensão da fase líquida estável (ll) e vapor estável $(g g)$; também há dados do fluido saturado (le e ge). Porém são escassos os dados experimentais na região metaestável $(l m$ e $g m)$. Com os dados experimentais disponíveis o International Association for the Properties of Water and Steam (IAPWS) [34][35] elaborou 


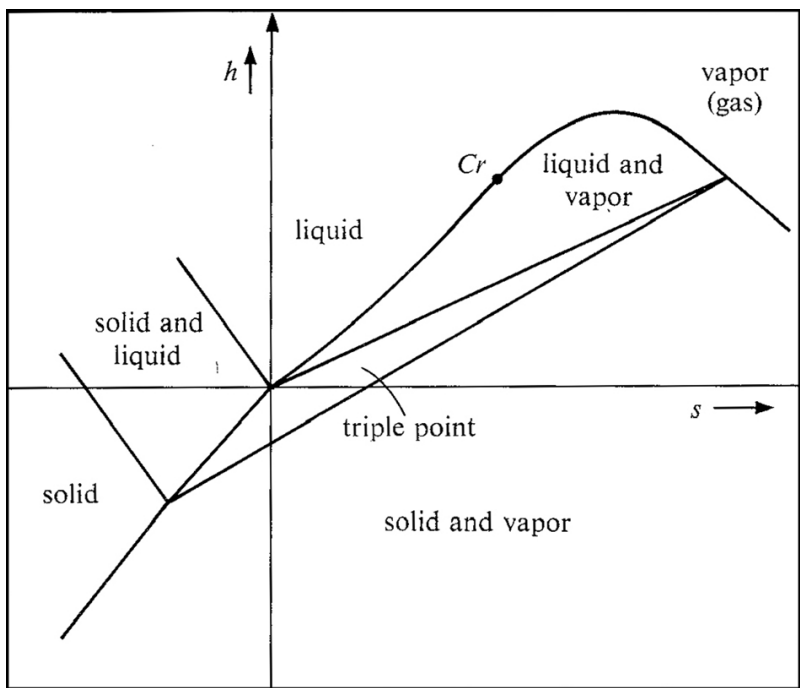

Fig. 6.15- Diagrama de Mollier estilizado, em que se evidenciam as fases de um fluido puro (conf. Kestin [14]).

um algoritmo que fornece dados com acurária e consistência ao longo de regiões estáveis do fluido. Na região do líquido a incerteza estimada da densidade varia entre $\pm 0.001 \%$ a $\pm 0.02 \%$ e da capacidade térmica a pressão constante $\pm 0.1 \%$. Em grande parte da região do gás a incerteza da densidade encontra-se entre $\pm 0.03 \% \mathrm{a} \pm 0.05 \%$, enquanto que a da capacidade térmica a pressão constante é $\pm 0.2 \%$. Entretanto, o algoritmo da IAPWS fornece pontos da região metaestável do líquido apenas em torno do ponto de equilíbrio de fases para o líquido e $5 \%$ da mistura da entalpia saturada para o vapor $\left[h_{r l e}-0.95\left(h_{r g e}-h_{r l e}\right)\right]$. Segundo a IAPWS não há dados do vapor de água no estado metaestável.

Na ausência de uma função de estado $s(h, P)$ que una suavemente os pontos le e ge, optou-se por uma função polinomial obtida por interpolação. Em cada ponto de estados estáveis e metaestáveis a função deve ter as seguintes propriedades

$$
\begin{gathered}
\left(\frac{\partial s}{\partial h}\right)_{P}=\frac{1}{T} \\
\left(\frac{\partial^{2} s}{\partial h^{2}}\right)_{P}=-\frac{1}{T^{2} c_{p}}
\end{gathered}
$$

em especial nos binodais 


$$
\begin{gathered}
\left(\frac{\partial s}{\partial h}\right)_{P, h=h l e}=\left(\frac{\partial s}{\partial h}\right)_{P, h=h g e}=\frac{1}{T_{e}} \\
\left(\frac{\partial^{2} s}{\partial h^{2}}\right)_{P, h=h l e}=-\frac{1}{T_{e}^{2} c_{p, l e}}\left(\frac{\partial^{2} s}{\partial h^{2}}\right)_{P, h=h g e}=-\frac{1}{T_{e}^{2} c_{p, g e}}
\end{gathered}
$$

e nos espinodais (limite da estabilidade termodinâmica)

$$
\begin{gathered}
\left(\frac{\partial s}{\partial h}\right)_{P, h=h l s}=\frac{1}{T_{l s}}\left(\frac{\partial s}{\partial h}\right)_{P, h=h g s}=\frac{1}{T_{g s}} \\
\left(\frac{\partial^{2} s}{\partial h^{2}}\right)_{P, h=h l s}=\left(\frac{\partial^{2} s}{\partial h^{2}}\right)_{P, h=h g s}=0
\end{gathered}
$$

Uma outra importante propriedade é a verificação da construção de Maxwell, ou seja,

$$
\oint d s\left(h, P_{0}\right)=\int_{l e}^{i n}\left[\frac{1}{T_{e}}-\left(\frac{\partial s}{\partial h}\right)_{P}\right] d h+\int_{i n}^{g e}\left[\left(\frac{\partial s}{\partial h}\right)_{P}-\frac{1}{T_{e}}\right] d h=0
$$

onde in é um estado instável do fluido que possui a seguinte propriedade matemática

$$
\left(\frac{\partial s}{\partial h}\right)_{P, h=h_{i n}}=\frac{1}{T_{e}}
$$

Visto a entalpia e a pressão serem grandezas termodinâmicas independentes, como mostrado nas eq. (5.4) e (5.6) propõe-se a função polinomial

$$
s(h, P)=\sum_{i=0}^{m} \sum_{j=0}^{n} P^{i} a_{i j} h^{j}
$$

onde $a_{i j}$ são coeficientes. Fixando a pressão $P=P_{0}$ pode-se escrever

$$
s\left(h, P_{0}\right)=\sum_{j=0}^{n} b_{j} h^{j}
$$

onde

$$
b_{j}=b_{j}\left(P_{0}\right)=\sum_{i=0}^{m} P_{0}^{i} a_{i j}
$$

Para a interpolação, os desconhecidos espinodais devem ser usados, visto sua importância física e geométrica. Isto significa que além das incógnitas $b_{0}, b_{1}, \ldots, b_{n}$ do sistema $\Omega_{I}$, eq. (6.12), existem as ordenadas desconhecidas $h_{l s}, s_{l s}, h_{g s}$ e $s_{g s}$. A solução encontrada foi construir outros 
dois sistemas de equações, $\Omega_{I I}$ e $\Omega_{I I I}$, baseados na derivada parcial primeira e segunda de (6.10) em relação a $h$. A $i$-ésima linha de cada sistema é dada por

$$
\begin{gathered}
\Omega_{I}: \quad \sum_{j=0}^{n} h_{i}^{j} \cdot b_{j}^{(I)}=s_{i} \\
\Omega_{I I}: \quad \sum_{j=1}^{n} j \cdot h_{i}^{j-1} \cdot b_{j}^{(I I)}=\frac{1}{T_{i}}
\end{gathered}
$$

e

$$
\Omega_{I I I}: \quad \sum_{j=2}^{n}(j-1) \cdot j \cdot h_{i}^{j-2} \cdot b_{j}^{(I I I)}=-\frac{1}{T_{i}^{2} c_{p, i}}
$$

onde, por construção, são iguais os coeficientes dos três sistemas,

$$
b_{j}^{(I)}=b_{j}^{(I I)}=b_{j}^{(I I I)}=b_{j}
$$

Entretanto, é conveniente identificá-los em cada sistema. Como será mostrado adiante, combinando os três sistemas em um quarto sistema é possível eliminar os coeficientes $b_{0}, b_{1}, \ldots, b_{n}$, e encontrar as incógnitas $h_{l s}, s_{l s}, T_{l s}, h_{g s}, s_{g s}$.e $T_{g s}$, impondo vínculos físicos e geométricos.

No Apêndice D é apresentado uma solução alternativa da que será apresentada a seguir. Por razões geométricas e algébricas, usou-se 9 pontos para construir um polinômio de grau 9 , capaz de representar a região de continuidade dos fluidos, regiões metaestável líquido, região de instabilidade e região metaestável do gás (vide Figura 6.16). Desses nove pontos, 5 pertencem à fase líquida da água $\left(l l_{1}, l l, l e, l m, l s\right)$. O ponto $l l_{1}$ é usado para tornar implícito os coeficientes $b_{0}$ e $b_{1}$. Os pontos não-alinhados $l l_{1}, l l$, le e $l m$ formam a curvatura da fase líquida das funções $s\left(h, P_{0}\right),(\partial s / \partial h)_{P_{0}}$ e $\left(\partial^{2} s / \partial h^{2}\right)_{P_{0}}$ em torno do binodal le que geometricamente é importante para indicar a região onde deve estar o espinodal $l s$. Os outros quatro pontos $g s, g m, g e$ e $g g$ pertencem à fase gasosa. Os pontos $g m, g e$ e $g g$ fornecem a curvatura em torno de $g e$ que deverá induzir o ponto espinodal $g s$. 


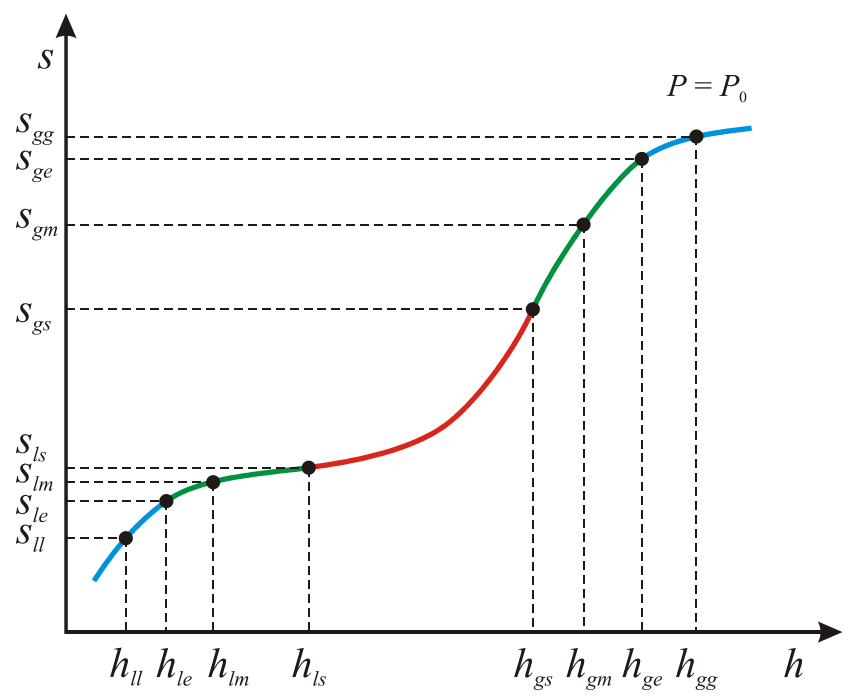

Fig. 6.16- Os subíndices $l l, l e, l m, l s, g s, g m, g e, g g$ representam os pontos do líquido nos estados estável, ETD, metaestável, espinodal e do gás nos estados espinodal, metaestável, ETD, estável, respectivamente.

Para que os três sistemas tenham a mesma dimensão a $i$-ésima linha de cada um é escrita como

$$
\begin{aligned}
& \Omega_{I}: \quad \sum_{j=2}^{9} h_{i}^{j} \cdot b_{j}^{(I)}=s_{i}-b_{1} h-b_{0} \\
& \Omega_{I I}: \quad \sum_{j=2}^{9} j \cdot h_{i}^{j-1} \cdot b_{j}^{(I I)}=\frac{1}{T_{i}}-b_{1}
\end{aligned}
$$

e

$$
\Omega_{I I I}: \quad \sum_{j=2}^{9}(j-1) \cdot j \cdot h_{i}^{j-2} \cdot b_{j}^{(I I I)}=-\frac{1}{T_{i}^{2} c_{p, i}}
$$

O supraíndice de $b_{0}$ e de $b_{1}$ foi omitido para que com o auxílio do ponto $l l_{1}$ venham a se tornar implícitos nas equações (6.16) e (6.17) Isso pode ser feito devido à equação (6.15).

Fazendo $h_{i}=h_{l l 1}$ e $T_{i}=T_{l l 1}$ em (6.17) e isolando $b_{1}$ resulta

$$
b_{1}=\frac{1}{T_{l l_{1}}}-\sum_{j=2}^{9} j \cdot h_{l l_{1}}^{j-1} \cdot b_{j}^{(I)}
$$

onde a troca do supraíndice do coeficiente $b_{j}^{(I I)}=b_{j}^{(I)}$ é conveniente para tornar implícito $b_{1}$ no sistema $\Omega_{I}$. Fazendo $h_{i}=h_{l l 1}$ e $s_{i}=s_{l l 1}$ em (6.16) e usando $b_{1}$ encontrado acima resulta

$$
b_{0}=s_{l l_{1}}-\frac{h_{l l_{1}}}{T_{l l_{1}}}+\sum_{j=2}^{9}(j-1) \cdot h_{l l_{1}}^{j} \cdot b_{j}^{(I)}
$$


Substituindo as equações (6.19) e (6.20) em (6.16), a $i$-ésima linha do sistema $\Omega_{I}$ quadrático de dimensão 8, é escrita como

$$
\Omega_{I}: \quad \sum_{j=1}^{8}\left[h_{i}^{j+1}-(j+1) h_{i} h_{l l_{1}}^{j}+j h_{l l_{1}}^{j+1}\right] b_{j+1}^{(I)}=\left(s_{i}-s_{l l_{1}}\right)-\frac{\left(h_{i}-h_{l l_{1}}\right)}{T_{l l_{1}}}
$$

onde houve renomeação do subíndice $j \rightarrow j+1$. A $i$-ésima linha dos sistemas $\Omega_{I I}$ e $\Omega_{I I I}$ é encontrada derivando a equação (6.21) em relação a $h_{i}$, ou seja,

$$
\Omega_{I I}: \quad \sum_{j=1}^{8}(j+1) \cdot\left(h_{i}^{j}-h_{l l_{1}}^{j}\right) \cdot b_{j+1}^{(I I)}=\frac{1}{T_{i}}-\frac{1}{T_{l l_{1}}}
$$

$\mathrm{e}$

$$
\Omega_{I I I}: \quad \sum_{j=1}^{8} j \cdot(j+1) \cdot h_{i}^{j-1} \cdot b_{j+1}^{(I I I)}=-\frac{1}{T_{i}^{2} \cdot c_{P, i}},
$$

onde retomou-se o supraíndice referente a cada sistema. Tem-se agora

$$
b_{j+1}^{(I)}=b_{j+1}^{(I I)}=b_{j+1}^{(I I I)} \quad(j=1,2, \ldots, 8)
$$

Esse vínculo é forte porque impõe à função $s(h, P)$ trazer em sua forma as funções $\left(\frac{\partial s}{\partial h}\right)_{P_{0}}$ e $\left(\frac{\partial^{2} s}{\partial h^{2}}\right)_{P_{0}}$

Os três sistemas juntos têm 14 incógnitas, 8 coeficientes e 6 grandezas termodinâmicas dos espinodais líquido e vapor. Entretanto, devido ao vínculo dado em (6.24), é possível eliminar os coeficientes com a construção de um sistema de equações não-lineares. Em notação matricial os sistemas $\Omega_{I}, \Omega_{I I}$, e $\Omega_{I I I}$ podem ser escritos como

$$
\begin{gathered}
\Omega_{I}: \quad \mathbf{M}^{(I)} \mathbf{b}^{(I)}=\mathbf{y}^{(I)} \\
\Omega_{I I}: \quad \mathbf{M}^{(I I)} \mathbf{b}^{(I I)}=\mathbf{y}^{(I I)} \\
\Omega_{I I I}: \quad \mathbf{M}^{(I I I)} \mathbf{b}^{(I I I)}=\mathbf{y}^{(I I I)}
\end{gathered}
$$


Uma vez que as matrizes $\mathbf{M}^{(I)}, \mathbf{M}^{(I I)}$, e $\mathbf{M}^{(I I I)}$ são invertíveis por construção, os coeficientes são dados por

$$
\begin{gathered}
\mathbf{b}^{(I)}=\left[\mathbf{M}^{(I)}\right]^{-1} \mathbf{y}^{(I)} \\
\mathbf{b}^{(I I)}=\left[\mathbf{M}^{(I I)}\right]^{-1} \mathbf{y}^{(I I)} \\
\mathbf{b}^{(I I I)}=\left[\mathbf{M}^{(I I I)}\right]^{-1} \mathbf{y}^{(I I I)}
\end{gathered}
$$

Dessa forma o vínculo dado por (6.24) é usado para definir a $i$-ésima linha do sistema não-linear $\Omega_{I V}$, conforme Apêndice C, dada por

$$
\Omega_{I V}: \quad f_{i, \min }=\frac{\left[b_{i}^{(I)}+b_{i}^{(I I)}+b_{i}^{(I I I)}\right]^{2}}{b_{i}^{(I)} b_{i}^{(I I)}+b_{i}^{(I)} b_{i}^{(I I I)}+b_{i}^{(I I)} b_{i}^{(I I I)}}-3=0
$$

para $i=1,2, \ldots, 8$. Como

$$
\begin{gathered}
b_{i}^{(I)}=b_{i}^{(I)}\left(h_{l s}, s_{l s}, h_{g s}, s_{g s}\right) \\
b_{i}^{(I I)}=b_{i}^{(I I)}\left(h_{l s}, T_{l s}, h_{g s}, T_{g s}\right) \\
b_{i}^{(I I I)}=b_{i}^{(I I I)}\left(h_{l s}, h_{g s}\right)
\end{gathered}
$$

pode-se escrever

$$
f_{i, \min }\left(h_{l s, s_{l s}}, T_{l s}, h_{g s,} s_{g s}, T_{g s}\right)=0 \quad(i=1,2, \ldots, 8)
$$

Assim, o sistema não-linear $\Omega_{I V}$ possui oito linhas e seis incógnitas. Por ser não-linear o sistema pode apresentar mais de uma solução. Assim além dos vínculos dados por (6.7) e (6.8), vínculos adicionais são usados em busca da solução de (6.10). Cada linha de (6.35) conforme, Apêndice $\mathrm{C}$, tem a propriedade

$$
d f_{i, \min }=0
$$


ou seja,

$$
\left(\frac{\partial f_{i, \min }}{\partial b_{i}^{(I)}}\right)_{b_{i}^{(I I)}, b_{i}^{(I I I)}} d b_{i}^{(I)}+\left(\frac{\partial f_{i, \min }}{\partial b_{i}^{(I I)}}\right)_{b_{i}^{(I)}, b_{i}^{(I I I)}} d b_{i}^{(I I)}+\left(\frac{\partial f_{i, \min }}{\partial b_{i}^{(I I I)}}\right)_{b_{i}^{(I)}, b_{i}^{(I I)}} d b_{i}^{(I I I)}=0
$$

A fim de operacionalizar os cálculos redefiniu-se todas as grandezas termodinâmicas em termos reduzidos, bem como os coeficientes, como mostrado a seguir.

\subsection{Funções reduzidas}

O ponto triplo apresenta a seguinte propriedade

$$
\frac{P_{3} \Delta v_{3}}{R}=T_{3}=\frac{\Delta h_{3}}{\Delta s_{3}}
$$

onde $P_{3}$ é a pressão; $\Delta v_{3}=v_{g 3}-v_{l 3}$, é a diferença entre os volumes específicos do vapor e do líquido; $R$ é a constante dos gases ideais; $T_{3}$ é a temperatura; $\Delta h_{3}=h_{g 3}-h_{l 3}$, é a entalpia específica de vaporização, em que $h_{l 3}=0$ por definição; $\Delta s_{3}=s_{g 3}-s_{l 3}$, é a entropia específica de vaporização, onde $s_{l 3}=0$ por definição [14].

Combinando 247 estados termodinâmicos experimentais obtidos de Kestin [14], entre o ponto triplo e o crítico (inclusives), pode-se construir a Figura 6.17, a qual mostra propriedade de (6.38). Nela cada $i$-ésimo ponto da abscissa da curva em azul é dada por

$$
\frac{P_{i} \Delta v_{i}}{R}=\frac{P_{i}\left(v_{g e, i}-v_{l e, i}\right)}{R}
$$

e na vermelha tem-se

$$
\frac{\Delta h_{i}}{\Delta s_{i}}=\frac{h_{g e, i}-h_{l e, i}}{s_{g e, i}-s_{l e, i}}
$$

para $i=1,2, \ldots, 247$. Note que a propriedade (6.38) tem caráter assintótico, quando o vapor água adquire propriedades de gás ideal. Porém pode ser usada até próximo da pressão ambiente (em destaque na Figura 6.17). 


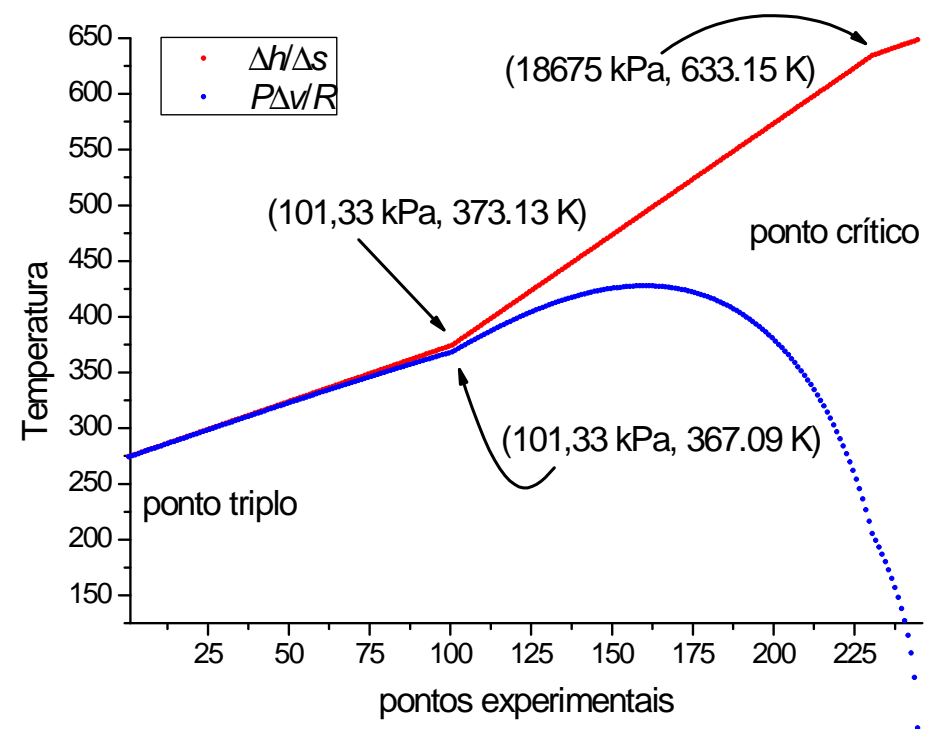

Fig. 6.17- O cálculo de $T=\frac{\Delta h}{\Delta s}$ (em vermelho) e de $T=\frac{P \Delta v}{R}$ (em azul) com pontos experimentais [14], mostra que no ponto triplo $T_{3}=\frac{\Delta h_{3}}{\Delta s_{3}}=\frac{P_{3} \Delta v_{3}}{R}$.

Isto permite definir grandezas termodinâmicas reduzidas ${ }^{12}$ em função do ponto triplo, conforme mostra a Tabela 6.1. Dessa forma cada linha dos sistemas $\Omega_{I}, \Omega_{I I}, \Omega_{I I I}$ (equações (6.21) (6.22) e (6.23), respectivamente) é reescrita como

$$
\begin{gathered}
\Omega_{I}: \quad \sum_{j=1}^{8} c_{j}^{(I)} \cdot\left[h_{r, i}^{j+1}-j h_{r, i} h_{r l l_{1}}^{j}+j h_{r l l_{1}}^{j+1}\right]=\left(s_{r, i}-s_{r l l_{1}}\right)-\frac{\left(h_{r, i}-h_{r l l_{1}}\right)}{T_{r l l_{1}}}, \\
\Omega_{I I}: \quad \sum_{j=1}^{8} c_{j}^{(I I)} \cdot(j+1) \cdot\left(h_{r, i}^{j}-h_{r l l_{1}}^{j}\right)=\frac{1}{T_{r, i}}-\frac{1}{T_{r l l_{1}}}
\end{gathered}
$$

e

$$
\Omega_{I I}: \quad \sum_{j=1}^{8} c_{j}^{(I I I)} \cdot j \cdot(j+1) \cdot h_{r, i}^{j-1}=-\frac{1}{T_{r, i}^{2} \cdot c_{r, i}},
$$

onde $c_{j}^{(k)}$ são os coeficientes dados por

$$
c_{j}^{(k)}=b_{j+1}^{(k)} \frac{h_{3}^{j}}{s_{3}}(j=1,2, \ldots, 8 \text { e } k=I, I I, I I I)
$$

12 O calor específico reduzido $c_{p r}$ ou simplesmente $c_{r}$ é definido em função da entropia pois $c_{p}=\left(\frac{\partial h}{\partial T}\right)_{P}=$ $\frac{h_{3}}{T_{3}}\left(\frac{\partial h_{r}}{\partial T_{r}}\right)_{P}=s_{3}\left(\frac{\partial h_{r}}{\partial T_{r}}\right)_{P}$ e daí $c_{r}=\frac{c_{p}}{s_{3}}$. 


\begin{tabular}{|c|l|}
\hline$s_{r}=\frac{s}{s_{3}}$ & entropia específica reduzida \\
\hline$h_{r}=\frac{h}{h_{3}}$ & entalpia específica reduzida \\
\hline$T_{r}=\frac{T}{T_{3}}$ & temperatura específica reduzida \\
\hline$c_{r}=\frac{c_{p}}{s_{3}}$ & calor específico reduzido \\
\hline$v_{r}=v \frac{R}{s_{3} \Delta v_{3}}$ & volume específico reduzido \\
\hline
\end{tabular}

Tabela 6.1- Grandezas reduzidas

\subsection{Algoritmo}

\subsubsection{Sobre a obtenção dos dados experimentais}

Conhece-se a pressão $P_{0}$ do estado metaestável em que o sistema se encontra. A partir daí, com ajuda do algoritmo da IAPWS [34][35], obtém-se todas as grandezas termodinâmicas necessárias para a construção dos sistemas $\Omega_{I}, \Omega_{I I}, \Omega_{I I I}$.

1. Impõe-se que esta é a pressão de vapor, ou seja, $P_{0} \equiv P_{e}$.

2. Calcula-se a temperatura no equilíbrio de fases, $T_{e}=T\left(P_{e}\right)$.

3. Calcula-se as seguintes grandezas, todas em função de $\left(P_{e}, T_{e}\right)$, ou seja, $h_{l e}, s_{l e}, c_{p, l e}, g_{l e}$ e $v_{l e}$ para o líquido e $h_{g e}, s_{g e}, c_{p, g e}, g_{g e}$ e $v_{g e}$ para o vapor no equilíbrio de fases.

4. Calcula-se a entalpia do líquido no estado metaestável, $h_{l m}=h_{l e}+0.05\left(h_{g e}-h_{l e}\right)$ (limite dado pela IAPWS) e em seguida a temperatura $T_{l m}=T\left(h_{l m}, P_{e}\right)$.

5. Calcula-se as seguintes grandezas em função de $\left(P_{e}, T_{l m}\right), s_{l m}, c_{p, l m}, g_{l m}$ e $v_{l m}$ para o líquido metaestável..

6. Calcula-se em função de $\left(P_{e}, T_{l m}\right)$ as seguintes grandezas para o vapor estável, $h_{g g}, s_{g g}$, $c_{p, g g}, g_{g g}$ e $v_{g g}$ 
7. Calcula-se a entalpia do vapor no estado metaestável, $h_{g m}=h_{l e}+0.95\left(h_{g e}-h_{l e}\right)$ (limite dado pela IAPWS) e em seguida a temperatura $T_{g m}=T\left(h_{g m}, P_{e}\right)$.

8. Calcula-se as seguintes grandezas em função de $\left(P_{e}, T_{g m}\right), s_{g m}, c_{p, g m}, g_{g m}$ e $v_{g m}$ para o líquido metaestável..

9. Calcula-se em função de $\left(P_{e}, T_{l m}\right)$ as seguintes gradezas para o líquido estável, $h_{l l}, s_{l l}$, $c_{p, l l}, g_{l l}$ e $v_{l l}$

10. Calcula-se o ponto do líquido estável de referência $h_{l l 1}=2 h_{l l}-h_{l e}$ e em seguida a temperatura, $T_{l l 1}=T\left(h_{l l 1}, P_{e}\right)$

11. Para o estado $l l_{1}$ calcula-se as seguintes grandezas em função de $\left(P_{e}, T_{l l 1}\right), s_{l l 1}, c_{p, l l 1}, g_{l l 1} \mathrm{e}$ $v_{l l 1}$.

A Tabela 6.2 abaixo relaciona os pontos semi-experimentais utilizados no algoritmo. Note que é conhecido a priori o calor específico do líquido e do vapor no espinodal para qualquer pressão entre o ponto triplo e o crítico. A simetria nos pontos é intencional.

\begin{tabular}{|c|c|c|c|c|c|}
\hline Ponto & subíndice & $h$ & $s$ & $T$ & $c_{P}$ \\
\hline 0 & $l l_{1}$ & $2 h_{l l}-h_{l e}$ & $s\left(T_{l l 1}\right)$ & $T\left(h_{l l 1}\right)$ & $c_{P}\left(T_{l l 1}\right)$ \\
\hline 1 & $l l$ & $h\left(T_{g m}\right)$ & $s\left(T_{g m}\right)$ & $T_{g m}$ & $c_{P}\left(T_{g m}\right)$ \\
\hline 2 & $l e$ & $h_{l e}$ & $s_{l e}$ & $T\left(P_{0}\right)$ & $c_{P, l e}$ \\
\hline 3 & $l m$ & $h_{l e}+0.05 h_{v a p}$ & $s\left(T_{l m}\right)$ & $T\left(h_{l m}\right)$ & $c_{P}\left(T_{l m}\right)$ \\
\hline 4 & $l s$ & $h_{l s}$ & $s_{l s}$ & $T_{l s}$ & $+\infty$ \\
\hline 5 & $g s$ & $h_{g s}$ & $s_{g s}$ & $T_{g s}$ & $+\infty$ \\
\hline 6 & $g m$ & $h_{l e}+0.95 h_{v a p}$ & $s\left(T_{g m}\right)$ & $T\left(h_{g m}\right)$ & $c_{P}\left(T_{g m}\right)$ \\
\hline 7 & $g e$ & $h_{g e}$ & $s_{g e}$ & $T\left(P_{0}\right)$ & $c_{P, g e}$ \\
\hline 8 & $g g$ & $h\left(T_{l m}\right)$ & $s\left(T_{l m}\right)$ & $T_{l m}$ & $c_{P}\left(T_{l m}\right)$ \\
\hline
\end{tabular}

Tabela 6.2- Pontos utilizados na construção dos sistemas $\Omega_{I}, \Omega_{I I}$, e $\Omega_{I I I}$.

Em alusão ao diagrama $P, v$, é esperado que os espinodais estejam dentro da região de coexistência, entre os binodais. Dessa forma, as grandezas nos espinodais foram fornecidas nos 
intervalos dados pela Tabela 6.3. O algoritmo busca valores dos espinodais em que se verifique a eq.(6.35) para cada coeficiente com uma tolerância definida previamente.

\begin{tabular}{|c|c|c|c|}
\cline { 2 - 4 } \multicolumn{1}{c|}{} & $h(\mathrm{~kJ} / \mathrm{kg})$ & $s(\mathrm{~kJ} / \mathrm{kg} / \mathrm{K})$ & $T(\mathrm{~K})$ \\
\hline$l s$ & $h_{l e}<h_{l s}<h_{c}$ & $s_{l e}<s_{l s}<s_{c}$ & $T_{e}<T_{l s}<T_{c}$ \\
\hline$g s$ & $h_{c}<h_{g s}<h_{g e}$ & $s_{c}<s_{g s}<s_{g e}$ & $T_{g s}<T_{c}$ \\
\hline
\end{tabular}

Tabela 6.3- Pontos espinodais obtidos

Evidentemente que dependendo do passo (de cada grandeza) e da tolerância (sobre os coeficientes) é encontrada mais de uma solução. É necessário, portanto, a verificação da solução (grandezas espinodais e coeficientes) que induz menor erro de interpolação.

\subsection{Resultados Preliminares}

Fornecendo o valor da pressão $P_{e}=87.69 \mathrm{kPa}$ o algoritmo da IAPWS fornece os dados para a interpolação reunidos na Tabela 6.4, dada a seguir.

\begin{tabular}{|c|c|c|c|c|c|}
\cline { 2 - 6 } \multicolumn{1}{c|}{} & $T(\mathrm{~K})$ & $h(\mathrm{~kJ} / \mathrm{kg})$ & $s(\mathrm{~kJ} / \mathrm{kg} / \mathrm{K})$ & $c_{p}(\mathrm{~kJ} / \mathrm{kg} / \mathrm{K})$ & $g(\mathrm{~kJ} / \mathrm{kg})$ \\
\hline$l l_{1}$ & 313.25 & 168.00 & 0.57369 & 4.1786 & -11.707 \\
\hline$l l$ & 320.93 & 200.11 & 0.67497 & 4.1792 & -16.507 \\
\hline$l e$ & 363.12 & 376.85 & 1.1923 & 4.2050 & -56.113 \\
\hline$l m$ & 379.37 & 445.33 & 1.3768 & 4.2251 & -76.995 \\
\hline$g m$ & 313.25 & 2545.4 & 7.1390 & 2.8215 & 309.07 \\
\hline$g e$ & 363.12 & 2659.5 & 7.4784 & 2.0415 & -56.101 \\
\hline$g g$ & 417.71 & 2768.1 & 7.7572 & 1.9649 & -472.19 \\
\hline
\end{tabular}

Tabela 6.4- Pontos utilizados na construção dos sistemas

Os dados referentes à energia livre de Gibbs específica são usados pelo algoritmo a fim de minimizar erros de interpolação.

Com as grandezas termodinâmicas reduzidas, hrls, srls, Trls, hrgs, srgs, e Trgs, variando entre os limites dados acima, a um passo da ordem de $10^{-3}$, e os coeficientes $c_{0}, c_{1}, \ldots, c_{9}$, dados pela eq. (6.44), aceitos com tolerância de até $20 \%$ de desvio (definido sobre a eq. (6.24)), o algoritmo fornece os espinodais e os coeficientes conforme as Tabelas 6.5 e 6.6. Os respec- 
tivos desvios foram calculados sobre todas as soluções que resultaram menores resíduos sobre os quatro sistemas.

\begin{tabular}{|c|c|c|c|}
\cline { 2 - 4 } \multicolumn{1}{c|}{} & $h_{r}(\mathrm{~kJ} / \mathrm{kg})$ & $s_{r}(\mathrm{~kJ} / \mathrm{kg} / \mathrm{K})$ & $T_{r} \mathrm{~s}(\mathrm{~K})$ \\
\hline$l s$ & $0.47 \pm 0.01$ & $0.320 \pm 0.005$ & $2.08 \pm 0.04$ \\
\hline$g s$ & $0.894 \pm 0.002$ & $0.646 \pm 0.002$ & $0.968 \pm 0.007$ \\
\hline
\end{tabular}

Tabela 6.5- Pontos espinodais obtidos

\begin{tabular}{|c|c|c|}
\hline$c_{j}$ & $\left(=b_{j} \frac{h_{3}^{j}}{s_{3}}\right)$ & $\epsilon_{j}$ \\
\hline$c_{0}$ & 0.00042 & 0 \\
\hline$c_{1}$ & 0.97929 & 0.00004 \\
\hline$c_{2}$ & -0.6987 & 0.0008 \\
\hline$c_{3}$ & -2.29 & 0.01 \\
\hline$c_{4}$ & 18.78 & 0.07 \\
\hline$c_{5}$ & -62.0 & 0.3 \\
\hline$c_{6}$ & 107.2 & 0.7 \\
\hline$c_{7}$ & -97 & 1 \\
\hline$c_{8}$ & 43.3 & 0.6 \\
\hline$c_{9}$ & -7.4 & 0.2 \\
\hline
\end{tabular}

Tabela 6.6- Coeficientes do polinômio interpolador.

Com os coeficientes calculados, tem-se a eq. (6.10). A Figura 6.18 representa a função entropia reduzida $s_{r}\left(h_{r}, P_{0}\right)$ para a pressão $P_{0}=87.69 \mathrm{kPa}$, com os coeficientes dados pela Tabela 6.6, calculada no intervalo $\left(h_{r l e}, h_{r g e}\right)$. A linha em azul é a linha de saturação (isoterma).

Na Figura 6.19 tem-se o gráfico da temperatura reduzida no mesmo intervalo, calculada como $T_{r}=1 /\left(\partial s_{r} / \partial h_{r}\right)_{P_{0}}$. Usando a eq. (6.7) para integrar o inverso da temperatura sobre o intervalo $\left(h_{r l e}, h_{r g e}\right)$, resulta área igual a $3.341054 \times 10^{-5}$; dada a tolerância sobre o cálculo dos coeficiente, tem-se a verificação da construção de Maxwell com boa aproximação.

E o limite de estabilidade da matéria, caracterizado pela divergência do calor específico a pressão constante, é verificado na Figura 6.20. O calor específico a pressão constante reduzido 


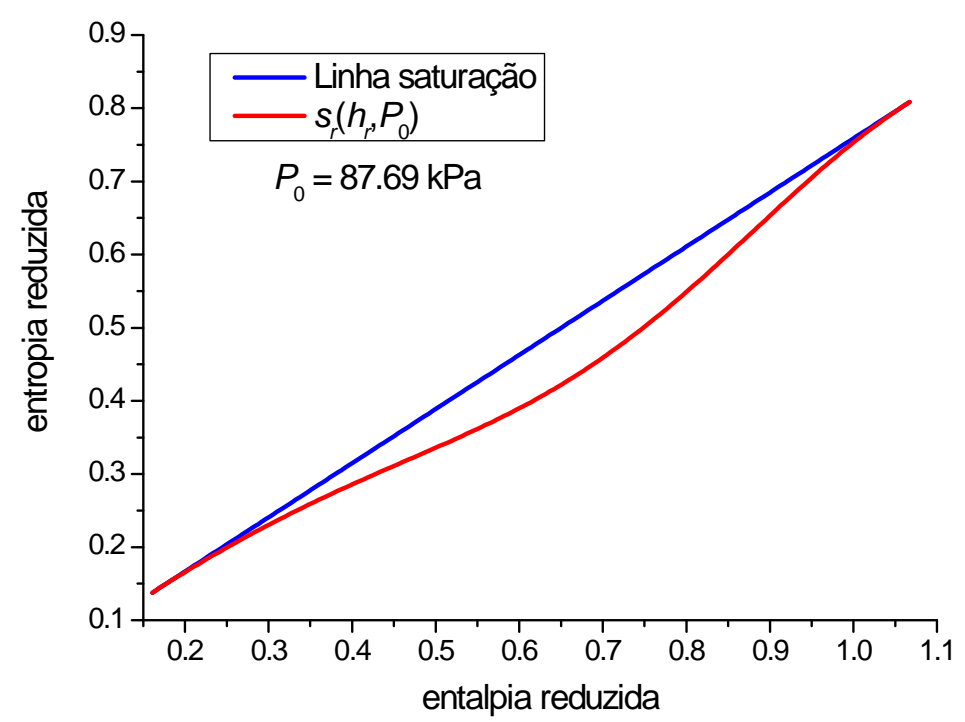

Fig. 6.18- Em vermelho tem-se a curva isobárica $s\left(h, P_{0}\right)$ e em azul a curva isobárica e isotérmica que caracteriza a região metaestável do fluido.

foi calculado combinando as eqs. (6.1) e (6.2) como

$$
c_{p r}=-\frac{\left[\left(\frac{\partial s_{r}}{\partial h_{r}}\right)_{P_{0}}\right]^{2}}{\left(\frac{\partial^{2} s_{r}}{\partial h_{r}^{2}}\right)_{P_{0}}}
$$

e calculado no intervalo $\left(h_{r l e}, h_{r g e}\right)$. Na Figura 6.20 a linha azul é uma linha guia mostrando que para no binodal do líquido $c_{p r}\left(h_{r l e}, P_{0}\right)=c_{p r l e}$; a linha verde também é guia, indicando que no binodal do vapor $c_{p r}\left(h_{r g e}, P_{0}\right)=c_{p r g e}$. Vale observar que entre os espinodais o calor específico assume valores negativos, como era esperado.

A Figura 6.21 mostra algumas isóbaras obtidas através de cálculos preliminares, calculadas entre os respectivos binodais $l e\left(P_{e}, T_{e}\right)$ e $g e\left(P_{e}, T_{e}\right)$. Nota-se que com o aumento da pressão, a curvatura de cada isóbara da função entropia, $s\left(h, P_{0}\right)$, torna-se menos côncava e os binodais aproximam-se mais, como era esperado.

A eq. (6.10) usada na definição da energia de Gibbs evidencia o fim da barreira energética como mostra a Figura 6.22. Nela são mostradas três curvas isóbaras, em que se evidencia o fim da barreira para a temperatura no espinodal do vapor, $T_{g s}$. 


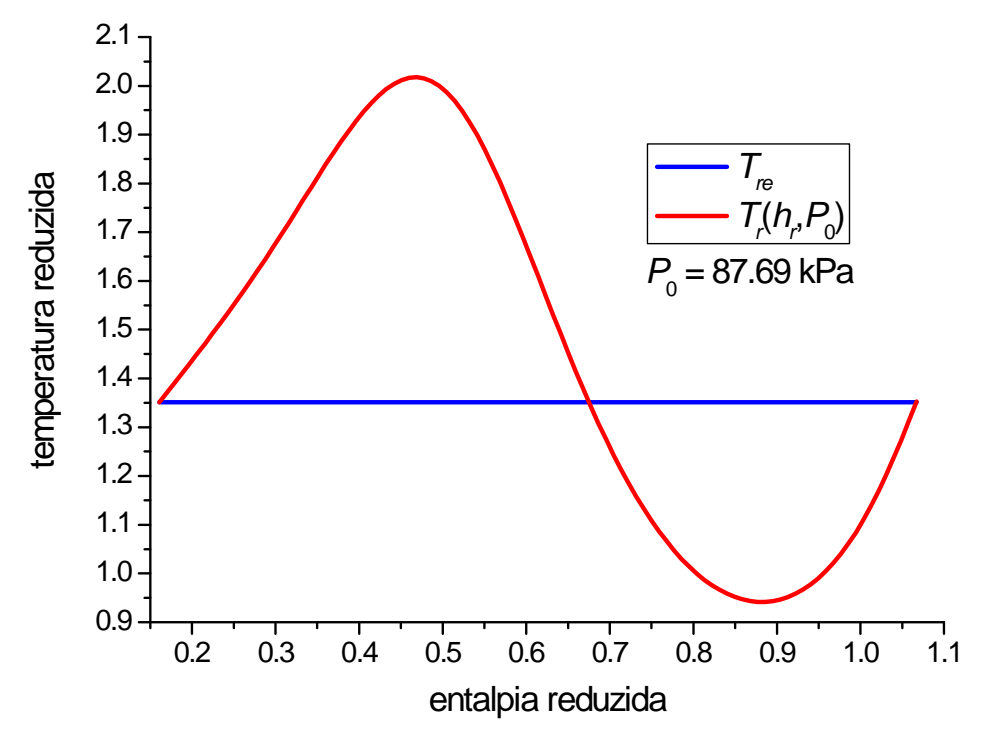

Fig. 6.19- Em azul tem-se a temperatura de transição de fase, $T_{r e}$.

\subsubsection{Nucleação}

Os experimentos de Viisanen e Strey foram executados em torno do ponto triplo, como mostrado a seguir

$$
\begin{gathered}
150<T(/ \mathrm{K})<310 \\
70<P(/ \mathrm{Pa})<3500
\end{gathered}
$$

Por razões ainda não determinadas o algoritmo falhou nesta região. Entretanto, obteve-se a função entropia, com considerável desvio, para a pressão $P_{0}=3.778 \mathrm{kPa}$, conforme o algoritmo acima. Foi aplicada ao cálculo do núcleo crítico, eq. (5.32), e comparada com o algoritmo de Dillmann-Meier [4][5] (vide Figura 6.23).

Novamente o comportamento da eq. (5.32), em que a constante de proporcionalidade foi assumida unitária, $\phi=1$, e a capacidade térmica molar a volume constante foi considerada constante na região metaestável, $C_{v}=3 R / 2$, é qualitativamente similar ao experimental. Como mostrado nas Figuras 5.13 e 6.23, a comparação da eq. (5.32) com dados experimentais dá-se mediante um fator da ordem de $10^{3}$. Portanto, é forte o indício de que $\phi>1$, como previsto na dissertação de mestrado de Pasqua [23]. 


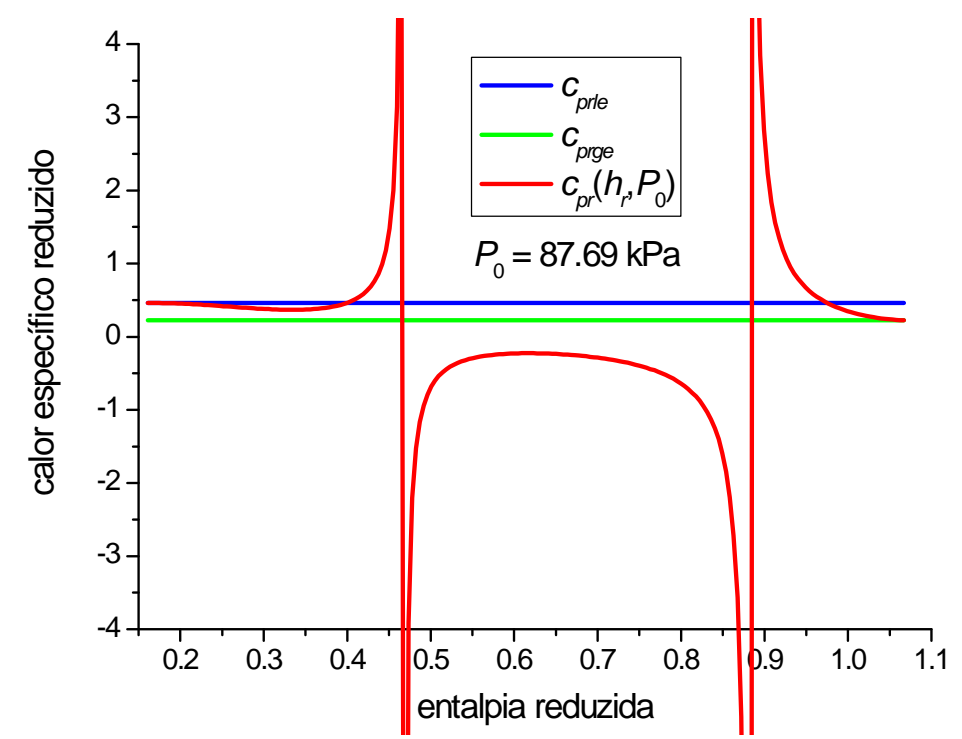

Fig. 6.20- Em azul tem-se o crle, e em verde crge.

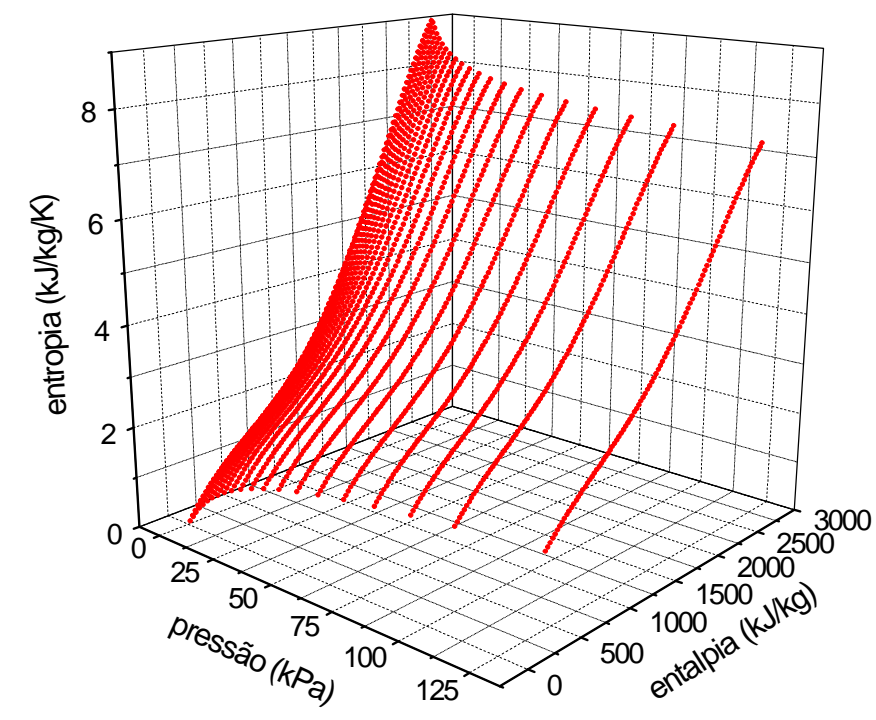

Fig. 6.21- À medida que a pressão aumenta, verifica-se a aproximação do binodais e a diminuição da concavidade da curva, como era esperado. 


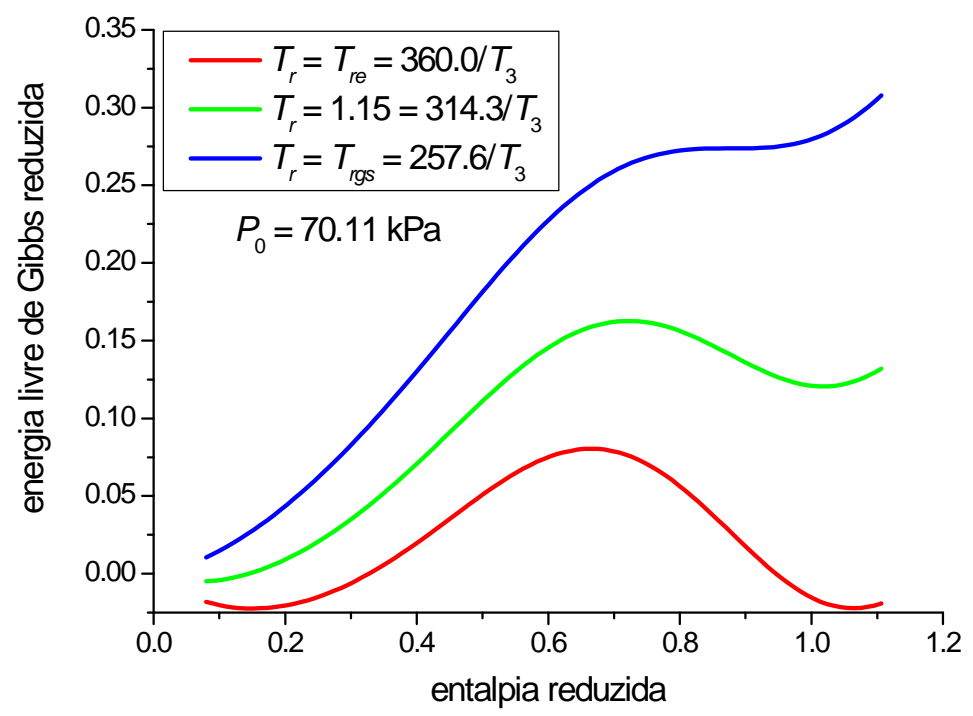

Fig. 6.22- Fim da barreira em $T_{g s}=257.6 \mathrm{~K}$ para a pressão $P_{0}=70.11 \mathrm{kPa}$.

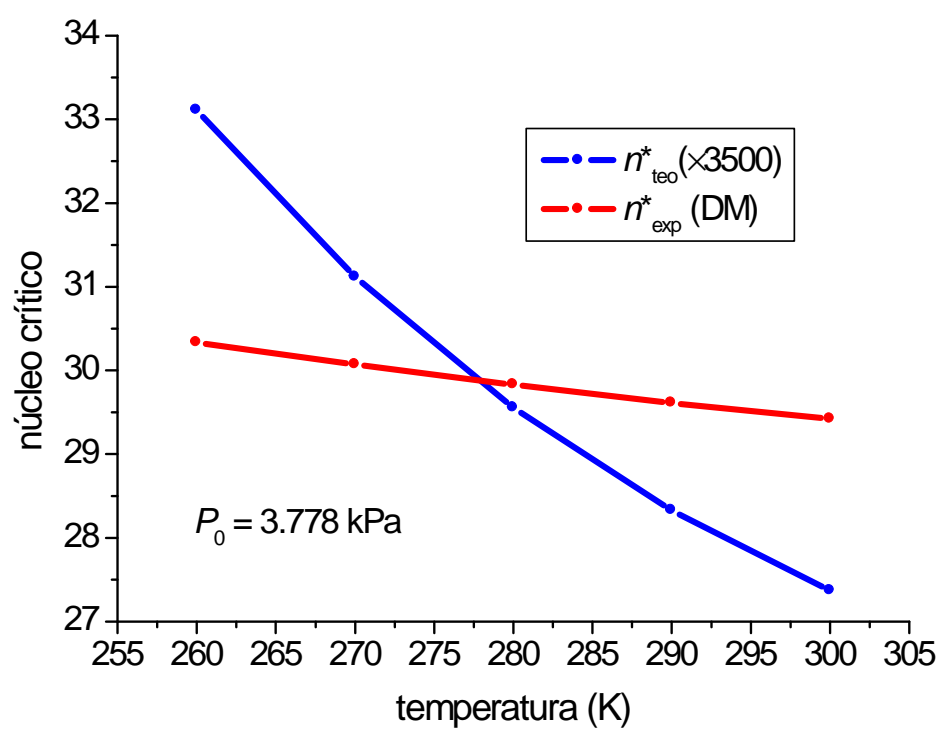

Fig. 6.23- O cálculo de $n^{*}$ via função entropia $s\left(h, P_{0}\right)$ considerou $\phi=1$ e $C_{v}=3 R / 2$, na eq. (5.26). 


\section{7 \\ Tamanho do Núcleo Crítico (Einstein)}

Para lidar com aglomerados e núcleos em uma abordagem termodinâmica, um ensemble a pressão constante é o mais apropriado, uma vez que experimentos são usualmente realizados sob condições de pressão constante. A variável conjugada da pressão é o volume, em que se verifica que a função de partição deste ensemble requer a soma discreta sobre volumes não especificados. Como essa soma pode levar a erros, principalmente em estados próximos do espinodal, tem-se a necessidade de uma correta escala de volume principalmente quando se lida com sistemas pequenos. Este problema foi resolvido por Koper e Reiss [15] para flutuações isotérmicas em um fluido ideal. Esta teoria pôde ser estendida para flutuações não isotérmicas, como por exemplo, flutuações em um fluido não-ideal metaestável mantido a pressão constante $P_{0}[24][22]$.

Seja um sistema mantido à pressão $P_{0}$, que se encontra em um estado metaestável $\mathrm{gm}$, com temperatura $T_{g m}=T_{0}$. Um subsistema (elemento de volume) consistindo de $n$ monômeros, ocupando um volume médio $\bar{V}_{g m}$, com entalpia $h_{g m}$, entropia $s_{g m}$, terá uma flutuação média $\langle\Delta V\rangle_{g}$ entre $\bar{V}_{g m}$ e $V$, onde $V \geq V_{g s}$, dada por

$$
\langle\Delta V\rangle_{g}=\int_{V_{g m}}^{V} V^{\prime} \Pi_{g}\left(V^{\prime}, P_{0}, T_{0}\right) d V^{\prime}
$$

onde $\Pi_{g}$ é a probabilidade de flutuação da fase gasosa dada por

$$
\Pi_{g}\left(V^{\prime}, P_{0}, T_{0}\right)=\frac{\widetilde{P}\left(n, V^{\prime}, T_{0}\right)}{k_{B} T_{0}} \exp \left\{-\frac{\Delta H_{g}-T_{0} \Delta S_{g}}{k_{B} T_{0}}\right\}
$$

onde $\widetilde{P}$ é um fator relacionado à pressão interna do elemento de volume e onde

$$
\Delta H_{g}=H\left(n, V^{\prime}, T_{60}\right)-H\left(n, \bar{V}_{g m}, T_{0}\right)
$$


e

$$
\Delta S_{g}=S\left(n, V^{\prime}, T_{0}\right)-S\left(n, \bar{V}_{g m}, T_{0}\right)
$$

Se a magnitude da flutuação é maior ou igual à diferença de volume entre $\bar{V}_{g m}$ e $V_{g s}$,

$$
\langle\Delta V\rangle_{g} \geq\left(\bar{V}_{g m}-V_{g s}\right)=n\left(\bar{v}_{g m}-v_{g s}\right)
$$

onde $v$ é o volume por partícula, então tem-se que esta quantidade de vapor tornar-se-á totalmente instável e mudará de fase. E o aglomerado da nova fase encontrar-se-á no estado $l l$, com volume médio $\bar{V}_{l l}$, entalpia $h_{l l}$, entropia $s_{l l}$, e temperatura $T_{l l}=T_{0}$.

O aglomerado da nova fase ocupando um volume médio $\bar{V}_{l l}$ terá uma flutuação média $\langle\Delta V\rangle_{l}$ dada por

$$
\langle\Delta V\rangle_{l}=\int_{V_{l l}}^{V} V^{\prime} \Pi_{l}\left(V^{\prime}, P_{0}, T_{0}\right) d V^{\prime}
$$

onde $\Pi_{l}$ é a probabilidade de flutuação da fase líquida dada por

$$
\begin{gathered}
\Pi_{l}\left(V^{\prime}, P_{0}, T_{0}\right)=\frac{\widetilde{P}\left(n, V^{\prime}, T_{0}\right)}{k_{B} T_{0}} \exp \left\{-\frac{\Delta H_{l}-T_{0} \Delta S_{l}}{k_{B} T_{0}}\right\} \\
\Delta H_{l}=H\left(n, V^{\prime}, T_{0}\right)-H\left(n, \bar{V}_{l l}, T_{0}\right) \\
\Delta S_{l}=S\left(n, V^{\prime}, T_{0}\right)-S\left(n, \bar{V}_{l l}, T_{0}\right)
\end{gathered}
$$

O aglomerado agora na fase líquida, não decairá, somente se a flutuação média for menor que a diferença de volume entre $\bar{V}_{l l}$ e $V_{l s}$, ou seja,

$$
\langle\Delta V\rangle_{l} \leq\left(\bar{V}_{l l}-V_{l s}\right)=n\left(\bar{v}_{l l}-v_{l s}\right)
$$

Se $n$ é o número de monômeros que forma o núcleo crítico, então deve satisfazer as desigualdades acima simultaneamente, o que permite escrever

$$
\frac{\langle\Delta V\rangle_{g}}{\left(\bar{v}_{g m}-v_{g s}\right)} \geq n^{*} \geq \frac{\langle\Delta V\rangle_{l}}{\left(\bar{v}_{l l}-v_{l s}\right)}
$$

expressão esta que por iteração fornece o tamanho do núcleo crítico. 
Entretanto, o algoritmo para o cálculo do núcleo crítico, $n^{*}$, ainda não foi desenvolvido pois a função entropia dada por (6.10) ainda não fornece de forma segura o volume na região metaestável. Assim, a verificação da eq. (7.11) ficou momentaneamente postergada. 


\section{8 \\ Conclusões e Perspectivas}

A existência do estado metaestável é devida a vínculos internos do sistema, criado por processos termodinâmicos suficientemente rápidos. O fenômeno da nucleação inicia-se quando o vínculo interno, entendido como uma barreira energética $\left(\Delta G^{*}\right)$, é rompido devido a flutuações térmicas de determinada magnitude que desestabilizam um elemento de volume e por conseqüência todo o sistema.

Uma das características fundamentais da transição de fase de primeira ordem é a exigência de calor latente para sua complementação, implicando na variação de entropia, devido ao rearranjo estrutural do fluido. Se o sistema é mantido a pressão constante e a temperatura final é igual à inicial, o processo é suficientemente bem analisado com as grandezas termodinâmicas entalpia e a entropia. Portanto, é natural ter a entalpia e a pressão como variáveis da entropia: $S(H, P)$. O diagrama de Mollier modificado para evidenciar a região metaestável ajudou a desenvolver uma abordagem teórica para estudar o fenômeno da nucleação, a Teoria Entrópica da Nucleação. Mediante uma teoria da flutuação (Landau), no seu aspecto mais simplificado, foi determinado o tamanho do núcleo crítico, eq. (5.26). Tal expressão exige conhecimento da entalpia, da temperatura e do calor específico a volume constante do estado metaestável e do espinodal do líquido e do vapor, e também a determinação da constante $\phi$. Com alguma adequação a expressão para o núcleo crítico, eq. (5.26), é aplicada não só para a nucleação, mas também para a denucleação (vaporização).

Para a análise da TEN escolheu-se o fluido puro água. É um fluido complexo de importância inquestionável, muito estudado, apresentando vasta quantidade de dados experimentais de estado estáveis e principalmente de coexistência de fases. Porém muito pouco sobre estados 
metaestáveis das fases líquida (em relação ao vapor) e gasosa (em relação ao líquido). Com relação a esta o artigo da IAPWS chega a declarar que não há dados experimentais disponíveis $[34][35]$.

Inicialmente, adotou-se o vapor de água como um gás ideal para a obtenção do calor específico a volume constante. Usou-se as equações de estado de van der Waals para obtenção do volume e da temperatura no espinodal e, funções residuais para o cálculo na entalpia nesse ponto [23]. Os resultados comparados com a experiência encontrada na literatura [33][36] mostraram que o tamanho do núcleo crítico era em média três ordens de grandeza menor que o experimental [23]. Em seguida, adotou-se a equação de estado de Peng-Robinson, reconhecida pela sua acurácia e amplamente usada. O resultado foi o tamanho do núcleo crítico também em média três ordens de grandeza menor que o experimental. Porém ambas equações de estado mostraram-se inadequadas nessa representação (processo isobárico). São equações dedicadas a estados de um fluido na fase gasosa, mostrando pouca acurácia para a fase líquida. A região metaestável do fluido é topologicamente correta, verifica-se a construção de Maxwell, pressões negativas para a fase líquida no estado metaestável. Porém, o loop de van der Waals não é suficientemente bem dimensionado. Os espinodais encontram-se deslocados, resultando temperaturas questionáveis nesses pontos. Cabe notar que o loop de van der Waals na fase líquida é mais profundo na EE-PR, quando comparado com a EE-vdW, o que significa que para uma mesma temperatura, a pressão no espinodal daquela é menor do que a desta. A deficiência da EE-vdW e da EE-PR para a região metaestável deve-se ao restrito número de constantes. Mesmo considerando o sofisticado algoritmo do IAPWS, os estados da região metaestável da água são suficientemente bem representados apenas em torno dos binodais [34][35].

Para estudar o fenômeno da nucleação tornou-se desejável uma função de estado entropia, cujas variáveis canônicas são a entalpia e a pressão, $S(H, P)$, a qual permita analisar o sistema na região metaestável de um processo isobárico. Deve trazer em sua estrutura $(i)$ a continuidade 
de estados do fluido, em que se verifique a construção de Maxwell, desde o ponto triplo ao ponto crítico; (ii) o limite de estabilidade termodinâmica, caracterizado pela divergência do calor específico a pressão constante no limite do espinodal (geometricamente é um ponto de inflexão da função entropia); (iii) a temperatura em cada ponto da região metaestável até o espinodal.

A solução encontrada foi obter a função entropia por interpolação em que aspectos físicos e geométricos foram determinantes. Um sistema não-linear homogêneo foi obtido a partir de três sistemas de equações lineares não-homogêneos. Os resultados preliminares são satisfatórios: ( $i$ ) as propriedades acima mencionadas são observadas nesta função polinomial, ou seja, a continuidade de estados gasoso e líquido, o limite de estabilidade, a construção de Maxwell; (ii) o calor específico a pressão constante como função da entalpia e apresenta divergência no espinodal.

Os novos cálculos do núcleo crítico com a função entropia mostraram concordância qualitativa em relação à dependência da temperatura. Porém há discrepância em relação a ordem de grandeza, indicando que a função do núcleo crítico está incompleta. É forte o indício de que $\phi>1$, conforme previsto na dissertação de mestrado de Pasqua [23]. A teoria da flutuação de Landau diz respeito a pequenas flutuações em torno do ponto médio. Entende-se que estes resultados preliminares, obtidos através da teoria da flutuação de termodinâmica de equilíbrio (Landau), quando aplicada a sistemas pequenos não pode dar resultados seguros, uma vez que para sistemas pequenos, parâmetros de superfície alteram os resultados quando comparados com sistemas macroscópicos. Além do mais, sistemas pequenos estão longe do limite termodinâmico (inferior) e parâmetros intrínsecos não estão bem definidos. Ainda assim, seria interessante rever a eq. (5.26) a partir de uma adequada expressão para a flutação entalpia, e calcular a constante $\phi$. 
Para lidar com aglomerados e núcleos em uma abordagem termodinâmica, um ensemble a pressão constante é o mais apropriado, uma vez que experimentos são usualmente realizados sob condições de pressão constante. A variável conjugada da pressão é o volume e, neste ensemble é exigido a soma discreta sobre volumes discretos não especificados. Como tal soma pode levar a erros consideráveis, tem-se a necessidade de uma correta escala de volume principalmente quando se lida com sistemas pequenos. Este problema foi resolvido por Koper e Reiss para flutuações isotérmicas em um fluido ideal. Esta teoria pôde ser estendida para flutuações não isotérmicas, como por exemplo, flutuações em um fluido não-ideal metaestável mantido a pressão constante $P_{0}$ e temperatura constante $T_{0}$. Os parâmetros termodinâmicos da quantidade de matéria que está submetida a uma flutuação mudam e diferem daquela do banho (macroscópico), acordando a equação de estado $S=S\left(H, P_{0}\right)$ (mesoscópico). Estas flutuações não são necessariamente isotérmicas e não necessitam ser tratadas como em um fluido ideal.

Essa nova abordagem para tratar do fenômeno da nucleação, baseada na flutuação de Einstein, reforça a importância da função entropia e exige mais dela. É necessário obter a superfície $S(H, P)$, visto que o volume é encontrado com a variação da entalpia em relação à pressão, com a entropia mantida constante, $V=(\partial H / \partial P)_{S}$.

Obtendo o valor do núcleo crítico, $n^{*}$, via TEN, com auxílio da função entropia, objetivase

- encontrar a altura da barreira de energia, $\Delta G^{*}$, em que novamente a função entropia poderá auxiliar, visto que $\Delta G=\Delta H-T \Delta S$, em que $T=(\partial H / \partial S)_{P_{0}}$ e $S=S\left(H, P_{0}\right)$ e verificar $\Delta G^{*} \propto n^{*}$

- encontrar a taxa de nucleação $J=J_{0} \exp \left[-\Delta G^{*} /\left(k_{B} T\right)\right]$ e verificar $\left(\partial \ln J / \partial \ln S_{s}\right)_{T} \simeq$ $n^{*}$, relação esta que segundo Viisanen e Strey, é independente do modelo. 
Apêndices 


\section{A \\ Da incompleteza da função $F(P, v, T)$}

Propõe-se aqui evidenciar a incompleteza das equações de estado do tipo van der Waals. Ainda que topologicamente corretas, a região metaestável apresenta desvios significativos e, como será mostrado, a entropia não é completamente determinada por funções $F(P, v, T)=0$, pois está relacionada com o calor específico, o qual é dado experimental do fluido.

\section{A.1 Equação de Estado Fundamental}

Considerando um sistema em que não se verifica variação na energia cinética e na energia potencial gravitacional, e não há outros campos, então, a variação da energia do sistema deve-se a processos internos que envolvem mudança de temperatura, de pressão e de volume, acompanhados ou não de mudança de fase e de reação química [14]. Dessa forma, a Primeira Lei da Termodinâmica combinada com a primeira parte da Segunda Lei da Termodinâmica, equação (2.16), resulta uma função que depende da entropia e do volume, $U=U(S, V)$. Em termos específicos, a variação da energia interna específica resulta

$$
d u=T d s-P d v
$$

onde $u=U / m, s=S / m, v=V / m$, ou

$$
d u=\left(\frac{\partial u}{\partial s}\right)_{v} d s+\left(\frac{\partial u}{\partial v}\right)_{s} d v
$$

o que permite identificar

$$
\left(\frac{\partial u}{\partial s}\right)_{v}=T(s, v) \text { e }\left(\frac{\partial u}{\partial v}\right)_{s}=-P(s, v)
$$

As equações (A.3) constituem relações que devem necessariamente ser satisfeitas pelas propriedades de um sistema simples em qualquer estado; do contrário estariam inconsistentes 
com a Segunda Lei da Termodinâmica e deveria, portanto, ser possível projetar uma máquina de movimento perpétuo de segunda espécie, retirando trabalho do fluido (substância) [14].

Supondo haver uma função $u=u(s, v)$, obtida por interpolação de um conjunto de dados experimentais, deveria ser possível obter todas as propriedades usadas em análise de estados de um simples sistema, além de $P$ e $T$, ou seja, entalpia, $h$, calor específico a pressão constante, $c_{p}$, e calor específico a volume constante, $c_{v}$. Além disso, obtendo $P$ e $T$ por simples diferenciação de $u(s, v)$, deveria ser possível, a partir delas, eliminar a entropia e obter

$$
F(P, v, T)=0
$$

ou seja, equação de estado, onde $T$ é a temperatura termodinâmica (absoluta). Portanto, $u=$ $u(s, v)$ constitui uma equação de estado fundamental, porém de utilidade limitada devido a dificuldades experimentais ${ }^{13}$.

Agora, do ponto de vista experimental a equação (A.4) é uma relação que pode ser obtida por medida direta das propriedades do sistema, a dizer, $P, v, T$, as quais podem ser executadas com grande precisão ${ }^{14}$. Daí a partir da equação (A.4) pode-se escrever $P(v, T), v(P, T)$ e $T(P, v)$.

Ocorre porém, que não é possível obter completamente a entropia a partir da equação de estado (A.4) e, por extensão, o calor específico também não, de modo esta que não constitui uma equação fundamental [14]. Para determinar a entropia a equação (A.4) deve combinar-se com a equação experimental do calor específico, $c_{p}(T)$. Juntas, $F(P, v, T)$ e $c_{p}(T)$ constituem

13 Um obstáculo que dificulta o uso prático de $u(s, v)$, está presente no fato que a entropia não pode ser controlada e nem medida diretamente em laboratório. No sentido de controlá-la, isto é, mantê-la constante durante um experimento, seria necessário providenciar um recipiente ideal, adiabático e fechado e assegurar que o processo é reversível. No sentido de medí-la diretamente, seria necessário medir o fluxo de calor associado à mudança de temperatura, acordando com a equação $d s=d Q^{\circ} / T$, em um processo reversível. Neste caso, a escala de temperatura deve ser a absoluta; o uso de uma escala empírica (como a escala de temperatura dos gases-perfeitos) seria possível apenas quando fosse possível mostrar que ambas escalas são idênticas [14].

14 Tipicamente em laboratório pode-se facilmente medir $P\left(\epsilon_{p}=0.002 \%\right), v\left(\epsilon_{v}=0.01 \%\right)$ e $T$ da ordem de $0.001 K\left(\epsilon_{T}=0.0003 \%\right)[14]$. 
condição necessária e suficiente para estabelecer todas as propriedades termodinâmicas de uma substância pura, ou seja, fazem as vezes de uma equação fundamental. A prova dada a seguir considera somente processos isobáricos, o que não limita a generalidade dos resultados. No entanto, processos isocóricos são obtidos de maneira similar.

Das relações de Maxwell tem-se que

$$
\left(\frac{\partial s}{\partial P}\right)_{T}=-\left(\frac{\partial v}{\partial T}\right)_{P}=f_{1}(P, T)
$$

onde $f_{1}$ é uma função de estado. Integrando tem-se

$$
\int_{P *}^{P}\left(\frac{\partial s}{\partial P}\right)_{T} d P=\int_{P *}^{P} f_{1}(P, T) d P
$$

ou

$$
s(P, T)-s\left(P^{*}, T\right)=F_{1}(P, T)
$$

Note que o estado de referência denotado por $P^{*}$ corresponde à temperatura $T^{*}$ e ao volume $v^{*}$ de referência, de modo que

$$
F\left(P^{*}, v^{*}, T^{*}\right)=0
$$

é o ponto de referência da função de estado.

É claro que a constante de integração $s\left(P^{*}, T\right)$ não pode assumir valores arbitrários, pois a integral foi feita a uma temperatura $T$ constante que difere da temperatura do estado de referência $T^{*}$. Porém, se os valores arbitrários são denotados por $s\left(P^{*}, T^{*}\right)$, então é possível reescrever as diferenças como

$$
s(P, T)-s\left(P^{*}, T^{*}\right)-\left[s\left(P^{*}, T\right)-s\left(P^{*}, T^{*}\right)\right]=F_{1}(P, T)
$$

ou

$$
\begin{aligned}
s(P, T)-s\left(P^{*}, T^{*}\right) & =F_{1}(P, T)+\left[s\left(P^{*}, T\right)-s\left(P^{*}, T^{*}\right)\right] \\
& =F_{1}(P, T)+\phi_{1}(T)
\end{aligned}
$$


onde

$$
\phi_{1}(T)=s\left(P^{*}, T\right)-s\left(P^{*}, T^{*}\right)
$$

é uma função ainda desconhecida. Esta equação depende da temperatura e não pode ser obtida com a ajuda dos dados contidos na equação de estado. Ela requer um cálculo adicional das diferenças de entropia ao longo da isobárica de referência $P^{*}$. E, no intuito de realizar tais cálculos é necessário obter informação adicional de uma natureza empírica: fala-se em calor especifico.

O calor específico a pressão constante é definido como

$$
c_{P}=\left(\frac{\partial h}{\partial T}\right)_{P}
$$

porém o jacobiano permite reescrevê-la como

$$
c_{P}=\frac{\partial(h, P)}{\partial(T, P)} \frac{\partial(s, P)}{\partial(s, P)}=\left(\frac{\partial h}{\partial s}\right)_{P}\left(\frac{\partial T}{\partial s}\right)_{P}^{-1}
$$

ou

$$
c_{P}=T\left(\frac{\partial s}{\partial T}\right)_{P}
$$

Substituindo a equação (A.10) na equação acima resulta

$$
c_{P}=T \frac{\partial}{\partial T}\left[F_{1}(P, T)\right]_{P}+T \frac{\partial}{\partial T}\left[\phi_{1}(T)\right]_{P}
$$

ou, explicitando $F_{1}$,

$$
c_{P}(P, T)=-T \int_{P *}^{P}\left(\frac{\partial^{2} v}{\partial T^{2}}\right)_{P} d P+T\left(\frac{\partial \phi_{1}(T)}{\partial T}\right)_{P}
$$

Conhecendo $c_{P}$ ao longo da isóbara $P^{*}$ tem-se que

$$
c_{P}\left(P^{*}, T\right)=T\left(\frac{\partial \phi_{1}(T)}{\partial T}\right)_{P^{*}}
$$

Integrando resulta

$$
\phi_{1}(T)=\int_{T *}^{T} \frac{c_{P}\left(P^{*}, T\right)}{T} d T
$$

de modo que $\phi_{1}\left(T^{*}\right)=0$. Levando esta equação na (A.10) tem-se

$$
s(P, T)-s\left(P^{*}, T^{*}\right)=\int_{P *}^{P}\left(\frac{\partial v}{\partial T}\right)_{P} d P+\int_{T *}^{T} \frac{c_{P}\left(P^{*}, T\right)}{T} d T
$$


Isto demonstra que a entropia de uma substância pode ser completamente determinada se a equação de estado é conhecida a partir da experiência e se "em adição" o calor específico for conhecido como uma função da temperatura ao longo de uma isóbara. Ocorre que o calor específico não é completamente obtido através da equação de estado. Daí a necessidade de conhecê-lo experimentalmente. Na equação acima tem-se

$$
c_{p}(P, T)=-T \int_{P *}^{P}\left(\frac{\partial^{2} v}{\partial T^{2}}\right)_{P} d P+c_{P}\left(P^{*}, T\right)
$$

ou

$$
\left(\frac{\partial c_{P}}{\partial P}\right)_{T}=-T\left(\frac{\partial^{2} v}{\partial T^{2}}\right)_{P}
$$

o que mostra que a dependência do calor específico sobre a pressão é completamente determinado pela equação de estado, enquanto que sua completa dependência sobre a temperatura não o é.

\section{A.2 Das Equações de Estado de van der Waals e Peng-Robinson}

Equações de estado como a de van der Waals (EE-vdW) e a de Peng-Robinson (EE-PR) [25][32], representam suficientemente bem a continuidade de estados através do chamado loop de van der Waals. A equação de estado de van der Waals é dada por

$$
P=\frac{R T}{v-b_{c}}-\frac{a_{c}}{v^{2}}
$$

onde as constantes $a_{c}$ e $b_{c}$ são definidas em função do ponto crítico, $\Gamma_{c}$, conforme Tabela A.7. E a equação de estado de Peng-Robinson é dada por

$$
P=\frac{R T}{v-b_{c}}-\frac{a_{c}\left[1-\kappa\left(1-\sqrt{T / T_{c}}\right)\right]}{v^{2}+2 b_{c} v-b_{c}^{2}}
$$

onde as constantes $a_{c}$ e $b_{c}$ são definidas em função do ponto crítico, $\Gamma_{c}$, conforme Tabela A.7, $\kappa$ é dado por

$$
\kappa=0.37464+1.54226 \omega-0.26992 \omega^{2}
$$


onde no caso da água, $\omega=\omega_{H 2 O}=0.344$, é o fator acéntrico que compara a esfericidade da molécula de água em relação a uma molécula de gás esfericamente simétrica como o Argônio [32].

\begin{tabular}{|l|c|c|}
\cline { 2 - 3 } \multicolumn{1}{l|}{} & van de Waals & Peng-Robinson \\
\hline$a_{c}$ & $\frac{27}{64} \frac{\left(R T_{c}\right)^{2}}{P_{c}}$ & $0.45724 \frac{\left(R T_{c}\right)^{2}}{P_{c}}$ \\
\hline$b_{c}$ & $\frac{R T_{c}}{8 P_{c}}$ & $0.07780 \frac{R T_{c}}{P_{c}}$ \\
\hline
\end{tabular}

Tabela A.7- Constantes da equação de estados de van der Waals e de Peng-Robinson

Os cálculos do tamanho do núcleo crítico, $n^{*}$, via Teoria Entrópica da Nucleação (TEN), exigem o conhecimento da temperatura no espinodal, a qual é conhecida por intermédio do volume no estado. É importante saber qual das duas equações de estado melhor representa estado termodinâmico metaestável. Na Figura A.24 nota-se que loop de van der Waals no espinodal do líquido da EE-PR é mais profundo quando comparado com o do EE-vdW, o que significa que a pressão nesse ponto é menor em EE-PR.
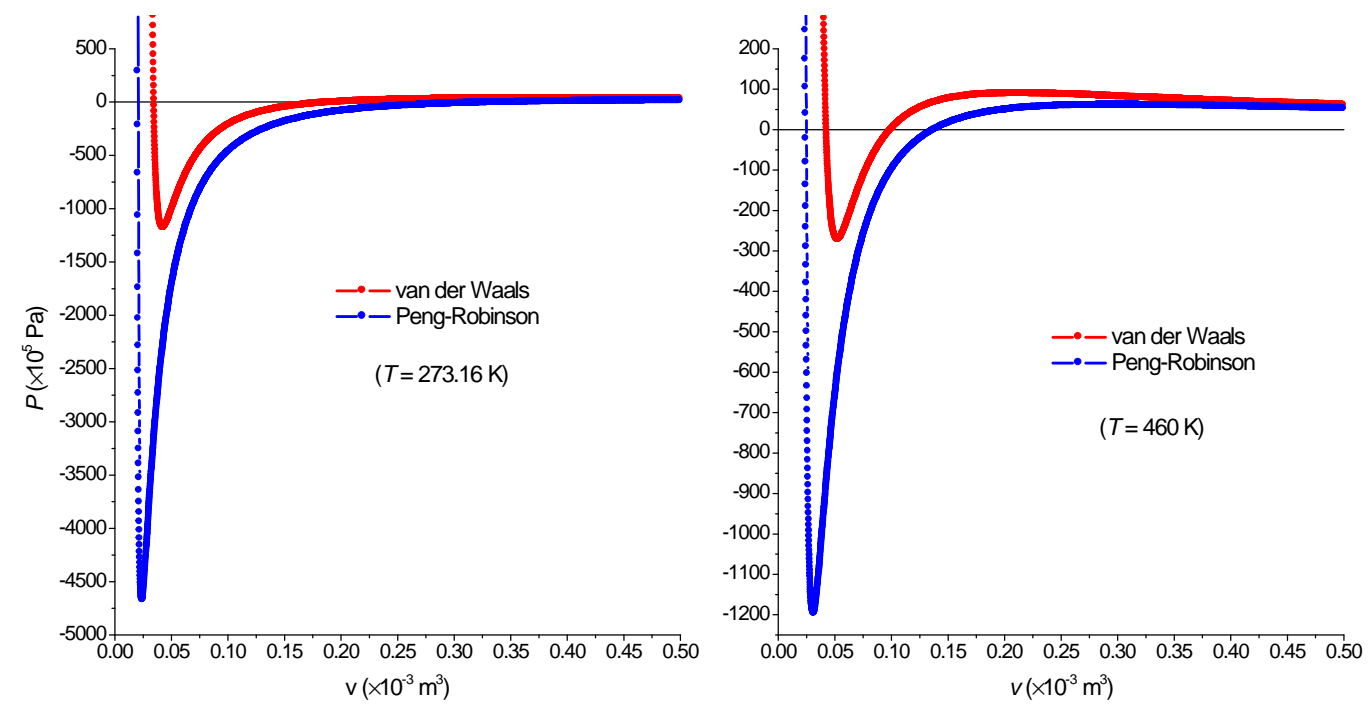

Fig. A.24- O loop de van der Waals no espinodal do líquido da EE-PR é mais profundo quando comparado com o do EE-vdW. 
As Figuras A.25, A.26, A.27 e A.28 ilustram o comportamento do volume teórico, eq. (A.22) e (A.23), em relação ao correspondente valor experimental $\left(v_{t e o} / v_{e x p}\right)$ obtidos no binodal, na região estável e na região metaestável do líquido, sendo que as variáveis pressão e/ou temperatura são experimentais. Para tanto, adotou-se os dados experimentais do livro de Joseph Kestin, A course in thermodynamics [14]. Para o binodal, foram utilizados 247 estados de onde se tem as medidas experimentais de temperatura, pressão, volume (do líquido, $v_{l}$, e do gás, $v_{g}$ ) entalpia (do líquido, $h_{l}$, e do gás, $h_{g}$ ) e entropia (do líquido, $s_{l}$, e do gás, $s_{g}$ ). Comparando o volume experimental com o teórico no binodal, verifica-se que a EE-PR é mais acurada que a EE-vdW em ambas as fases, além de mostrar-se mais comportada. Para a região da água líquida metaestável, os dados experimentais são creditados aos trabalhos de EvstefeevChukanov-Skripov [2][6]. Os gráficos na Figura A.28 indicam que a EE-PR é mais acurada também na região metaestável (à esquerda da pressão de equilíbrio termodinâmico, $P_{e}$ ).
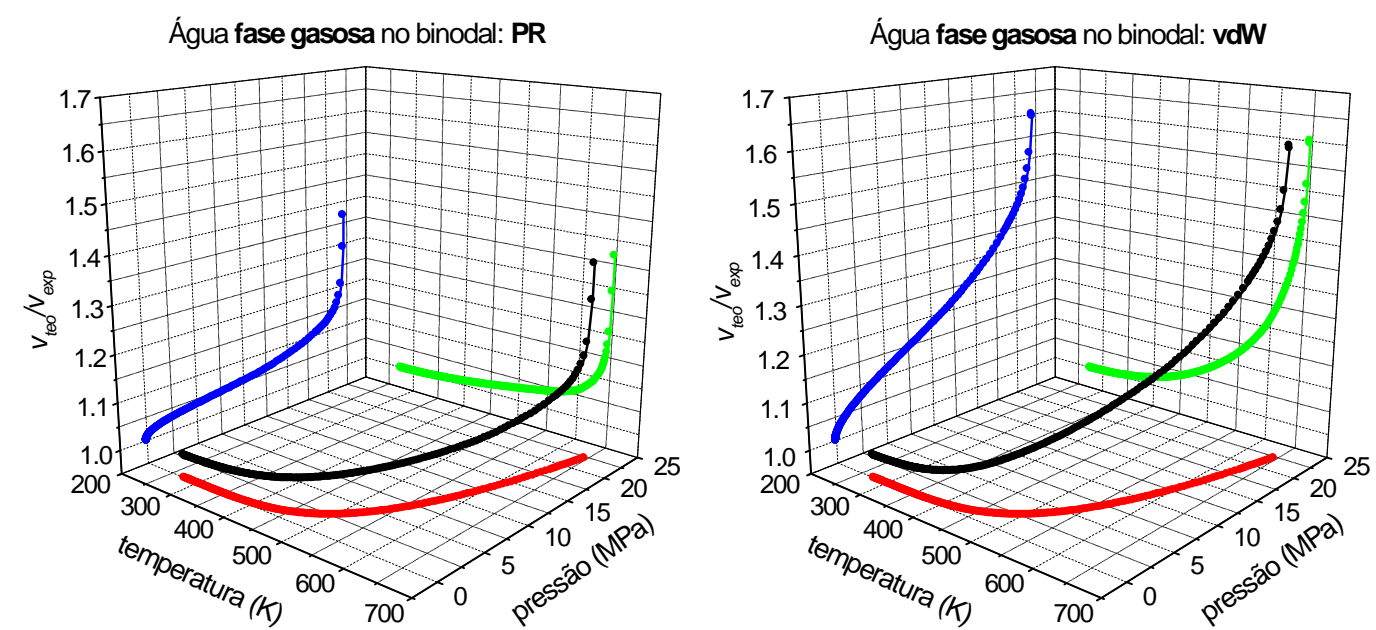

Fig. A.25- Curvas binodal da fase vapor da água desde o ponto triplo ao crítico. À direita tem-se a EE-vdW e à esquerda a EE-PR.

Para a fase líquida no binodal, a EE-vdW exibe resultados complexos a partir do ponto $\Gamma_{210}=(T, P)=\left(589.15 \mathrm{~K}, 1.0703 \times 10^{7} \mathrm{~Pa}\right)$. E para a EE-PR, o mesmo ocorre a partir de $\Gamma_{246}=(T, P)=\left(647.15 \mathrm{~K}, 2.2081 \times 10^{7} \mathrm{~Pa}\right)$, ou seja, o penúltimo ponto da seqüência. Essa 

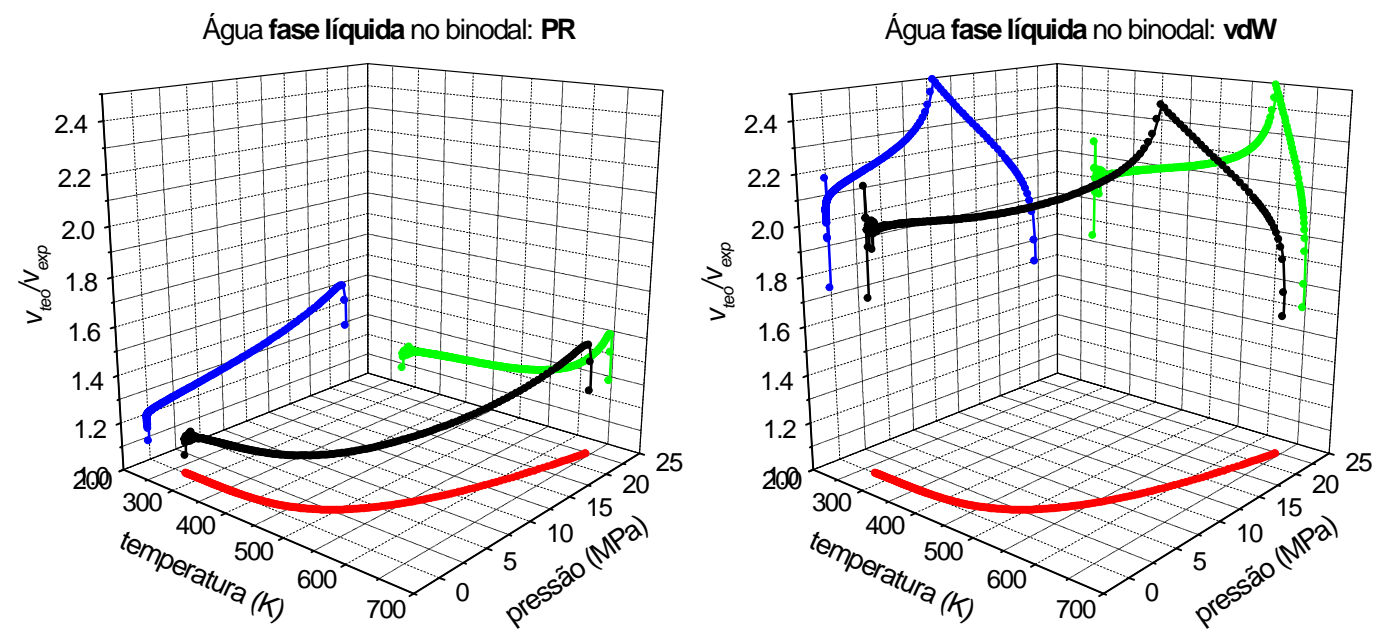

Fig. A.26- Curvas binodal da fase líquida da água desde o ponto triplo ao crítico. À direita tem-se a EE-vdW e à esquerda a EE-PR.

mudança no comportamento é reconhecida nos gráficos da Figura A.26 nos pontos onde sãos formados os bicos. Com relação à fase gasosa, não há formação de bicos e a acurácia da EE-PR também é superior. Cabe notar, entretanto, que ambas as funções desviam significativamente do experimental próximas ao ponto crítico.

Para investigar o comportamento das equações de estado nas regiões em que as fases líquida e gasosa são estáveis, também foram usados os dados experimentais apresentados por Kestin [14]. Neste caso a investigação dá-se à pressão constante. Na Figura A.27, a descontinuidade indica a passagem da fase líquida para a gasosa. Nela fica evidente a vocação das eq. (A.22) e (A.23) para a fase gasosa, principalmente a EE-vdW para pressões suficientemente longe da do ponto crítico. A seguir vem o estudo de EE-vdW e de EE-PR comparadas com os dados experimentais na região metaestável do líquido, Figura A.28. Nota-se que o desvio da EE-vdW aumenta nessa região, enquanto que a EE-PR mostra-se mais estável. 

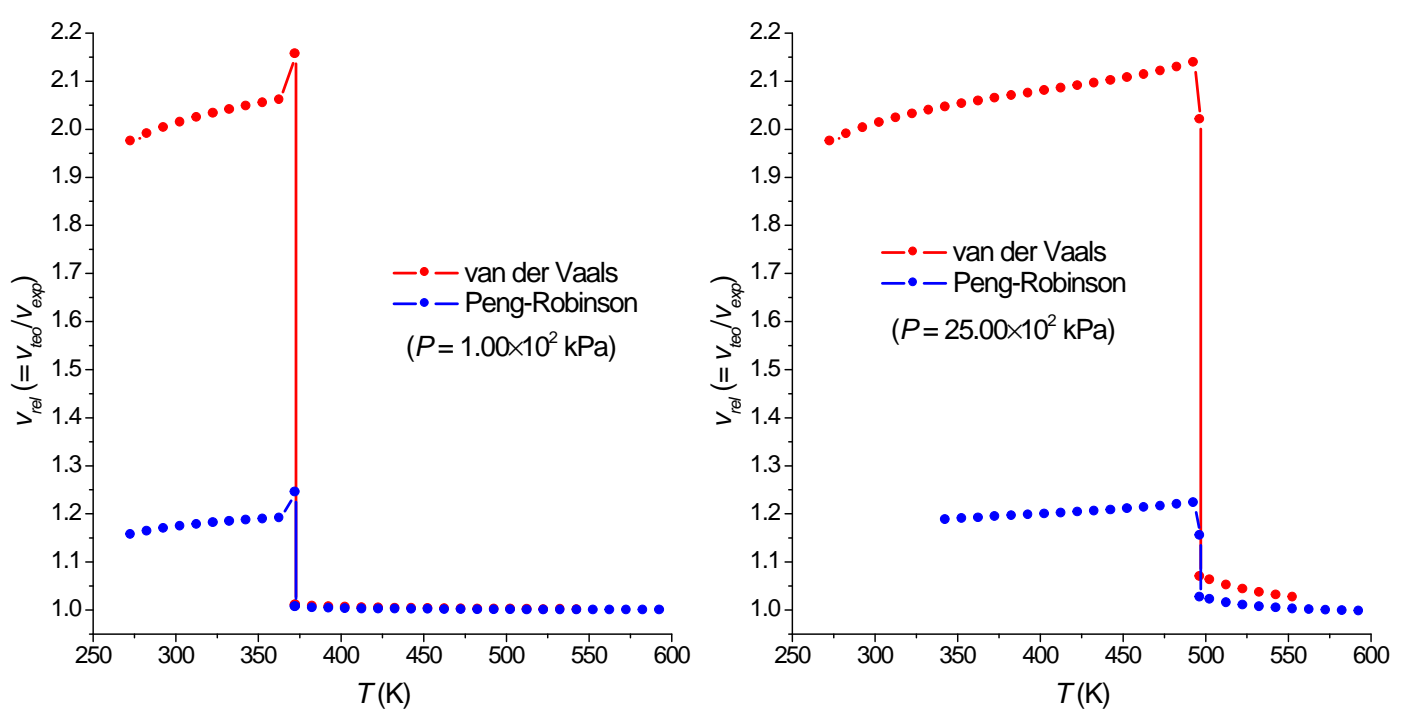

Fig. A.27- O desvio da EE-PR é significativo na fase líquida, enquanto que é muito grande o da EE-vdW.
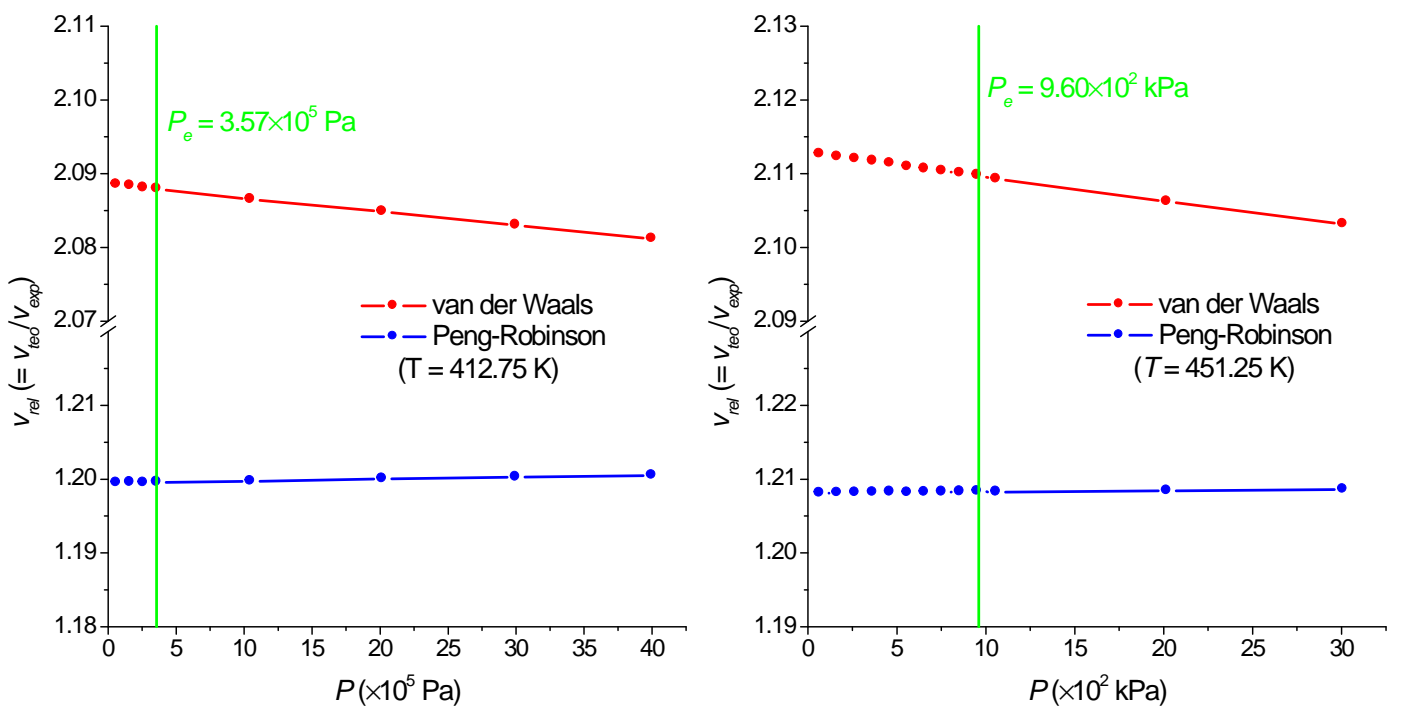

Fig. A.28- A falta de acurácia da EE-vdW mantém-se na região mestaestável. 


\section{B \\ Entropia de um Fluido Incompressível e de um Gás Perfeito}

O modelo do gás-perfeito representa as propriedades assintóticas de substâncias reais. Já o modelo de fluido-incompressível, usado em mecânica dos fluidos não apresenta caráter assintótico. Ele meramente representa a média de propriedades sobre um adequado e estreito intervalo de estados [14].

Entende-se por incompressível a taxa $\Delta v / \Delta P \simeq 0$ mesmo para $\Delta P>>0$. Em palavras, com a substância na fase líquida, em geral é necessário aplicar variações muito grandes na pressão para provocar alteração significativa no volume específico [14]. Neste caso o coeficiente de compressão isotérmica é bem pequeno,

$$
\beta=-\frac{1}{v}\left(\frac{\partial v}{\partial P}\right)_{T} \sim 0
$$

Em muitos problemas a variação da temperatura é pequena o suficiente para que seus efeitos sobre o volume específico também possam ser desconsiderados. No caso da água, mesmo com a temperatura variando de 0 a $100{ }^{\circ} \mathrm{C}$ à pressão de 1.01325 bar (1 atm), a variação do volume é cerca de $4 \%$. Isto significa que o coeficiente de expansão isotérmica também é bem pequeno

$$
\alpha=\frac{1}{v}\left(\frac{\partial v}{\partial T}\right)_{P} \sim 0
$$

Assim, pode-se assumir que para um fluido incompressível o volume é constante.

A diferença entre os calores específicos a volume constante e a pressão constante, resulta nulo

$$
c_{p}-c_{v}=v T \frac{\alpha^{2}}{\beta} \simeq 0
$$

ou, pode-se escrever, sem perda de generalidade,

$$
c_{v}=c_{77}=c_{l} .
$$


É fato experimental que a energia interna específica de um fluido incompressível é função somente da temperatura $T$ [14]. Sua variação em termos da equação (B.4) é escrita como

$$
d u=c_{l}(T) d T
$$

a qual integrada resulta

$$
u_{2}-u_{1}=c_{l}\left(T_{2}-T_{1}\right)
$$

onde admite-se $c_{l}=c t e$, pelas razões expostas acima.

A entalpia específica definida como

$$
h=u+P v
$$

tem a variação a volume constante dada por,

$$
h_{2}-h_{1}=u_{2}-u_{1}+v_{1}\left(P_{2}-P_{1}\right) \text {. }
$$

Admitindo, pelas razões expostas acima, que o calor específico é constante para o fluido na fase líquida, a equação (B.6) em (B.8) tem a forma

$$
h_{2}-h_{1}=c_{l}\left(T_{2}-T_{1}\right)+v_{1}\left(P_{2}-P_{1}\right) \text {. }
$$

A variação da entropia específica em função do volume específico e da temperatura é expressa por

$$
d s=\frac{c_{l}}{T} d T+\frac{\alpha}{\beta} d v
$$

ou, para um fluido incompressível,

$$
d s=\frac{c_{l}}{T} d T
$$

a qual é facilmente integrável, ou seja,

$$
s_{2}-s_{1}=c_{l} \ln \left(\frac{T_{2}}{T_{1}}\right)
$$

É notável que a entropia de um fluido incompressível (ou sólido) é função somente da temperatura. 
Resolvendo a equação (B.9) para $T_{2}$ e substituindo em (B.12) encontra-se a entropia de um fluido incompressível como função da entalpia e da pressão

$$
s_{2}-s_{1}=c_{l} \ln \left(\frac{\left(h_{2}-h_{1}\right)-v_{1}\left(P_{2}-P_{1}\right)}{c_{l} T_{1}}+1\right) \text {. }
$$

Para simplificar esta expressão, adota-se como estado de referência a vizinhança do ponto triplo $\left(P_{3}, v_{l 3}, T_{3}\right)$, de modo que $h_{1}=s_{1}=0$ [14]. Deste modo quando $P_{2}=P_{3}=$ cte a função passa pelo zero, $h_{2}=s_{2}=0$. Assim, abandonando o sub-índice 2 e substituindo o estado 1 como o do ponto triplo, pode-se reescrever esta expressão como

$$
s_{l}=c_{l} \ln \left(\frac{h-v_{l 3}\left(P-P_{3}\right)}{c_{l} T_{3}}+1\right) \text {. }
$$

Evidentemente que esta expressão é válida somente no domínio da fase líquida em estados estáveis. Esta expressão foi obtida considerando-se a igualdade em (B.4), a qual deixa de valer na região metaestável próxima ao espinodal, quando $c_{p}$ diverge e $c_{v}$ não.

Gases perfeitos não existem na natureza, mas as propriedades de todos os gases conhecidos aproximam-se daquelas dos gases ideais quando a pressão é extrapolada para zero. Para um gás ideal, a entropia como função da temperatura e da pressão é dada por

$$
d s=\frac{c_{P}^{\circ}}{T} d T-\frac{R}{P} d P
$$

onde $c_{P}^{\circ}$ refere-se ao calor específico a pressão constante de um gás ideal e, portanto, constante. Integrando do estado 1 até o estado 2 resulta

$$
s_{2}-s_{1}=c_{P}^{\circ}\left[\ln \left(\frac{T_{2}}{T_{1}}\right)-\frac{R}{c_{P}^{\circ}} \ln \left(\frac{P_{2}}{P_{1}}\right)\right]
$$

ou

$$
s_{2}-s_{1}=c_{P}^{\circ} \ln \left[\left(\frac{T_{2}}{T_{1}}\right)\left(\frac{P_{1}}{P_{2}}\right)^{\frac{R}{c_{P}^{\circ}}}\right] .
$$

A variação da entalpia para o gás ideal que varia seu estado isobaricamente tem a forma

$$
h_{2}-h_{1}=c_{P}^{\circ}\left(T_{2}-T_{1}\right)
$$


Resolvendo esta expressão para $T_{2}$ e levando-a em (B.17) tem-se

$$
s_{2}-s_{1}=c_{P}^{\circ} \ln \left[\left(\frac{h_{2}-h_{1}}{c_{P}^{\circ} T_{1}}+1\right)\left(\frac{P_{1}}{P_{2}}\right)^{\frac{R}{c_{P}^{\circ}}}\right] \text {. }
$$

Admitindo o mesmo estado de referência que em (B.14), porém para a fase gasosa, pode-se escrever

$$
s_{g}=s_{g 3}+c_{P}^{\circ} \ln \left[\left(\frac{h-h_{g 3}}{c_{P}^{\circ} T_{3}}+1\right)\left(\frac{P_{3}}{P}\right)^{\frac{R}{c_{P}^{\circ}}}\right] \text {, }
$$

onde $s_{g 3}$ e $h_{g 3}$ são a entropia e a entalpia de vaporização no ponto triplo, respectivamente. Deste modo, quando $P=P_{3}$, para $h=h_{g 3}$, tem-se $s=s_{g 3}$.

A Figura B.29 ilustra o comportamento das equações (B.14) e (B.20). Combinadas ambas as equações exibem os pontos binodal le e ge. Porém ambas não exibem os pontos espinodais.

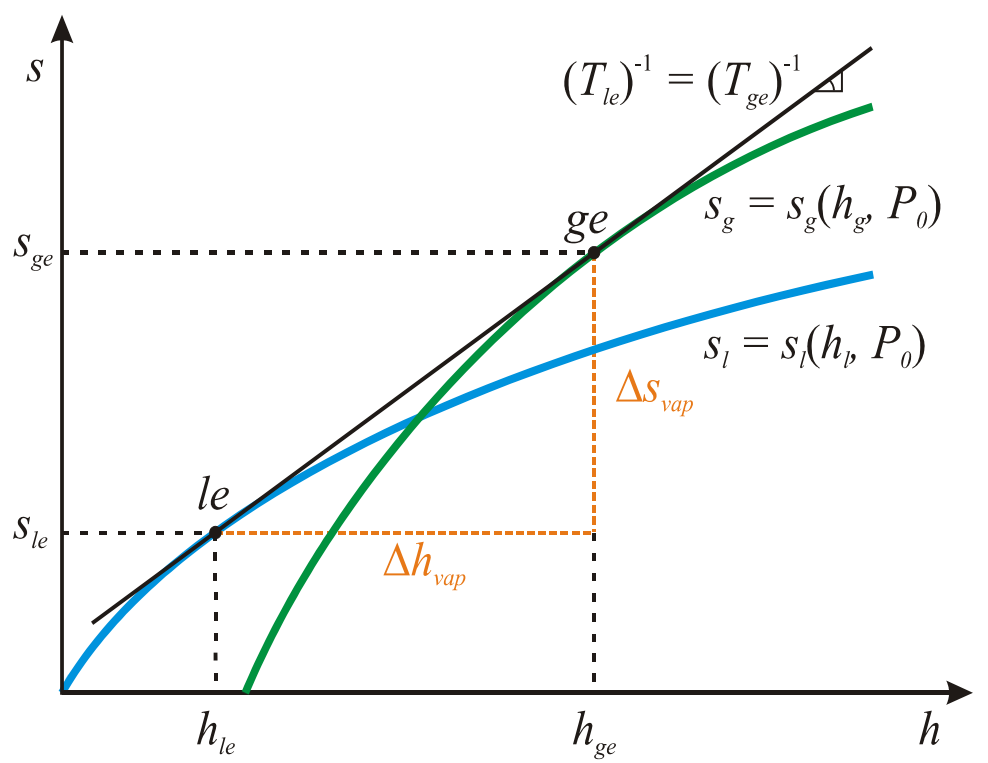

Fig. B.29- Entropia do líquido e do gás ideais não traz o limite de estabilidade da matéria. 


\section{C \\ Problema dos três números iguais}

Considere três números reais iguais, $a, b, c$. Expressa-se essa propriedade como

$$
a=b=c .
$$

Logicamente tal afirmação seria expressa por premissas como: se $a=b$ e $b=c$, então, $a=c$. Bastariam duas operações lógicas para se obter a terceira. Porém, em se tratando de números reais obtidos computacionalmente por diferentes caminhos o sinal de igualdade deve ser substituído pelo de aproximadamente igual e uma terceira operação lógica faz-se necessária, ou seja,

$$
a \simeq b \text { e } b \simeq c \text { e } a \simeq c
$$

Considerando a primeira premissa com alguma tolerância, $a \simeq b$, tem-se $b=a \epsilon_{b}$ onde $\epsilon_{b}$ $(\gtreqless 1)$ é o desvio, então

$$
\begin{aligned}
a & \simeq b \\
& \simeq a \epsilon_{b}
\end{aligned}
$$

ou

$$
\epsilon_{b} \gtreqless 1
$$

Evidentemente que se busca $\epsilon_{b}=1$, porém em se tratando de números reais obtidos numericamente (vide equações (D.11), (D.22) e (D.32)) este resultado exige precisão infinita o que torna proibitivo o custo computacional. A tolerância deve ser ajustada em acordo com o problema em estudo em que a igualdade é aplicada. A comparação entre os números é mais delicada com a segunda premissa, $b \simeq c$, ou seja, escrevendo $c=a \epsilon_{c}$, em que $\epsilon_{c}(\gtreqless 1)$ é o desvio

$$
a \epsilon_{b} \widetilde{81} a \epsilon_{c}
$$


ou

$$
\epsilon_{b} \gtreqless \epsilon_{c}
$$

onde verifica-se que o valor de $c$ pode desviar-se consideravelmente de $a$, pois o desvio de $c$ é comparado com o desvio de $b$ e não com a unidade. Esta situação é contornada com a imposição da premissa $a \simeq c$. Daí os três processos lógicos.

Busca-se uma função $f(a, b, c)=0$ que expressa a propriedade (C.1) em uma única expressão matemática e que seja computacionalmente estável. Uma solução encontrada é apresentada a seguir.

Considere os três números $a, b$ e $c$ expressos como vetores de um sistema cartesiano, ou seja, $\mathbf{a}=(a, 0,0), \mathbf{b}=(0, b, 0)$ e $\mathbf{c}=(0,0, c)$, conforme Figura C.30. Define-se sobre o plano $x y$ o vetor $r_{1}=\sqrt{a^{2}+b^{2}}$ que faz um ângulo $\theta_{1}=\tan ^{-1}\left(\frac{b}{a}\right)$ com o eixo- $x$; sobre o plano $y z$ o vetor $r_{2}=\sqrt{b^{2}+c^{2}}$ que faz um ângulo $\theta_{2}=\tan ^{-1}\left(\frac{c}{b}\right)$ com o eixo-y; e sobre o plano $z x$ o vetor $r_{3}=\sqrt{c^{2}+a^{2}}$ que faz um ângulo $\theta_{3}=\tan ^{-1}\left(\frac{a}{c}\right)$ com o eixo-z; e a diagonal do paralelepípedo $r=\sqrt{a^{2}+b^{2}+c^{2}}$.

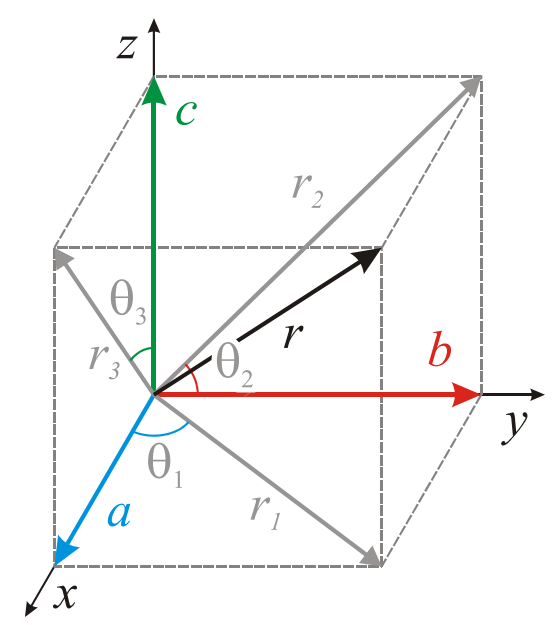

Fig. C.30- Representação vetorial dos três números iguais.

Sabe-se da geometria espacial que o paralelepípedo apresenta a seguinte propriedade

$$
r_{1}^{2}+r_{2}^{2}+r_{3}^{2}=2 r^{2}
$$


O quadrado do vetor $r_{1}$ é proporcional à área, $A_{1}=a b$, da face do paralelepípedo que se encontra sobre o plano $x y$. Escrevendo $a$ e $b$ em coordenadas polares tem-se

$$
\begin{aligned}
a b & =r_{1} \cos \theta_{1} \cdot r_{1} \sin \theta_{1} \\
& =r_{1}^{2} \cos \theta_{1} \sin \theta_{1}
\end{aligned}
$$

e como $\sin \left(2 \theta_{1}\right)=2 \cos \theta_{1} \sin \theta_{1}$, pode-se escrever

$$
r_{1}^{2}=\frac{2}{\sin \left(2 \theta_{1}\right)} a b
$$

Similarmente tem-se

$$
r_{2}^{2}=\frac{2}{\sin \left(2 \theta_{2}\right)} b c
$$

$\mathrm{e}$

$$
r_{3}^{2}=\frac{2}{\sin \left(2 \theta_{3}\right)} a c
$$

Substituindo as equações (C.11), (C.12) e (C.13) e a diagonal do paralelepípedo na equação (C.8) resulta

$$
\frac{a b}{\sin \left(2 \theta_{1}\right)}+\frac{b c}{\sin \left(2 \theta_{2}\right)}+\frac{a c}{\sin \left(2 \theta_{3}\right)}=a^{2}+b^{2}+c^{2}
$$

Esta igualdade é válida para quaisquer três números dispostos como vetores num sistema cartesiano. No caso especial, $a=b=c$, tem-se que $\theta_{1}=\theta_{2}=\theta_{3}=\pi / 4$ de modo que

$$
a^{2}+b^{2}+c^{2}=a b+b c+a c
$$

portanto um cubo. Porém em se tratando de números reais, em que $a \simeq b \simeq c \simeq a$, pode-se escrever, descartando as funções seno,

$$
a^{2}+b^{2}+c^{2} \geq a b+b c+a c
$$

ou

$$
(a+b+c)^{2} \geq 3(a b+b c+a c)
$$

Ora, isto permite escrever a função procurada

$$
f(a, b, c)=\frac{(a+b+c)^{2}}{a b+b c+a c}-3 \geq 0
$$


Cabe analisar a função dada em (C.18). Admitindo por simplicidade que $b=a x$, onde $x \in(0,2)$ e $c=a y, \operatorname{com} y \in(0,2)$, de modo que a equação (C.18) tem a forma

$$
f(a, b, c)=z=\frac{(1+x+y)^{2}}{x+x y+y}-3
$$

A Figura C.31 mostra a superfície da eq. (C.19) em que há um mínimo para $x=y=1$, quando se tem $a=b=c$. E portanto busca-se

$$
f_{\min }(a, b, c)=0
$$

com a propriedade

$$
d f=\left(\frac{\partial f}{\partial a}\right)_{b, c} d a+\left(\frac{\partial f}{\partial b}\right)_{a, c} d b+\left(\frac{\partial f}{\partial c}\right)_{a, b} d c=0
$$

ilustrada na Figura C.31

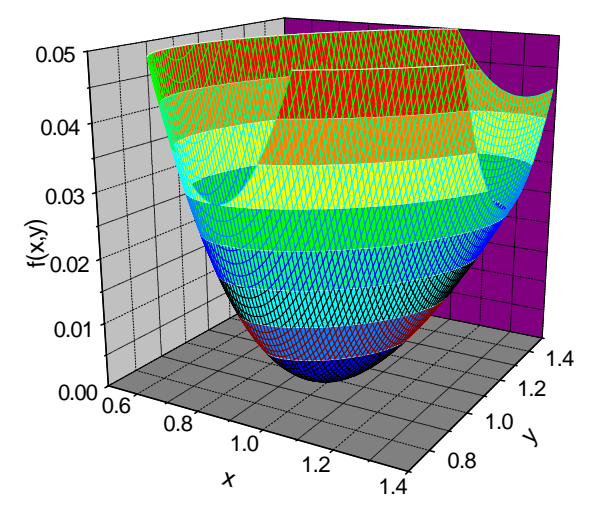

Fig. C.31- Superfície da eq. (C.20) tem a propriedade de ser mínima quando os três números são iguais.

Cabe notar que a eq. (C.18) ou a (C.20) são computacionalmente estáveis, inclusive em relação ao sinal algébrico dos três números 


\section{D \\ Função $s\left(h, P_{0}\right)$ alternativa}

\section{D.1 Sistema I}

A função $s\left(h, P_{0}\right)$ na forma polinomial permite a construção de um sistema de equações cuja $i$-ésima linha é dada por

$$
\sum_{j=1}^{8} h_{i}^{j+1} b_{j+1}^{(I)}=s_{i}-b_{0}^{(I)}-b_{1}^{(I)} h_{i}
$$

ou em notação matricial,

$$
\mathbf{M}^{(I)} \mathbf{b}^{(I)}=\mathbf{y}^{(I)}
$$

onde

$$
\mathbf{M}^{(I)}=\left[\begin{array}{cccc}
h_{1}^{2} & h_{1}^{3} & \cdots & h_{1}^{9} \\
h_{2}^{2} & h_{2}^{3} & \cdots & h_{2}^{9} \\
\vdots & \vdots & \ddots & \vdots \\
h_{8}^{2} & h_{8}^{3} & \cdots & h_{8}^{9}
\end{array}\right], \mathbf{b}^{(I)}=\left[\begin{array}{c}
b_{2}^{(I)} \\
b_{3}^{(I)} \\
\vdots \\
b_{9}^{(I)}
\end{array}\right] \text { e } \mathbf{y}^{(I)}=\left[\begin{array}{c}
s_{1}-b_{0}^{(I)}-b_{1}^{(I)} h_{1} \\
s_{2}-b_{0}^{(I)}-b_{1}^{(I)} h_{2} \\
\vdots \\
s_{8}-b_{0}^{(I)}-b_{1}^{(I)} h_{8}
\end{array}\right]
$$

O coeficientes $b_{k+1}^{(I)}$, para $k=1,2, \ldots, 8$, podem ser calculados pela regra de Cramer

$$
b_{k+1}^{(I)}=\frac{\left|\mathbf{N}_{k}^{(I)}\right|}{\left|\mathbf{M}^{(I)}\right|} \quad(k=1,2, \ldots, 8)
$$

onde

$$
\left|\mathbf{N}_{k}^{(I)}\right|=\operatorname{det}\left[\begin{array}{cccccc}
h_{1}^{2} & h_{1}^{3} & \cdots & y_{1}^{(I)} & \cdots & h_{1}^{9} \\
h_{2}^{2} & h_{2}^{3} & \cdots & y_{2}^{(I)} & \cdots & h_{2}^{9} \\
\vdots & \vdots & \ddots & \vdots & \ddots & \vdots \\
h_{8}^{2} & h_{8}^{3} & \cdots & \overbrace{c o l}^{y_{8}^{(I)}} & \cdots & h_{8}^{9} \\
& & & &
\end{array}\right]
$$

ou seja, o cálculo de $\left|\mathbf{N}_{k}^{(I)}\right|$ exige que a $k$-ésima coluna de $\mathbf{M}^{(I)}$ seja substituída pelo vetor coluna $\mathbf{y}^{(I)}$. Essa construção permite alguma simplificação na obtenção do determinante. Cada 
$i$-ésima linha de $\mathbf{M}^{(I)}$ é multiplicada por $h_{i}^{2}$, de modo que estes podem ser fatorados ${ }^{15}$

$$
\left|\mathbf{M}^{(I)}\right|=h_{1}^{2} \cdot h_{2}^{2} \cdots h_{8}^{2} \cdot \operatorname{det}\left[\begin{array}{cccc}
1 & h_{1} & \cdots & h_{1}^{7} \\
1 & h_{2} & \cdots & h_{2}^{7} \\
\vdots & \vdots & \ddots & \vdots \\
1 & h_{8} & \cdots & h_{8}^{7}
\end{array}\right]
$$

ou

$$
\left|\mathbf{M}^{(I)}\right|=\prod_{i=1}^{8} h_{i}^{2} \cdot|\mathbf{M}|
$$

onde

$$
|\mathbf{M}|=\operatorname{det}\left[\begin{array}{cccc}
1 & h_{1} & \cdots & h_{1}^{7} \\
1 & h_{2} & \cdots & h_{2}^{7} \\
\vdots & \vdots & \ddots & \vdots \\
1 & h_{8} & \cdots & h_{8}^{7}
\end{array}\right]
$$

Com alguma adequação, o determinante no numerador de (D.4) também pode ser fatorado

$$
\left|\mathbf{N}_{k}^{(I)}\right|=\prod_{i=1}^{8} h_{i}^{2} \cdot\left|\widetilde{\mathbf{N}}_{k}^{(I)}\right|
$$

onde

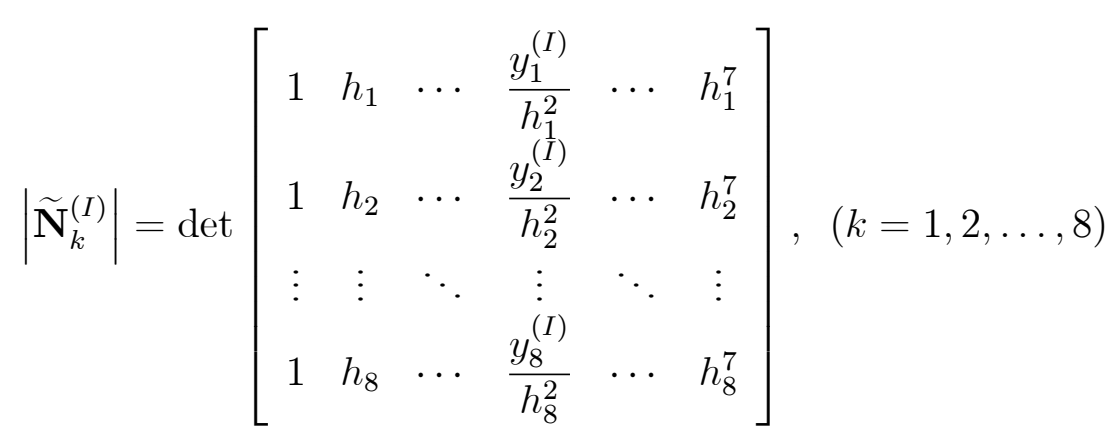

Assim, substituindo as equações (D.7) e (D.9) em (D.4) resulta

$$
b_{k+1}^{(I)}=\frac{\left|\widetilde{\mathbf{N}}_{k}^{(I)}\right|}{|\mathbf{M}|}
$$

15 Da Álgebra Linear tem-se o seguinte teorema sobre a propriedade dos determinantes: Suponha a matriz B obtida de A por uma operação elementar de linha (coluna), então, multiplicando-se uma linha (coluna) de A por um escalar k, tem-se, $|\mathbf{B}|=k|\mathbf{A}|[8]$. 


\section{D.2 Sistema II}

O sistema II é construído a partir do sistema I onde cada linha é obtida pela derivada em relação a $h_{i}$, ou seja

$$
\sum_{j=1}^{8}(j+1) h_{i}^{j} b_{j+1}^{(I I)}=\frac{1}{T_{i}}-b_{1}^{(I I)}
$$

e em notação matricial,

$$
\mathbf{M}^{(I I)} \mathbf{b}^{(I I)}=\mathbf{y}^{(I I)}
$$

onde

$$
\mathbf{M}^{(I I)}=\left[\begin{array}{cccc}
2 h_{1} & 3 h_{1}^{2} & \cdots & 9 h_{1}^{8} \\
2 h_{2} & 3 h_{2}^{2} & \cdots & 9 h_{2}^{8} \\
\vdots & \vdots & \ddots & \vdots \\
2 h_{8} & 3 h_{8}^{2} & \cdots & 9 h_{8}^{8}
\end{array}\right], \mathbf{b}^{(I I)}=\left[\begin{array}{c}
b_{2}^{(I I)} \\
b_{3}^{(I I)} \\
\vdots \\
b_{9}^{(I I)}
\end{array}\right] \text { e } \mathbf{y}^{(I I)}=\left[\begin{array}{c}
1 / T_{1}-b_{1}^{(I I)} \\
1 / T_{2}-b_{1}^{(I I)} \\
\vdots \\
1 / T_{8}-b_{1}^{(I I)}
\end{array}\right]
$$

Similarmente à equação (D.11), os coeficientes $b_{k+1}^{(I I)}$, para $k=1,2, \ldots, 8$, são calculados pela regra de Cramer

$$
b_{k+1}^{(I I)}=\frac{\left|\mathbf{N}_{k}^{(I I)}\right|}{\left|\mathbf{M}^{(I I)}\right|}
$$

onde

$$
\mathbf{N}_{k}^{(I I)}=\left[\begin{array}{cccccc}
2 h_{1} & 3 h_{1}^{2} & \cdots & y_{1}^{(I I)} & \cdots & 9 h_{1}^{8} \\
2 h_{2} & 3 h_{2}^{2} & \cdots & y_{2}^{(I I)} & \cdots & 9 h_{2}^{8} \\
\vdots & \vdots & \ddots & \vdots & \ddots & \vdots \\
2 h_{8} & 3 h_{8}^{2} & \cdots & \overbrace{\mathrm{col}=k}^{(I I)} & \cdots & 9 h_{8}^{8}
\end{array}\right], \quad(k=1,2, \ldots, 8)
$$

Além de cada $i$-ésima linha apresentar um fator comum, $h_{i}$, cada coluna uma constante $(k+1)$, para $k=1,2, \ldots, 8$. A fatoração resulta

$$
\left|\mathbf{M}^{(I I)}\right|=(2 \cdot 3 \cdots 9)\left(h_{1} \cdot h_{2} \cdots \cdots h_{9}\right) \cdot \operatorname{det}\left[\begin{array}{cccc}
1 & h_{1} & \cdots & h_{1}^{7} \\
1 & h_{2} & \cdots & h_{2}^{7} \\
\vdots & \vdots & \ddots & \vdots \\
1 & h_{8} & \cdots & h_{8}^{7}
\end{array}\right]
$$

ou

$$
\left|\mathbf{M}^{(I I)}\right|=9 ! \cdot \prod_{i=1}^{8} h_{i} \cdot|\mathbf{M}|
$$


onde $|\mathbf{M}|$ é dado pela equação (D.8). O numerador também admite alguma fatoração. Escrevendo

$$
\left|\mathbf{N}_{k}^{(I I)}\right|=\operatorname{det}\left[\begin{array}{cccccc}
2 h_{1} & 3 h_{1}^{2} & \cdots & \frac{(k+1) h_{1}}{(k+1) h_{1}} y_{1}^{(I I)} & \ldots & 9 h_{1}^{8} \\
2 h_{2} & 3 h_{2}^{2} & \cdots & \frac{(k+1) h_{2}}{(k+1) h_{2}} y_{2}^{(I I)} & \cdots & 9 h_{2}^{8} \\
\vdots & \vdots & \ddots & \vdots & \ddots & \vdots \\
2 h_{8} & 3 h_{8}^{2} & \cdots & \frac{(k+1) h_{8}}{(k+1) h_{8}} y_{8}^{(I I)} & \cdots & 9 h_{8}^{8}
\end{array}\right], \quad(k=1,2, \ldots, 8)
$$

tem-se a fatoração resulta

$$
\left|\mathbf{N}_{k}^{(I I)}\right|=\frac{9 !}{(k+1)} \cdot \prod_{i=1}^{8} h_{i} \cdot\left|\widetilde{\mathbf{N}}_{k}^{(I I)}\right|
$$

onde

$$
\left|\widetilde{\mathbf{N}}_{k}^{(I I)}\right|=\operatorname{det}\left[\begin{array}{cccccc}
1 & h_{1} & \cdots & \frac{y_{1}^{(I I)}}{h_{1}} & \cdots & h_{1}^{7} \\
1 & h_{2} & \cdots & \frac{y_{2}^{(I I)}}{h_{2}} & \cdots & h_{2}^{7} \\
\vdots & \vdots & \ddots & \vdots & \ddots & \vdots \\
1 & h_{8} & \cdots & \frac{y_{8}^{(I I)}}{h_{8}} & \cdots & h_{8}^{7}
\end{array}\right],(k=1,2, \ldots, 8)
$$

A constante $(k+1)$ tem o papel de permitir escrever 9!. Assim, levando as equações (D.18) e (D.20) em (D.15) resulta

$$
b_{k+1}^{(I I)}=\frac{\left|\widetilde{\mathbf{N}}_{k}^{(I I)}\right|}{(k+1)|\mathbf{M}|}
$$

onde os determinantes $|\mathbf{M}|$ nas equações (D.11) e (D.22) são os mesmos.

\section{D.3 Sistema III}

O sistema III é construído a partir do sistema II, no qual cada $i$-ésima linha é construída a partir da derivada em relação a $h_{i}$, ou seja,

$$
\sum_{j=1}^{8} j(j+1) h_{i}^{j-1} b_{j+1}^{(I I)}=-\frac{1}{T_{i}^{2} c_{p, i}}
$$

Em notação matricial verifica-se

$$
\mathbf{M}^{(I I I)} \mathbf{b}^{(I I I)}=\mathbf{y}^{(I I I)}
$$


onde

$$
\mathbf{M}^{(I I I)}=\left[\begin{array}{cccc}
2 & 2 \cdot 3 \cdot h_{1} & \cdots & 8 \cdot 9 \cdot h_{1}^{7} \\
2 & 2 \cdot 3 \cdot h_{2} & \cdots & 8 \cdot 9 \cdot h_{2}^{7} \\
\vdots & \vdots & \ddots & \vdots \\
2 & 2 \cdot 3 \cdot h_{8} & \cdots & 8 \cdot 9 \cdot h_{8}^{7}
\end{array}\right], \mathbf{b}^{(I I I)}=\left[\begin{array}{c}
b_{2}^{(I I I)} \\
b_{3}^{(I I I)} \\
\vdots \\
b_{9}^{(I I I)}
\end{array}\right] \quad \text { e } \mathbf{y}^{(I I I)}=\left[\begin{array}{c}
-\left[T_{1}^{2} c_{p, 1}\right]^{-1} \\
-\left[T_{2}^{2} c_{p, 2}\right]^{-1} \\
\vdots \\
-\left[T_{8}^{2} c_{p, 8}\right]^{-1}
\end{array}\right]
$$

Os coeficientes $b_{k+1}^{(I I I)}$, para $k=1,2, \ldots, 8$, podem ser calculados pela regra de Cramer

$$
b_{k+1}^{(I I I)}=\frac{\left|\mathbf{N}_{k}^{(I I I)}\right|}{\left|\mathbf{M}^{(I I I)}\right|}
$$

onde

$$
\mathbf{N}_{k}^{(I I I)}=\left[\begin{array}{cccccc}
2 & 2 \cdot 3 \cdot h_{1} & \cdots & \frac{k(k+1)}{k(k+1)} y_{1}^{(I I I)} & \cdots & 8 \cdot 9 \cdot h_{1}^{7} \\
2 & 2 \cdot 3 \cdot h_{2} & \cdots & \frac{k(k+1)}{k(k+1)} y_{2}^{(I I I)} & \cdots & 8 \cdot 9 \cdot h_{2}^{7} \\
\vdots & \vdots & \ddots & \vdots & \ddots & \vdots \\
2 & 2 \cdot 3 \cdot h_{8} & \cdots & \frac{k(k+1)}{k(k+1)} y_{8}^{(I I I)} & \cdots & 8 \cdot 9 \cdot h_{8}^{7}
\end{array}\right], \quad(k=1,2, \ldots, 8)
$$

Os determinantes em (D.26) podem ser fatorados como segue

$$
\left|\mathbf{M}^{(I I I)}\right|=2 \cdot(2 \cdot 3) \cdots(8 \cdot 9) \cdot \operatorname{det}\left[\begin{array}{cccc}
1 & h_{1} & \cdots & h_{1}^{7} \\
1 & h_{2} & \cdots & h_{2}^{7} \\
\vdots & \vdots & \ddots & \vdots \\
1 & h_{8} & \cdots & h_{8}^{7}
\end{array}\right]
$$

$\mathrm{ou}$

$$
\left|\mathbf{M}^{(I I I)}\right|=8 ! \cdot 9 ! \cdot|\mathbf{M}|
$$

onde $|\mathbf{M}|$ é dado pela equação (D.8). E também,

$$
\left|\mathbf{N}_{k}^{(I I I)}\right|=\frac{8 ! \cdot 9 !}{k(k+1)} \cdot\left|\widetilde{\mathbf{N}}_{k}^{(I I I)}\right|
$$

onde

$$
\left|\widetilde{\mathbf{N}}_{k}^{(I I I)}\right|=\operatorname{det}\left[\begin{array}{cccccc}
1 & h_{1} & \cdots & y_{1}^{(I I I)} & \cdots & h_{1}^{7} \\
1 & h_{2} & \cdots & y_{2}^{(I I I)} & \cdots & h_{2}^{7} \\
\vdots & \vdots & \ddots & \vdots & \ddots & \vdots \\
1 & h_{8} & \cdots & y_{8}^{(I I I)} & \cdots & h_{8}^{7}
\end{array}\right],(k=1,2, \ldots, 8)
$$

Assim, levando as equações (D.29) e (D.30) na (D.26) resulta

$$
b_{k+1}^{(I I I)}=\frac{\left|\widetilde{\mathbf{N}}_{k}^{(I I I)}\right|}{k(k+1)|\mathbf{M}|}
$$




\section{D.4 Novo sistema}

Por construção tem-se que os coeficientes dos três sistemas são iguais, ou seja, $b_{1}^{(I)}=b_{1}^{(I I)} \mathrm{e}$ $b_{k+1}^{(I)}=b_{k+1}^{(I I)}=b_{k+1}^{(I I I)}$ para $k=1,2, \ldots, 8$. Na verdade são aproximadamente iguais, o que remete ao problema dos três números iguais (vide Apêndice C). Assim com a equação (C.18) tem-se a desigualdade

$$
\left(b_{k+1}^{(I)}+b_{k+1}^{(I I)}+b_{k+1}^{(I I I)}\right)^{2} \geq 3\left(b_{k+1}^{(I)} \cdot b_{k+1}^{(I I)}+b_{k+1}^{(I)} \cdot b_{k+1}^{(I I I)}+b_{k+1}^{(I I)} \cdot b_{k+1}^{(I I I)}\right)
$$

para $k=1,2, \ldots, 8$. Usando as equações (D.11), (D.22) e (D.32)

$$
\begin{aligned}
& \left\{\frac{\left|\widetilde{\mathbf{N}}_{k}^{(I)}\right|}{|\mathbf{M}|}+\frac{\left|\tilde{\mathbf{N}}_{k}^{(I I)}\right|}{(k+1)|\mathbf{M}|}+\frac{\left|\widetilde{\mathbf{N}}_{k}^{(I I I)}\right|}{k(k+1)|\mathbf{M}|}\right\}^{2} \\
\geq & 3\left\{\frac{\left|\widetilde{\mathbf{N}}_{k}^{(I)}\right|}{|\mathbf{M}|} \cdot \frac{\left|\tilde{\mathbf{N}}_{k}^{(I I)}\right|}{(k+1)|\mathbf{M}|}+\frac{\left|\tilde{\mathbf{N}}_{k}^{(I)}\right|}{|\mathbf{M}|} \cdot \frac{\left|\tilde{\mathbf{N}}_{k}^{(I I I)}\right|}{k(k+1)|\mathbf{M}|}+\frac{\left|\widetilde{\mathbf{N}}_{k}^{(I I)}\right|}{(k+1)|\mathbf{M}|} \cdot \frac{\left|\widetilde{\mathbf{N}}_{k}^{(I I I)}\right|}{k(k+1)|\mathbf{M}|}\right. \text { (D.85) }
\end{aligned}
$$

Este arranjo permite alguma simplificação,

$$
\begin{aligned}
& \left\{k(k+1)\left|\widetilde{\mathbf{N}}_{k}^{(I)}\right|+k\left|\widetilde{\mathbf{N}}_{k}^{(I I)}\right|+\left|\widetilde{\mathbf{N}}_{k}^{(I I I)}\right|\right\}^{2} \\
\geq & \left.3 k\left\{k(k+1)\left|\widetilde{\mathbf{N}}_{k}^{(I)}\right| \cdot\left|\widetilde{\mathbf{N}}_{k}^{(I I)}\right|+(k+1)\left|\widetilde{\mathbf{N}}_{k}^{(I)}\right| \cdot\left|\widetilde{\mathbf{N}}_{k}^{(I I I)}\right|+\left|\widetilde{\mathbf{N}}_{k}^{(I I)}\right| \cdot\left|\widetilde{\mathbf{N}}_{k}^{(I I I)}\right|\right\} \mathrm{D} .36\right)
\end{aligned}
$$

Finalmente tem-se

$$
\frac{\left\{k(k+1)\left|\widetilde{\mathbf{N}}_{k}^{(I)}\right|+k\left|\widetilde{\mathbf{N}}_{k}^{(I I)}\right|+\left|\widetilde{\mathbf{N}}_{k}^{(I I I)}\right|\right\}^{2}}{k(k+1)\left|\widetilde{\mathbf{N}}_{k}^{(I)}\right| \cdot\left|\widetilde{\mathbf{N}}_{k}^{(I I)}\right|+(k+1)\left|\widetilde{\mathbf{N}}_{k}^{(I)}\right| \cdot\left|\widetilde{\mathbf{N}}_{k}^{(I I I)}\right|+\left|\widetilde{\mathbf{N}}_{k}^{(I I)}\right| \cdot\left|\widetilde{\mathbf{N}}_{k}^{(I I I)}\right|}-3 k \geq 0
$$

o que permite escrever a função

$$
f_{k, \min }\left(c_{0}, c_{1}, h_{r l s}, s_{r l s}, T_{r l s}, h_{r g s}, s_{r g s}, T_{r g s}\right)=0, \quad(k=1,2, \ldots, 8)
$$

onde $c_{0}=b_{0}^{(I)} / s_{3}$ e $c_{1}=b_{1}^{(I)} h_{3} / s_{3}$ e assim obter um sistema não-linear na forma reduzida com oito linhas e oito incógnitas. 


\section{$\mathbf{E}$ \\ Teoria da Flutuação (Landau)}

\section{E.1 Temperatura}

A energia média do sistema é dada por [30]

$$
\left\langle E_{j}\right\rangle=\frac{\sum_{j} E_{j} \exp \left(-\theta E_{j}\right)}{\sum_{j} \exp \left(-\theta E_{j}\right)}=-\frac{\partial}{\partial \theta} \ln Z,
$$

onde $Z$ é a função de partição, a qual no limite termodinâmico é dada por

$$
Z=\sum_{j} \exp \left(-\theta E_{j}\right)
$$

na qual $j$ é o $j$-ésimo estado disponível do sistema e $\theta=\frac{1}{k_{B} T}$ com $k_{B}$ sendo a constante de Boltzmann e $T$ a temperatura.

O desvio quadrático ou flutuação quadrática média é dado por [30]

$$
\begin{aligned}
\left\langle\left(\Delta E_{j}\right)^{2}\right\rangle & =\left\langle\left(E_{j}-\left\langle E_{j}\right\rangle\right)^{2}\right\rangle=\left\langle E_{j}^{2}\right\rangle-\left\langle E_{j}\right\rangle^{2} \\
& =\frac{\partial}{\partial \theta}\left[\frac{1}{Z} \frac{\partial Z}{\partial \theta}\right]=-\frac{\partial}{\partial \theta}\left\langle E_{j}\right\rangle
\end{aligned}
$$

Identificando o valor esperado da energia do sistema com a energia interna média, $\left\langle E_{j}\right\rangle \equiv$ $\langle U\rangle$,

$$
\left\langle(\Delta U)^{2}\right\rangle=-\frac{\partial}{\partial \theta}\langle U\rangle=k_{B} T^{2} \frac{\partial U}{\partial T}
$$

ou

$$
\left\langle(\Delta U)^{2}\right\rangle=n_{m} k_{B} T^{2} C_{V}
$$

onde $\left(\frac{\partial U}{\partial T}\right)_{V, n_{m}}=n_{m} C_{V}$, visto que $U=U\left(V, T, n_{m}\right)$ onde $n_{m}$ é o número de moles e $C_{v}$ é a capacidade térmica molar. Porém, a diferença finita da energia interna dada por

$$
\Delta U=n_{91} C_{V} \Delta T
$$


substituída acima resulta

$$
\left\langle\left(n_{m} C_{V} \Delta T\right)^{2}\right\rangle \cong n_{m} C_{V}\left\langle(\Delta T)^{2}\right\rangle=n_{m} k_{B} T^{2} C_{V}
$$

Assim, o desvio quadrático médio da temperatura é escrito como

$$
\left\langle(\Delta T)^{2}\right\rangle=\frac{k_{B} T^{2}}{n_{m} C_{V}}
$$

ou

$$
\left\langle(\Delta T)^{2}\right\rangle=\frac{R T^{2}}{n C_{V}}
$$

dado que $n_{m}=n / N_{A}$, onde $N_{A}$ é o número de Avogadro, então

Agora, o sistema proposto tem o estado descrito por duas variáveis independentes, a entalpia $H$ e a pressão $P$. Assim, a energia interna do sistema pode ser expressa como

$$
U(H, P)=H-P V(H, P)
$$

Dessa forma a diferencial da energia interna em relação à temperatura, tomada a pressão constante é expressa como

$$
\left(\frac{\partial U}{\partial T}\right)_{P}=\left(\frac{d U}{d H}\right)_{P}\left(\frac{d H}{d T}\right)_{P}=n_{m} C_{P}\left(\frac{d U}{d H}\right)_{P}
$$

onde $n_{m}$ é o número de moles e $C_{P}=C_{P}(H, P)$ é a capacidade térmica molar a pressão constante. Usando a definição da energia interna dada acima, tem-se

$$
\left(\frac{\partial U}{\partial T}\right)_{P}=n_{m} C_{P}\left[1-P\left(\frac{\partial V}{\partial H}\right)_{P}\right]
$$


onde $V=\left(\frac{\partial H}{\partial P}\right)_{S}$ é o volume específico molar ${ }^{16}$. Buscando a flutuação da temperatura, pode-se escrever

$$
\Delta U=n_{m} C_{P}\left[1-P\left(\frac{\partial V}{\partial H}\right)_{P}\right] \Delta T
$$

a qual substituída na equação (E.5) resulta

$$
\left\langle\left(n_{m} C_{P}\left[1-P\left(\frac{\partial V}{\partial H}\right)_{P}\right] \Delta T\right)^{2}\right\rangle=n_{m} k_{B} T^{2} C_{P}\left[1-P\left(\frac{\partial V}{\partial H}\right)_{P}\right]
$$

Assumindo que $\left\langle\left(C_{P}\left[1-P\left(\frac{\partial V}{\partial H}\right)_{P}\right]\right)^{2}\right\rangle \simeq\left(C_{P}\left[1-P\left(\frac{\partial V}{\partial H}\right)_{P}\right]\right)^{2}$ tem-se

$$
\left\langle(\Delta T)^{2}\right\rangle=\frac{k_{B} T^{2}}{n_{m} C_{P}\left[1-P\left(\frac{\partial V}{\partial H}\right)_{P}\right]}
$$

como $n_{m}=n / N_{A}$ e $N_{A} k_{B}=R$ resulta que a flutuação da temperatura é dada por

$$
\left\langle(\Delta T)^{2}\right\rangle=\frac{R T^{2}}{n C_{P}\left[1-P\left(\frac{\partial V}{\partial H}\right)_{P}\right]}
$$

Note que em (E.9) $c_{P}$ é o calor específico a pressão constante, de modo que a constante dos gases é dada por $R=0.461526 \mathrm{~kJ} / \mathrm{kg} / \mathrm{K}$. Comparando a equação acima com a (E.9), tem-se que $C_{V} \equiv C_{P}\left[1-P\left(\frac{\partial V}{\partial H}\right)_{P}\right]$ $16 \quad$ Note que se $V=V(H, P)$, então $\left(\frac{\partial V}{\partial H}\right)_{P}=\frac{V \alpha}{c_{p}}=\frac{1}{c_{p}}\left(\frac{\partial V}{\partial T}\right)_{P} ;$ e sendo gás ideal, então $V=\frac{n R T}{P}$, de
modo que $\left(\frac{\partial V}{\partial H}\right)_{P}=\frac{1}{c_{p}} \frac{n R}{P}$
$\quad$ Assim,

$$
\begin{aligned}
\left(\frac{\partial U}{\partial T}\right)_{P} & =n C_{p}\left[1-P\left(\frac{\partial V}{\partial H}\right)_{P}\right] \\
& =n C_{p}\left[1-P\left(\frac{1}{c_{p}} \frac{n R}{P}\right)\right] \\
& =n\left(C_{p}-R\right)
\end{aligned}
$$

e é restaurada a propriedade de gás ideal

$$
c_{p}=c_{v}+R
$$




\section{E.2 Entalpia}

O desvio ou flutuação relativa [17] é definida por

$$
\frac{\left\langle(F-\langle F\rangle)^{2}\right\rangle^{1 / 2}}{\langle F\rangle} \propto \frac{1}{\sqrt{n}} .
$$

onde $n$ é o número de partículas envolvidas na flutuação e $F$ é alguma propriedade termodinâmica do sistema.

No caso da entalpia, a flutuação relativa é dada por

$$
\frac{\left\langle(\Delta H)^{2}\right\rangle^{1 / 2}}{\langle H\rangle}=\lambda^{\prime} \frac{1}{\sqrt{n}}
$$

onde $\lambda(>0)$ é uma constante de proporcionalidade. Escrevendo $\langle H\rangle=m\langle h\rangle$, onde $\langle h\rangle$ é a entalpia específica média tem-se

$$
\left\langle(\Delta H)^{2}\right\rangle^{1 / 2}=\lambda^{\prime} \frac{m\langle h\rangle}{\sqrt{n}}
$$

Sabendo que a massa $m=n M_{0}$, onde $M_{0}$ é a massa molar, e assumindo $\langle h\rangle \cong h$, então a flutuação da entalpia é escrita como

$$
\left\langle(\Delta H)^{2}\right\rangle^{1 / 2}=\lambda \sqrt{n} h
$$

onde $\lambda=\lambda^{\prime} M_{0}$ e $h$ é entalpia específica.

\section{E.3 Limite Termodinâmico}

Qual é quantidade $n$ de moléculas que podem representar um elemento de volume? Ou melhor, qual é o tamanho mínimo do elemento de volume no qual é ainda válida a termodinâmica de equilíbrio. A resposta está no limite inferior da aplicabilidade da termodinâmica. O princípio da incerteza aplicado à termodinâmica [7] é dado por

$$
\Delta E \cdot \Delta\left(\frac{1}{T}\right) \gtrsim k_{B}
$$


a qual pode ser reescrita como

$$
\Delta U \cdot \frac{\Delta T}{T^{2}} \gtrsim k_{B}
$$

A variação da energia pode ser obtida com a equação (E.5), ou seja

$$
\left\langle(\Delta U)^{2}\right\rangle=n_{m} k_{B} T^{2} C_{V}
$$

onde $n_{m}$ é o número de moles e $C_{V}$ é a capacidade térmica molar. Assumindo que $\left\langle(\Delta U)^{2}\right\rangle^{1 / 2} \simeq$ $\Delta U$, então a incerteza pode ser escrita como

$$
T \sqrt{n_{m} k_{B} C_{V}} \cdot \frac{\Delta T}{T^{2}} \gtrsim k_{B}
$$

ou

$$
n_{m} C_{V} \cdot\left(\frac{\Delta T}{T}\right)^{2} \gtrsim k_{B}
$$

$\operatorname{mas} n_{m}=n / N_{A}$ de modo que resulta

$$
n \frac{C_{V}}{R} \cdot\left(\frac{\Delta T}{T}\right)^{2} \gtrsim 1
$$

ou

$$
n \gtrsim \frac{R}{\epsilon_{T}^{2} c_{v}}
$$

onde $R(=0.461526 \mathrm{~kJ} / \mathrm{kg} / \mathrm{K})$ e $c_{v}$ são específicos e $\epsilon_{T}=\Delta T / T$ é a incerteza da temperatura. Então, considerando o calor específico a pressão constante para o gás ideal e $\epsilon_{T} \sim 0.05$, ou seja $5 \%$ de incerteza, tem-se que $N \sim 100$. Portanto, admite-se válida a termodinâmica para o elemento de volume. 


\section{$\mathbf{F}$ \\ Experimentos com a Nucleação da Água}

\section{F.1 Sobre a experiência}

O experimento consta de uma câmara de expansão, conforme esquematizado na Figura F.32, onde é estudado o processo nucleação homogênea pelo método do pulso de nucleação [33][36]. Uma determinada quantidade de vapor de água e de gás carregador previamente misturados é transferida para a câmara de expansão (chamber volume), que se encontra a temperatura $T_{0}$. Em seguida as pressões nos respectivos volumes são ajustadas de modo que a subseqüente expansão leve sempre à mesma temperatura experimental, $T_{\exp }$. Com isso, estabelece-se uma pressão inicial, $P_{0}$, dentro da câmara de expansão. Depois de estabelecido o equilíbrio, o sistema é expandido adiabaticamente até a pressão $P_{\exp }$, resultando um estado metaestável, quando inicia-se a transição de fase. Para medir a taxa de nucleação, é feita uma recompressão ao se abrir a válvula de recompressão, diminuindo significativamente a formação de novos núcleos enquanto que os já formado continuam a crescer. A membrana de teflon funciona como um reservatório de volume, onde o objetivo destes volumes adicionais é suavizar o pulso de recompressão (diminuir a agitação mecânica). Possíveis turbulências são controladas com a placa com furos. As micros gotas formadas são contadas por meio de espalhamento de luz.

A pressão parcial do vapor $P_{v a p, 0}$ dentro da câmara, antes da expansão é determinada a partir da pressão total inicial $P_{0}$, por

$$
P_{v a p, 0}={ }_{96} \omega_{v} P_{0}
$$




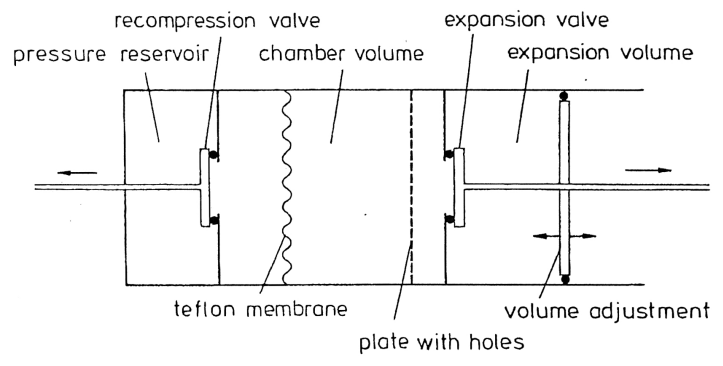

Fig. F.32- Esquema do equipamento usado no estudo da nucleação.

onde $\omega_{v}$ é a fração de vapor na mistura. A supersaturação $S_{s}$ é obtida através da pressão total inicial

$$
S_{s}=\frac{P_{v a p, 0}}{P_{e}\left(T_{\exp }\right)}
$$

onde $P_{e}\left(T_{\exp }\right)$ é a pressão de vapor no equilíbrio de fases e $T_{\exp }$ é a temperatura de nucleação.

Com a expansão adiabática do vapor de água até $P_{\text {exp }}$, inicia-se a nucleação. Este processo é muito rápido, de modo que uma recompressão do vapor (usualmente entre 2 e $6 \%$ da $\Delta P_{\max }$ ) é escolhida para reduzir a taxa de nucleação $J$ em pelo menos duas ordens de grandeza. $\mathrm{O}$ intervalo de tempo entre expansão e recompressão define um pulso de pressão, $\Delta t_{\exp } \cong 1 \mathrm{~ms}$, conforme Figura F.33. Com este procedimento o número de núcleos por unidade de volume ou a densidade de núcleos, $N_{\exp }$, tende a estabilizar-se (aproximação de estado estacionário). Os núcleos formados durante o pulso de nucleação podem ainda crescer até o tamanho de uma micro gota. A densidade de micro gotas $N_{\text {exp }}$ é observada por espalhamento de luz, de modo que a taxa de nucleação é dada por

$$
J=\frac{N_{\exp }}{\Delta t_{\exp }}
$$

admitindo o processo de nucleação estacionário, onde $\Delta t_{\exp }$ é o pulso de pressão.

Wölk-Strey conseguiram um bom controle da expansão seguida da recompressão, o que permitiu a reprodução do experimento com boa acurácia. A Figura F.32 mostra que o controle é obtido com o reservatório de pressão. 
A diferença média obtida sobre os pulsos, $\Delta P=P_{0}-P_{\text {exp }}$, e dividida por $P_{0}$ permite calcular a temperatura de nucleação $T_{\exp }$ de acordo com a lei de Poisson [33][36]

$$
T_{\exp }=T_{0}\left(\frac{P_{\exp }}{P_{0}}\right)^{\frac{\kappa-1}{\kappa}},
$$

onde $T_{0}$ é a temperatura inicial da câmara de expansão e $\kappa$ é a razão entre os calores específicos calculada através da fórmula de Richarz [33]

$$
\kappa=1+\left[\frac{\omega_{v}}{\kappa_{v}-1}+\frac{1-\omega_{v}}{\kappa_{g}-1}\right]^{-1}
$$

onde $\omega_{v}$ é a fração de vapor na mistura, $\kappa_{v}(=1.343)$ e $\kappa_{g}(=5 / 3)$ são as razões entre os calores específicos para o vapor e para o gás carregador, respectivamente.

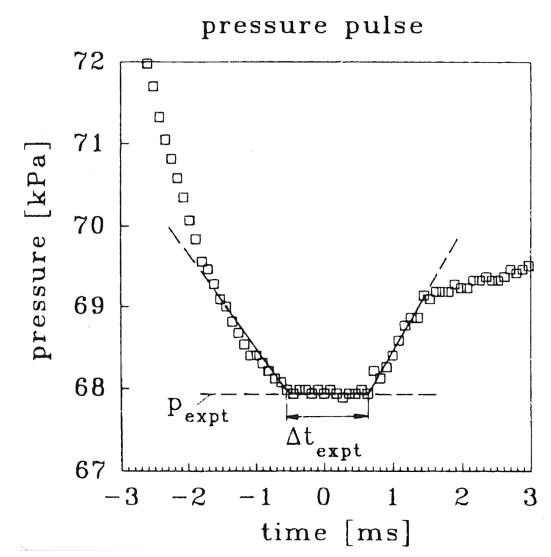

Fig. F.33- $\Delta t_{\exp }$ e $\Delta P_{\text {exp }}$ são encontrados via cálculo do trapézio.

Cabe notar que em geral gás carregador é um gás nobre como o Argônio. Wölk-Strey [36] mostraram que estes gases não interferem na nucleação da água. A Figura F.34 mostra como diferentes gases denominados carregadores afetam apenas a rapidez com que o sistema atinge a metaestabilidade. 


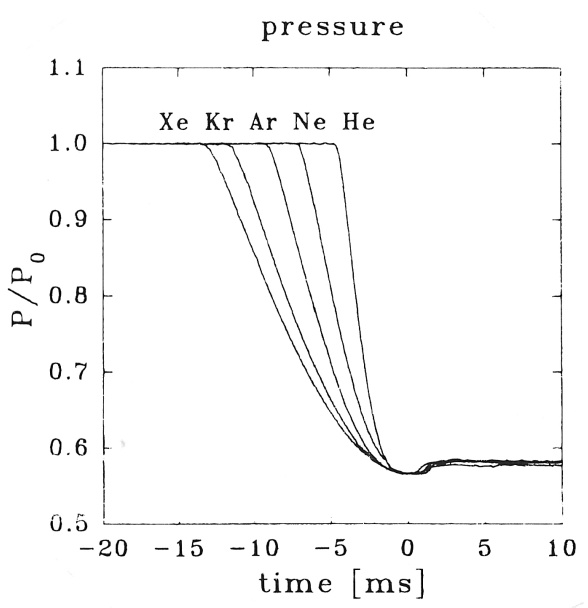

Fig. F.34- Os trabalhos de Strey et al. [33][36] mostram que o gás carregador não interfere na nucleação do vapor de água.

\section{F.2 Análise dos Dados}

Viisanen et al [33] e Wölk-Strey [36] realizaram uma série de experiências, organizadas pela temperatura do estado metaestável $\left(T_{\exp }\right)$ e pela quantidade de vapor de água na mistura gasosa. Os dados coletados por Wölk-Strey e organizados pela taxa de nucleação em função da supersaturação a temperatura constante estão na Figura F.35. Verifica-se claramente que os pontos experimentais encontram-se sobre uma reta.

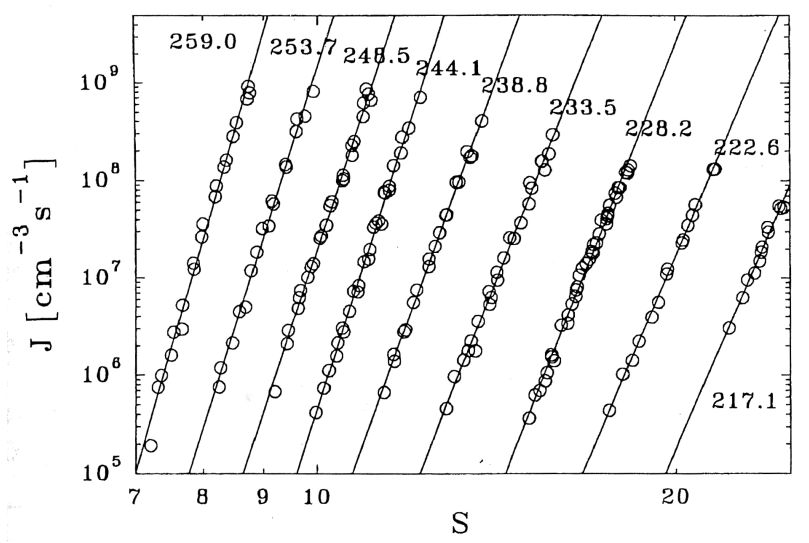

Fig. F.35- Independente do modelo clássico, $\partial \ln J / \partial \ln S_{s}=n^{*}$

Com a taxa de nucleação $J$ e a supersaturação $S_{s}$ calculou-se através do método dos mínimos quadrados, via sofware Maple $\mathrm{V}$, o núcleo crítico $n^{*}$ para cada série de experiências. 
O procedimento resulta uma reta

$$
\ln J=a+\left(n^{*}\right) \ln S_{s},
$$

referente a cada série, em que o coeficiente angular é o núcleo crítico, conforme sugerido pela equação (5.37)

$$
\left(\frac{\partial \ln J}{\partial \ln S_{s}}\right)_{T} \cong n^{*} .
$$

Para o presente trabalho, selecionou-se o ponto que apresentou o menor erro experimental. Em seguida, calculou-se novamente o tamanho do núcleo crítico para cada conjunto de experiências realizadas à mesma temperatura. Novamente escolheu-se o ponto que apresentou o menor erro experimental. Foi com os dados deste ponto experimental que se trabalhou nos cálculos de $n^{*}$ obtido pela TEN.

\section{F.2.1 Resultados: Viisanen et al.}

Seguem os resultados dos cálculos propostos acima para todas as séries de experiências feitas por Viisanen et al. [33]. Em cada uma delas observa-se os seguintes dados

$$
\begin{array}{ll}
T_{H 20, A r} & \text { temperatura inicial da mistura Água e Argônio } \\
T_{0} /\left({ }^{\circ} \mathrm{C}\right) & \text { temperatura inicial da câmara onde colocou-se a mistura } \mathrm{Ar}-\mathrm{H}_{2} \mathrm{O} \\
\omega_{v} & \text { fração do vapor de água na mistura } \\
P_{0} /(\mathrm{kPa}) & \text { pressão inicial total }\left(\mathrm{Ar}-\mathrm{H}_{2} \mathrm{O}\right) \\
\Delta P / P_{0} & =\left(P_{0}-P_{\text {exp }}\right) / P_{0}, \text { pulso de pressão } \\
J /\left(\mathrm{cm}^{-3} \mathrm{~s}^{-1}\right) & \text { taxa de nucleação experimental } \\
T / \mathrm{K} & \text { temperatura experimental no estado metaestável } \\
S_{s} & \text { supersaturação }
\end{array}
$$

Tabela G.7 - Série 1 - Mistura $\mathrm{H}_{2} \mathrm{O}$ e Argônio ou Xenônio a $T_{H 20, X e}=259 \mathrm{~K}$ e $T_{0}=33^{\circ} \mathrm{C}$

\begin{tabular}{|c|c|c|c|c|c|c|c|}
\hline Ensaio & $\omega_{v}$ & $P_{0} /(\mathrm{kPa})$ & {$\left[\Delta P / P_{0}\right]$} & $J /\left(\mathrm{cm}^{-3} \mathrm{~s}^{-1}\right)$ & $T /(\mathrm{K})$ & $S_{s}$ & $n_{\exp }^{*}$ \\
\hline$B$ & 0.02127 & 120.23 & 0.3451 & $6.80 \times 10^{7}$ & 258.99 & 8.19 & 41.48 \\
\hline
\end{tabular}

Tabela G.8 - Série 2 - Mistura $\mathrm{H}_{2} \mathrm{O}$ e Argônio ou Xenônio a $T_{H_{2} 0, A r}=253.7 \mathrm{~K}$ e $T_{0}=33^{\circ} \mathrm{C}$

\begin{tabular}{|c|c|c|c|c|c|c|c|}
\hline Ensaio & $\omega_{v}$ & $P_{0} /(\mathrm{kPa})$ & {$\left[\Delta P / P_{0}\right]$} & $J /\left(\mathrm{cm}^{-3} \mathrm{~s}^{-1}\right)$ & $T /(\mathrm{K})$ & $S_{s}$ & $n_{\exp }^{*}$ \\
\hline$C$ & 0.01610 & 122.67 & 0.3779 & $1.40 \times 10^{8}$ & 253.65 & 9.40 & 38.29 \\
\hline
\end{tabular}

Tabela G.9 - Série 3 - Mistura $\mathrm{H}_{2} \mathrm{O}$ e Argônio ou Xenônio a $T_{H_{2} 0, A r}=248.5 \mathrm{~K}$ e $T_{0}=33^{\circ} \mathrm{C}$ 


\begin{tabular}{|c|c|c|c|c|c|c|c|}
\hline Ensaio & $\omega_{v}$ & $P_{0} /(\mathrm{kPa})$ & {$\left[\Delta P / P_{0}\right]$} & $J /\left(\mathrm{cm}^{-3} \mathrm{~s}^{-1}\right)$ & $T /(\mathrm{K})$ & $S_{s}$ & $n_{\exp }^{*}$ \\
\hline$F$ & 0.01271 & 106.51 & 0.4086 & $4.90 \times 10^{6}$ & 248.5 & 9.63 & 36.52 \\
\hline
\end{tabular}

Tabela G.10 - Série 4 - Mistura $\mathrm{H}_{2} \mathrm{O}$ e Argônio ou Xenônio a $T_{H_{2} 0, A r}=244.1 \mathrm{~K}$ e $T_{0}=$ $15,63^{\circ} \mathrm{C}$

\begin{tabular}{|c|c|c|c|c|c|c|c|}
\hline Ensaio & $\omega_{v}$ & $P_{0} /(\mathrm{kPa})$ & {$\left[\Delta P / P_{0}\right]$} & $J /\left(\mathrm{cm}^{-3} \mathrm{~s}^{-1}\right)$ & $T /(\mathrm{K})$ & $S_{s}$ & $n_{\text {exp }}^{*}$ \\
\hline$H$ & 0.008022 & 120.23 & 0.3445 & $8.70 \times 10^{7}$ & 244.08 & 11.50 & 37.75 \\
\hline
\end{tabular}

Tabela G.11 - Série 5 - Mistura $\mathrm{H}_{2} \mathrm{O}$ e Argônio ou Kriptônio a $T_{H_{2} 0, K r}=238.8 \mathrm{~K}$ e $T_{0}=$ $15.63^{\circ} \mathrm{C}$

\begin{tabular}{|c|c|c|c|c|c|c|c|}
\hline Ensaio & $\omega_{v}$ & $P_{0} /(\mathrm{kPa})$ & {$\left[\Delta P / P_{0}\right]$} & $J /\left(\mathrm{cm}^{-3} \mathrm{~s}^{-1}\right)$ & $T /(\mathrm{K})$ & $S_{s}$ & $n_{\exp }^{*}$ \\
\hline$L$ & 0.005744 & 117.83 & 0.3791 & $2.10 \times 10^{7}$ & 238.81 & 12.57 & 33.32 \\
\hline
\end{tabular}

Tabela G.12 - Série 6 - Mistura $\mathrm{H}_{2} \mathrm{O}$ e Argônio ou Kriptônio a $T_{H_{2} 0, A r}=233.5 \mathrm{~K}$ e $T_{0}=$ $15.63^{\circ} \mathrm{C}$

\begin{tabular}{|c|c|c|c|c|c|c|c|}
\hline Ensaio & $\omega_{v}$ & $P_{0} /(\mathrm{kPa})$ & {$\left[\Delta P / P_{0}\right]$} & $J /\left(\mathrm{cm}^{-3} \mathrm{~s}^{-1}\right)$ & $T /(\mathrm{K})$ & $S_{s}$ & $n_{\exp }^{*}$ \\
\hline$N$ & 0.004206 & 106.50 & 0.4127 & $1.80 \times 10^{6}$ & 233.52 & 13.41 & 30.75 \\
\hline
\end{tabular}

Tabela G.13 - Série 7 - Mistura $\mathrm{H}_{2} \mathrm{O}$ e Hélio ou Neônio ou Argônio ou Kriptônio ou Xenônio a $T_{H_{2} 0, A r}=228 \mathrm{~K} \mathrm{e} T_{0}=15.63^{\circ} \mathrm{C}$

\begin{tabular}{|c|c|c|c|c|c|c|c|}
\hline Ensaio & $\omega_{v}$ & $P_{0} /(\mathrm{kPa})$ & {$\left[\Delta P / P_{0}\right]$} & $J /\left(\mathrm{cm}^{-3} \mathrm{~s}^{-1}\right)$ & $T /(\mathrm{K})$ & $S_{s}$ & $n_{\exp }^{*}$ \\
\hline$R$ & 0.002905 & 117.83 & 0.4455 & $1.90 \times 10^{7}$ & 228.19 & 17.01 & 28.07 \\
\hline
\end{tabular}

Tabela G.14 - Série 8 - Mistura $\mathrm{H}_{2} \mathrm{O}$ e Argônio a $T_{H_{2} 0, A r}=222.6 \mathrm{~K}$ e $T_{0}=15.63^{\circ} \mathrm{C}$

\begin{tabular}{|c|c|c|c|c|c|c|c|}
\hline Ensaio & $\omega_{v}$ & $P_{0} /(\mathrm{kPa})$ & {$\left[\Delta P / P_{0}\right]$} & $J /\left(\mathrm{cm}^{-3} \mathrm{~s}^{-1}\right)$ & $T /(\mathrm{K})$ & $S_{s}$ & $n_{\exp }^{*}$ \\
\hline$V$ & 0.002016 & 122.68 & 0.4786 & $1.30 \times 10^{8}$ & 222.63 & 21.51 & 28.25 \\
\hline
\end{tabular}

Tabela G.15 - Série 9 - Mistura $\mathrm{H}_{2} \mathrm{O}$ e Argônio a $T_{H_{2} 0, A r}=217.1 \mathrm{~K}$ e $T_{0}=15.63^{\circ} \mathrm{C}$

\begin{tabular}{|c|c|c|c|c|c|c|c|}
\hline Ensaio & $\omega_{v}$ & $P_{0} /(\mathrm{kPa})$ & {$\left[\Delta P / P_{0}\right]$} & $J /\left(\mathrm{cm}^{-3} \mathrm{~s}^{-1}\right)$ & $T /(\mathrm{K})$ & $S_{s}$ & $n_{\exp }^{*}$ \\
\hline$W$ & 0.001416 & 106.51 & 0.5101 & $1.90 \times 10^{7}$ & 217.12 & 23.64 & 27.79 \\
\hline
\end{tabular}




\section{F.2.2 Resultados: Wölk-Strey}

Segue os resultados dos cálculos propostos acima para todas as séries de experiências feitas por Strey et al. [36]

Tabela $G .16$ - Série A - Mistura $\mathrm{H}_{2} \mathrm{O}$ e Argônio a $T_{H 20, A r}=260 \mathrm{~K}$ e $T_{0}=30^{\circ} \mathrm{C}$

\begin{tabular}{|c|c|c|c|c|c|c|c|}
\hline Ensaio & $\omega_{v}$ & $P_{0} /(\mathrm{kPa})$ & {$\left[\Delta P / P_{0}\right]$} & $J /\left(\mathrm{cm}^{-3} \mathrm{~s}^{-1}\right)$ & $T /(\mathrm{K})$ & $S_{s}$ & $n_{\exp }^{*}$ \\
\hline A3 & 0.02623 & 92.88 & 0.3240 & $5.30 \times 10^{7}$ & 259.83 & 7.52 & 33.28 \\
\hline
\end{tabular}

Tabela G.17 - Série B - Mistura $\mathrm{H}_{2} \mathrm{O}$ e Argônio a $T_{H 20, A r}=250 \mathrm{~K}$ e $T_{0}=25^{\circ} \mathrm{C}$

\begin{tabular}{|c|c|c|c|c|c|c|c|}
\hline Ensaio & $\omega_{v}$ & $P_{0} /(\mathrm{kPa})$ & {$\left[\Delta P / P_{0}\right]$} & $J /\left(\mathrm{cm}^{-3} \mathrm{~s}^{-1}\right)$ & $T /(\mathrm{K})$ & $S_{s}$ & $n_{\exp }^{*}$ \\
\hline 1 & 0.01677 & 91.11 & 0.3601 & $2.42 \times 10^{9}$ & 249.84 & 10.45 & 32.52 \\
\hline
\end{tabular}

Tabela G.18 - Série C - Mistura $\mathrm{H}_{2} \mathrm{O}$ e Argônio a $T_{H 20, A r}=240 \mathrm{~K}$ e $T_{0}=20^{\circ} \mathrm{C}$

\begin{tabular}{|c|c|c|c|c|c|c|c|}
\hline Ensaio & $\omega_{v}$ & $P_{0} /(\mathrm{kPa})$ & {$\left[\Delta P / P_{0}\right]$} & $J /\left(\mathrm{cm}^{-3} \mathrm{~s}^{-1}\right)$ & $T /(\mathrm{K})$ & $S_{s}$ & $n_{\exp }^{*}$ \\
\hline 1 & 0.006797 & 94.48 & 0.3971 & $3.74 \times 10^{6}$ & 239.63 & 10.71 & 30.03 \\
\hline
\end{tabular}

Tabela G.19 - Série D - Mistura $\mathrm{H}_{2} \mathrm{O}$ e Argônio a $T_{H 20, A r}=230 \mathrm{~K}$ e $T_{0}=10^{\circ} \mathrm{C}$

\begin{tabular}{|c|c|c|c|c|c|c|c|}
\hline Ensaio & $\omega_{v}$ & $P_{0} /(\mathrm{kPa})$ & {$\left[\Delta P / P_{0}\right]$} & $J /\left(\mathrm{cm}^{-3} \mathrm{~s}^{-1}\right)$ & $T /(\mathrm{K})$ & $S_{s}$ & $n_{\exp }^{*}$ \\
\hline 1 & 0.003404 & 103.07 & 0.4087 & $1.91 \times 10^{8}$ & 229.58 & 16.06 & 25.75 \\
\hline
\end{tabular}

Tabela G.20 - Série E - Mistura $\mathrm{H}_{2} \mathrm{O}$ e Argônio a $T_{H 20, A r}=220 \mathrm{~K}$ e $T_{0}=10^{\circ} \mathrm{C}$

\begin{tabular}{|c|c|c|c|c|c|c|c|}
\hline Ensaio & $\omega_{v}$ & $P_{0} /(\mathrm{kPa})$ & {$\left[\Delta P / P_{0}\right]$} & $J /\left(\mathrm{cm}^{-3} \mathrm{~s}^{-1}\right)$ & $T /(\mathrm{K})$ & $S_{s}$ & $n_{\text {exp }}^{*}$ \\
\hline 4 & 0.001708 & 79.99 & 0.4744 & $5.61 \times 10^{6}$ & 218.98 & 18.57 & 20.73 \\
\hline
\end{tabular}




\section{Referências}

[1] ARFKEN, G.B.;WEBER, H.J. Mathematical Methods for Physicists. 4th ed. London: Academic Press, 1995. p. 319.

[2] CHUKANOV, VN; SKRIPOV, VP. Specific volumes of severely superheated water. High Temperature, v. 9, n. 4, p.672, 1971.

[3] DeHOFF, ROBERT T. Thermodynamics in Materials Science. Singapore: McGraw-Hill. 1993.

[4] DILlMANN, A.; MEIER, G. E. A. Homogeneous Nucleation of Supersaturated Water. Chem. Phys. Lett. v. 160, n.71, 1989.

[5] DillmanN, A.; MEIER, G. E. A. A Refined Droplet Approach to the Problem of Homogeneous Nucleation from the Vapor Phase. J. Chem. Phys. v. 94, n. 5, 1991.

[6] EVSTEFEEF , V. N.; CHUKANOV, V. N.; SKRIPOV, V. P. Specific volumes of superheated water. High Temperature, v. 15, n. 3, p. 550-552, 1977.

[7] FESHBACH, H. Small systems: when does thermodynamic apply?. Phys. Today. v. 9, Nov 1987.

[8] FINKBEINER, DANIEL T. Introdução às Matrizes e Transformações Lineares, Rio de Janeiro: Ao Livro Técnico, 1970. Cap. 5

[9] FRANKS, FELIX. Water, London: Royal Society of Chemistry. Paperbacks, 1984.

[10] GLANSDORFF, P.; PRIGOGINE, I. Thermodynamic Theory of Structure, Stability and Fluctuations, London: Wiley, 1974.

[11] GOODSTEIN, DAVID L. States of matter, New Jersey: Prentice-Hall, 1975. Chap. 4.

[12] GUTZOW, I.; SCHMELZER, J. The Vitreous State, Germany: Springer, 1995.

[13] KASHCHIEV, D. Nucleation: basic theory with applications, Oxford: ButterworthHeinemann. 2000.

[14] KESTIN, JOSEPH. A Course in Thermodynamics, v. 1 e 2. Massachusets: Blaisdell, 1966.

[15] KOPER, GER J. M.; REISS, HOWARD. Length Scale for the Constant Pressure Ensemble: Applications tp Small systems and Relation to Einstein Fluctuation Theory. J. Phys. Chem. v. 100, n. 1, p. 422-432, 1996. 
[16] LANDAU, L.; AJIEZER, A.; LIFSHITZ, E. Curso de Fisica General. Moscù: Mir. 1979.

[17] LANDAU, L. D.; LIFSHITZ, E. M. Statistical Physics. 3rd ed. Part 1. Great Britain: Butterworth-Heinemann. 1997. p. 333.

[18] MONTROLL, E. W.; LEBOWITZ, J. L. (eds.) Studies in Statistical Mechanics: Fluctuation Phenomena. v. VII. Amsterdan: North-Holland Publishing. 1979. Chapter 5: Penrose, O.; Lebowitz, J. L. Towards a rigorous molecular theory of metastability.

[19] MOKROSS, B. J. Nucleation Theory and Application. [in Nucleation Theory and Applications. J. W. P. Schmelzer, G. Röpke, V. B. Priezzhev (eds.). Russia. Joint Institute for Nuclear Research (JINR)] 1999.

[20] MOKROSS, B. J. Entropic Nucleation Theory. J. Non-Crys. Sol., v. 284, n. 91, 2001.

[21] MOKROSS, B. J. Nucleation Theory and Small System Thermodynamics. Mater. Phys. Mech., v. 6, p. 13-20, 2003.

[22] MOKROSS, B. J. e PASQUA, N. H. Comment on "Length Scale for the Constant Pressure Ensemble: Application to Small Systems and Relation to Einstein Fluctuation Theory" by Ger J. M. Koper and Howard Reiss, J. Phys. Chem. 100, 422-432, 1996. [Artigo submetido para J. Phys. Chem. em Jul/2007]

[23] PASQUA, NORBERTO HELIL. Sobre o Fenômeno da Nucleação, Dissertação de Mestrado, São Carlos: USP/IFSC. 2003.

[24] PASQUA, NORBERTO HELIL e MOKROSS, BERNHARD JOACHIM. Fluctuation Theory Applied to Water Vapor Nucleation. [Artigo aceito em: Schmelzer, J. W. P.; Röpke, G.; Priezzhev, V. B. (eds.) Nucleation Theory and Applications. Dubna: Joint Institute for Nuclear Research (JINR). Abr/2007]

[25] PENG, D.-Y.; ROBINSON, D. B. A new two-constant equation of state. Ind.Eng.Chem.Fundam., v. 15, n. 59, 1976.

[26] PRESS, WILliam H. et al. Numerical Recipes in Fortran: The Art of Scientific Computing, $2^{\text {nd }}$ ed., Cambridge: Univerty Press, 1992. Chap. 2.

[27] PRIGOGINE, I.; KONDEPUDI, D. Termodinâmica: dos Motores Térmicos às Estruturas Dissipativas, Lisboa: Odile Jacob, 1999.

[28] REISS, HOWARD. Methods of Thermodynamics, Minealona: Dover, 1996.

[29] REISS, H.; KEGEL; W. K.; KATZ, J. L. Resolution of the Problems of Replacement Free Energy, $1 / S_{s}$, and Internal Consistency in Nucleation Theory by Consideration of the Lenght Scale for Mixing Entropy. Phys. Rev. Lett, v. 78, n. 4506, 1997.

[30] SALINAS, S. R. A. Introdução à Física Estatítica, $2^{a}$ ed., São Paulo: Edusp, 1999. 
[31] SMITH, J. M.; NESS, H. C. Y.; ABBOTT, M. M. Introdução à Termodinâmica da Engenharia Química, $5^{a}$ ed., Rio de Janeiro: LTC. 2000. Apêndice F.

[32] TESTER, J.W.; MODELL, M. Thermodynamics and Its Applications, $3^{\text {rd }}$ ed., New Jersey: Prentice Hall. 1996.

[33] VIISANEN, Y.; STREY, R. ; REISS, H. Homogeneous nucletion rates for water. J. Chem. Phys, v. 99, p. 4680, 1993.

[34] WAGNER, W. et al. The IAPWS Industrial Formulation 1997 for the Thermodynamic Properties of Water and Steam. Journal of Engineering for Gas Turbines and Power. v. 122, January 2000.

[35] WAGNER, W.; PRUß, A. The IAPWS Formulation 1995 for the Thermodynamic Properties of Ordinary Water Substance for General and Scientific Use. J. Phys. Chem. Ref. Data, v. 31, n. 2, 2002.

[36] WÖLK, J.; STREY, R. Homogeneous Nucleation of $\mathrm{H}_{2} \mathrm{O}$ and $\mathrm{D}_{2} \mathrm{O}$ in Comparison: The Isotope Effect. J. Phys. Chem. B, v. 105, p. 11683, 2001.

[37] ZEMANSKY, M. W. Heat and Thermodynamics, $5^{a}$ ed., Tokio: McGraw-Hill, 1968. 